\title{
Iron-Catalyzed Silylation of (Hetero)Aryl Chlorides with $\mathrm{Et}_{3}$ SiBpin
}

Jia Jia, ${ }^{1, \#}$ Xiaoqin Zeng, ${ }^{2, \#}$ Zhengli Liu, ${ }^{2}$ Liang Zhao, ${ }^{1}$ Chun-Yang He, ${ }^{1 *}$ Xiao-Fei $\mathrm{Li}^{1 *}$ and Zhang Feng ${ }^{2,3 *}$

${ }^{1}$ Key Laboratory of Biocatalysis \& Chiral Drug Synthesis of Guizhou Province, Generic Drug Research Center of Guizhou Province, School of Pharmacy, Zunyi Medical University, Zunyi, Guizhou, 563003, P.R. China

${ }^{2}$ Chongqing Key Laboratory of Natural Product Synthesis and Drug Research, School of Pharmaceutical Sciences, Chongqing University, Chongqing 401331, P. R. China

3 Sichuan Key Laboratory of Medical Imaging \& Department of Chemistry, School of Preclinical Medicine, North Sichuan Medical College, Nanchong, Sichuan 63700o, P. R. China

\section{List of Contents}

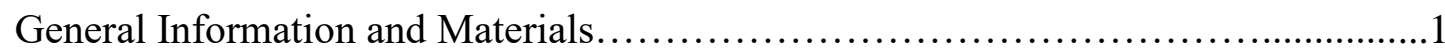

Optimization of Iron-Catalyzed Silylation of 4-biphenylyl chloride...................2

Optimization of Iron-Catalyzed Silylation of Aryl Chlorides...........................6

Mechanistic Studies............................................................ 10

General Procedure for the iron-catalyzed silylation of aryl chlorides............... 10

Experimental Procedures and Characterization Data for Products....................12

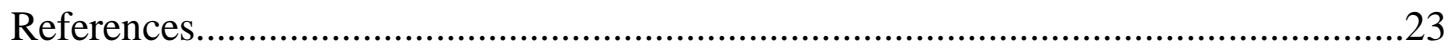

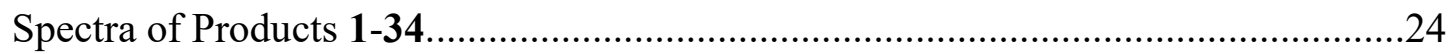


General information: ${ }^{1} \mathrm{H}$ NMR and ${ }^{13} \mathrm{C}$ NMR spectra were recorded on Agilent 400MR DD2 (400 MHz) or 600MR DD2 (600 MHz) spectrometer at ambient temperature. Chemical shifts $(\delta)$ are reported in ppm, and coupling constants $(J)$ are in Hertz $(\mathrm{Hz})$. The following abbreviations were used to explain the multiplicities: $\mathrm{s}=$ singlet, $\mathrm{d}=$ doublet, $\mathrm{t}=$ triplet, $\mathrm{q}=$ quartet, $\mathrm{m}=$ multiplet, sept $=$ septet. $\mathrm{NMR}$ yield was determined by ${ }^{1} \mathrm{H}$ NMR using mesitylene as an internal standard before working up the reaction.

Materials: All reagents that used were from commercial sources, unless otherwise specified. $\mathrm{FeI}_{2}(97 \%)$ was purchased from Alfa Aesar. $t$-BuONa (99\%) was purchased from Adamas. Diglyme and DMF were distilled under reduced pressure from $\mathrm{CaH}_{2}$. 1,4-Dioxane, THF, MTBE, $(i-\mathrm{Pr})_{2} \mathrm{O}$ and toluene were distilled from sodium and benzophenone immediately before used. 
Optimization of Iron-Catalyzed Silylation of 4-biphenylyl chloride (Table S1S6):

Table S1. Screening of Iron Sources and Ligand. ${ }^{a}$

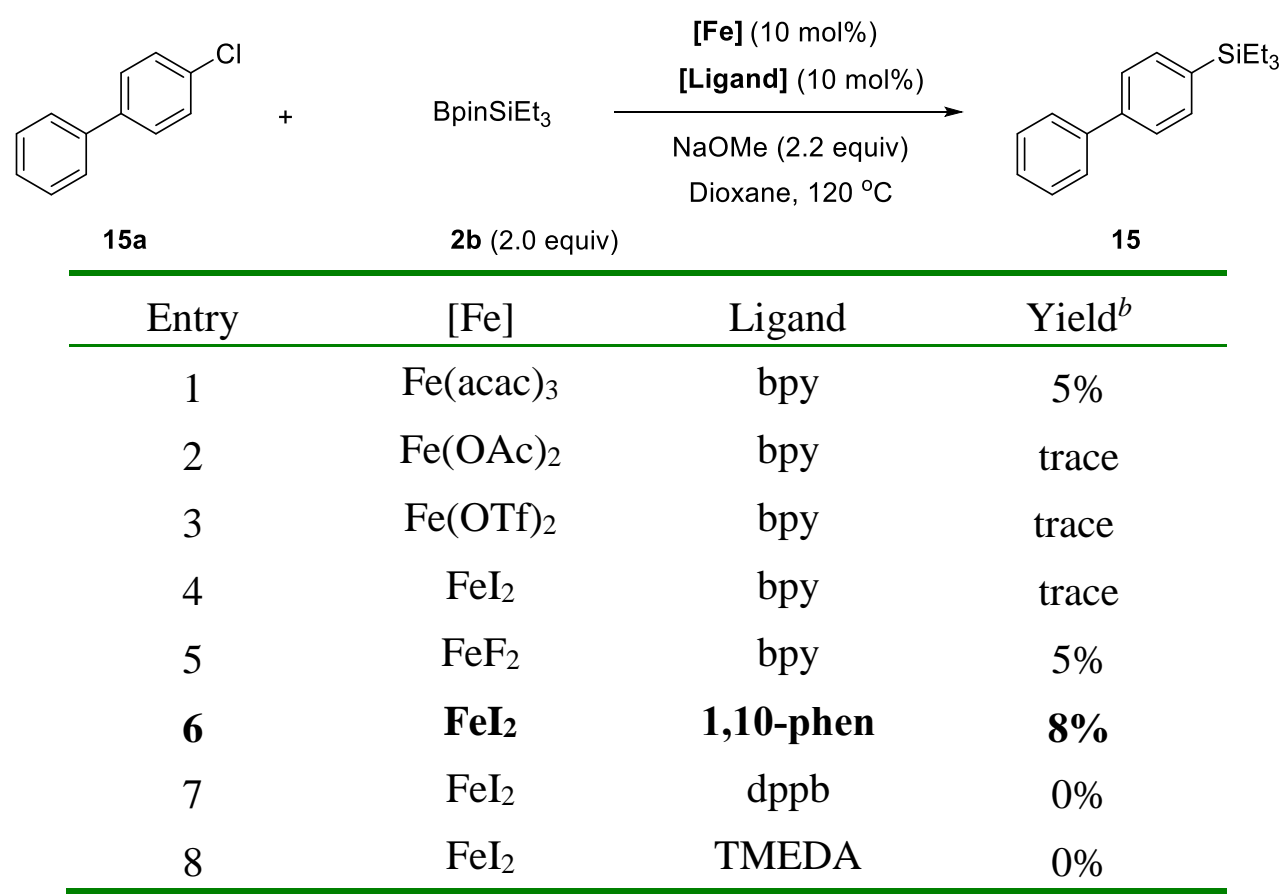

${ }^{a}$ Reaction conditions (unless otherwise specified): $\mathbf{1 5 a}(0.2 \mathrm{mmol}, 1.0$ equiv), $\mathbf{2 b}$ (2.0 equiv), $\mathrm{NaOMe}$ (2.2 equiv), [Fe] (10 mol\%), ligand $(10 \mathrm{~mol} \%)$, dioxane $(1.5 \mathrm{~mL}), 120^{\circ} \mathrm{C}, 12 \mathrm{~h}$, under an argon atmosphere. ${ }^{b}$ Determined by ${ }^{1} \mathrm{H}$ NMR using mesitylene as an internal standard.

Table S2. Screening of Bases. ${ }^{a}$

\begin{tabular}{|c|c|c|c|c|}
\hline \multirow{11}{*}{ 15a } & \multicolumn{3}{|c|}{$\begin{array}{l}\mathrm{Fel}_{2}(10 \mathrm{~mol} \%) \\
1,10 \text {-phen }(10 \mathrm{~mol} \%)\end{array}$} & \multirow{11}{*}{15} \\
\hline & Entry & Base & Yield $^{b}$ & \\
\hline & 1 & $\mathrm{~K}_{3} \mathrm{PO}_{4}$ & $0 \%$ & \\
\hline & 2 & KOAc & $0 \%$ & \\
\hline & 3 & $\mathrm{KF}$ & $0 \%$ & \\
\hline & 4 & $\mathrm{Cs}_{2} \mathrm{CO}_{3}$ & $0 \%$ & \\
\hline & 5 & $\mathrm{Et}_{3} \mathrm{~N}$ & $0 \%$ & \\
\hline & 6 & $t$-BuOK & trace & \\
\hline & 7 & $t$-BuOLi & $0 \%$ & \\
\hline & 8 & $t$-BuONa & $12 \%$ & \\
\hline & 9 & $t-\mathrm{BuOMg}$ & $0 \%$ & \\
\hline
\end{tabular}




$\begin{array}{ccc}10 & \text { NaOMe } & 8 \% \\ 11 & \text { KOMe } & \text { trace } \\ 12 & \text { LiOMe } & 0 \%\end{array}$

${ }^{a}$ Reaction conditions (unless otherwise specified): 15a ( $0.2 \mathrm{mmol}, 1.0$ equiv), $\mathbf{2} \mathbf{b}$ (2.0 equiv), base (2.2 equiv), $\mathrm{FeI}_{2}(10 \mathrm{~mol} \%), 1,10$-phen $(10 \mathrm{~mol} \%)$, dioxane $(1.5 \mathrm{~mL}), 120{ }^{\circ} \mathrm{C}, 12 \mathrm{~h}$, under an argon atmosphere. ${ }^{b}$ Determined by ${ }^{1} \mathrm{H}$ NMR using mesitylene as an internal standard.

\section{Table S3. Screening of Solvents. ${ }^{a}$}

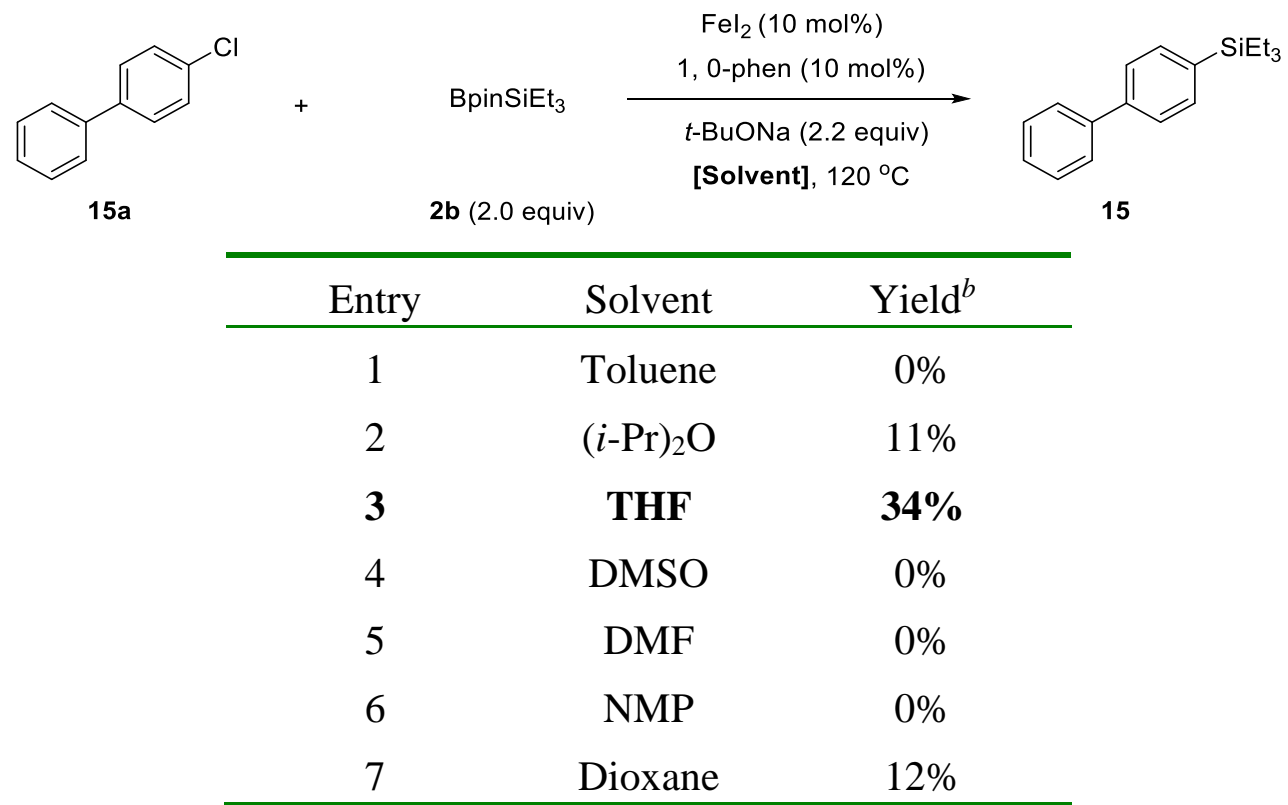

${ }^{a}$ Reaction conditions (unless otherwise specified): 15a ( $0.2 \mathrm{mmol}, 1.0$ equiv), $2 \mathbf{b}$ (2.0 equiv), $t$ BuONa (2.2 equiv), $\mathrm{FeI}_{2}(10 \mathrm{~mol} \%), 1,10$-phen $(10 \mathrm{~mol} \%)$, Solvent $(1.5 \mathrm{~mL}), 120^{\circ} \mathrm{C}, 12 \mathrm{~h}$, under an argon atmosphere. ${ }^{b}$ Determined by ${ }^{1} \mathrm{H}$ NMR using mesitylene as an internal standard.

Table S4. Screening of Iron Sources. ${ }^{a}$

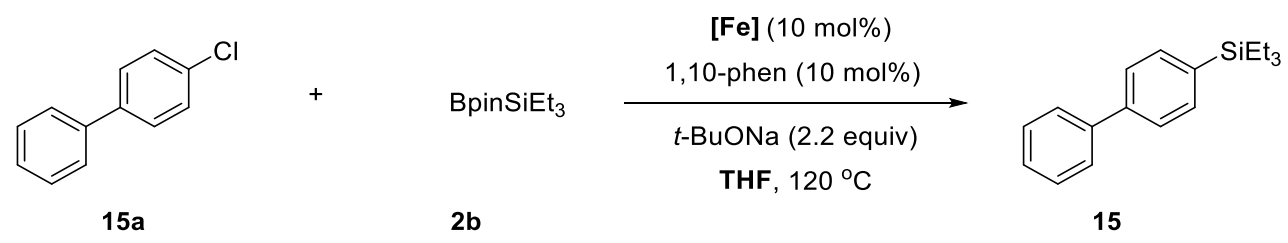

\begin{tabular}{ccc}
\hline Entry & {$[\mathrm{Fe}]$} & Yield $^{b}$ \\
\hline 1 & {$\left[\mathrm{CH}_{3}\left(\mathrm{CH}_{2}\right)_{16} \mathrm{COD}\right]_{3} \mathrm{Fe}$} & trace \\
2 & $\mathrm{Fe}(\mathrm{OAc})_{2}$ & $16 \%$ \\
3 & $\mathrm{FeBr}_{2}$ & $26 \%$ \\
4 & $\mathrm{Fe}(\mathrm{OTs})_{3}$ & $14 \%$ \\
5 & Tris(dibenzoylMethan & $23 \%$ \\
& ato) Iron (III) &
\end{tabular}


${ }^{a}$ Reaction conditions (unless otherwise specified): $\mathbf{1 5 a}(0.2 \mathrm{mmol}, 1.0$ equiv), $\mathbf{2 b}$ (2.0 equiv), $t$ BuONa (2.2 equiv), [Fe] (10 mol\%), 1, 10-phen (10 mol\%), THF $(1.5 \mathrm{~mL}), 120^{\circ} \mathrm{C}, 12 \mathrm{~h}$, under an argon atmosphere. ${ }^{b}$ Determined by ${ }^{1} \mathrm{H}$ NMR using mesitylene as an internal standard.

Table S5. Screening of Ligands. ${ }^{a}$

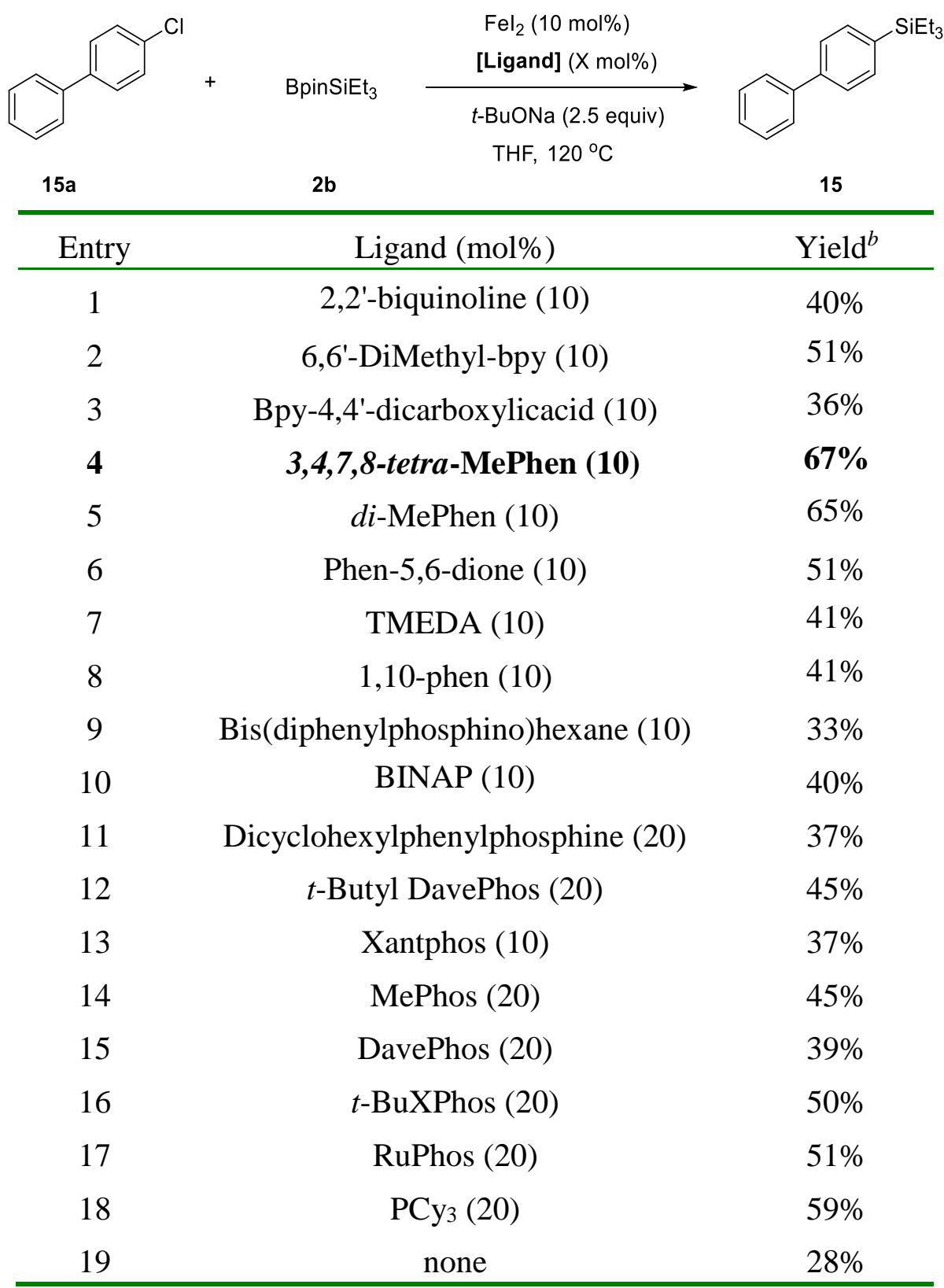

${ }^{a}$ Reaction conditions (unless otherwise specified): 19 a $(0.2 \mathrm{mmol}, 1.0$ equiv), 2a (3.0 equiv), $t$ BuONa (2.5 equiv), $\mathrm{FeI}_{2}(10 \mathrm{~mol} \%)$, Ligand, THF $(1.5 \mathrm{~mL}), 120{ }^{\circ} \mathrm{C}, 12 \mathrm{~h}$, under an argon atmosphere. ${ }^{b}$ Determined by ${ }^{1} \mathrm{H}$ NMR using mesitylene as an internal standard. 
Table S6. Optimization of Iron-Catalyzed Silylation of 4-biphenylyl chloride. ${ }^{a}$
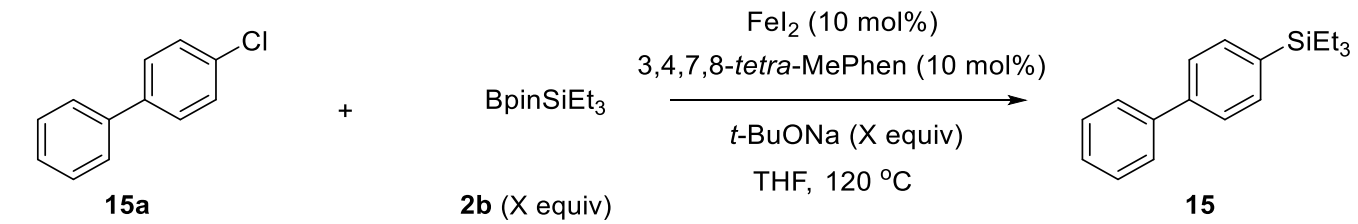

\begin{tabular}{ccccc}
\hline Entry & $\mathbf{2 b}$ (equiv) & $t$-BuONa (equiv) & THF (mL) & Yield $^{b}$ \\
\hline 1 & 3.0 & 2.5 & 1.5 & $67 \%$ \\
2 & 3.0 & 3.0 & 1.5 & $53 \%$ \\
3 & 3.0 & 3.5 & 1.5 & $30 \%$ \\
4 & 3.5 & 2.5 & 1.5 & $70 \%$ \\
5 & 3.5 & 2.5 & 2.0 & $56 \%$ \\
6 & 3.5 & 3.0 & 1.5 & $61 \%$ \\
7 & 3.5 & 3.5 & 1.5 & $45 \%$ \\
$\mathbf{8}$ & 3.5 & 4.0 & 1.5 & $41 \%$ \\
9 & $\mathbf{3 . 7}$ & $\mathbf{2 . 5}$ & 1.5 & $\mathbf{8 4 \%}(\mathbf{7 6 \%})$ \\
10 & 4.0 & 2.5 & 1.5 & $\mathbf{7 7 \%}$ \\
11 & 4.0 & 2.5 & 2.0 & $57 \%$ \\
12 & 4.0 & 3.0 & 1.5 & $63 \%$ \\
13 & 4.0 & 3.5 & 1.5 & $64 \%$ \\
14 & 4.0 & 4.0 & 1.5 & $50 \%$ \\
$15^{c}$ & $\mathbf{3 . 7}$ & $\mathbf{2 . 5}$ & 1.5 & $\mathbf{9 2 \%}(\mathbf{9 0 \%})$ \\
\hline
\end{tabular}

${ }^{a}$ Reaction conditions (unless otherwise specified): $\mathbf{1 5 a}$ (0.2 mmol, 1.0 equiv), $\mathbf{2 b}, t$-BuONa, $\mathrm{FeI}_{2}$ (10 mol\%), tetra-MePhen (10 mol\%), THF, $120^{\circ} \mathrm{C}, 12 \mathrm{~h}$, under an argon atmosphere. ${ }^{b}$ Determined by ${ }^{1} \mathrm{H}$ NMR using mesitylene as an internal standard. ${ }^{c}$ This reaction was conducted at $120^{\circ} \mathrm{C}$

Optimization of Iron-Catalyzed Silylation of Aryl Chlorine 1a (Table S7-S13):

Table S7. Screening of Ligands. ${ }^{a}$

\begin{tabular}{|c|c|c|c|}
\hline $1 \mathrm{a}$ & $\begin{array}{c}\text { BpinSiEt }_{3} \\
\text { 2b (3.5 equiv) }\end{array}$ & $\begin{array}{l}\mathrm{Fel}_{2}(10 \mathrm{~mol} \%) \\
\text { [Ligand] }(\mathrm{X} \text { mol\%) } \\
t \text {-BuONa (2.5 equiv) } \\
\mathrm{THF}, 120^{\circ} \mathrm{C}\end{array}$ & 1 \\
\hline Entry & \multicolumn{2}{|c|}{ Ligand (mol\%) } & Yield $^{b}$ \\
\hline 1 & \multicolumn{2}{|c|}{ DiMe-bpy (10) } & $35 \%$ \\
\hline 2 & \multicolumn{2}{|c|}{ 2, 2'-bipyridine-4, 4'-dicarboxylic acid (10) } & $22 \%$ \\
\hline 3 & \multicolumn{2}{|c|}{ Neocuproine (10) } & $47 \%$ \\
\hline 4 & \multicolumn{2}{|c|}{ 3,4,7,8-tetra-MePhen (10) } & $65 \%$ \\
\hline
\end{tabular}


${ }^{a}$ Reaction conditions (unless otherwise specified): $1 \mathbf{1 a}(0.2 \mathrm{mmol}, 1.0$ equiv), $\mathbf{2 b}$ (3.5 equiv), $t$ BuONa (2.5 equiv), $\mathrm{FeI}_{2}(10 \mathrm{~mol} \%)$, Ligand, THF $(1.5 \mathrm{~mL}), 120{ }^{\circ} \mathrm{C}, 12 \mathrm{~h}$, under an argon atmosphere. ${ }^{b}$ Determined by ${ }^{1} \mathrm{H}$ NMR using mesitylene as an internal standard.

\section{Table S8. Screening of Iron Sources and Bases. ${ }^{a}$}
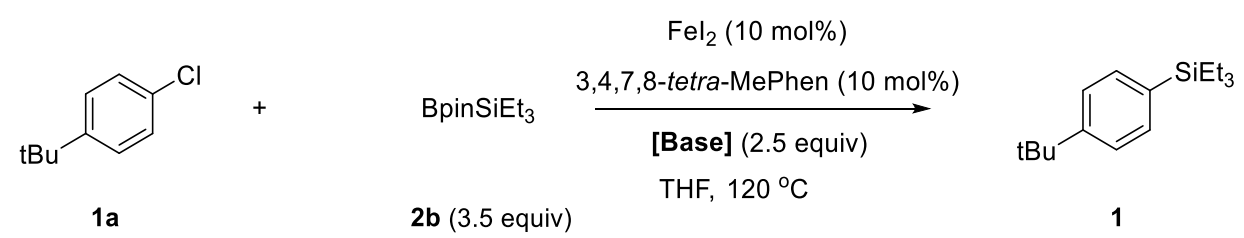

\begin{tabular}{ccc}
\hline Entry & Base & Yield $^{b}$ \\
\hline 1 & $\mathrm{Et}_{3} \mathrm{~N}$ & $0 \%$ \\
2 & $\mathrm{TMEDA}$ & $0 \%$ \\
3 & $\mathrm{Cs}_{2} \mathrm{CO}_{3}$ & $0 \%$ \\
4 & $\boldsymbol{t}$-BuONa & $\mathbf{6 5 \%}$ \\
5 & none & $0 \%$ \\
\hline
\end{tabular}

${ }^{a}$ Reaction conditions (unless otherwise specified): 1a (0.2 mmol, 1.0 equiv), $\mathbf{2} \mathbf{b}$ (3.5 equiv), Base (2.5 equiv), $\mathrm{FeI}_{2}(10 \mathrm{~mol} \%), 3,4,7,8$-tetra-MePhen $(10 \mathrm{~mol} \%)$, THF $(1.5 \mathrm{~mL}), 120^{\circ} \mathrm{C}, 12 \mathrm{~h}$, under an argon atmosphere. ${ }^{b}$ Determined by ${ }^{1} \mathrm{H}$ NMR using mesitylene as an internal standard.

Table S9. Screening of Solvents. ${ }^{a}$

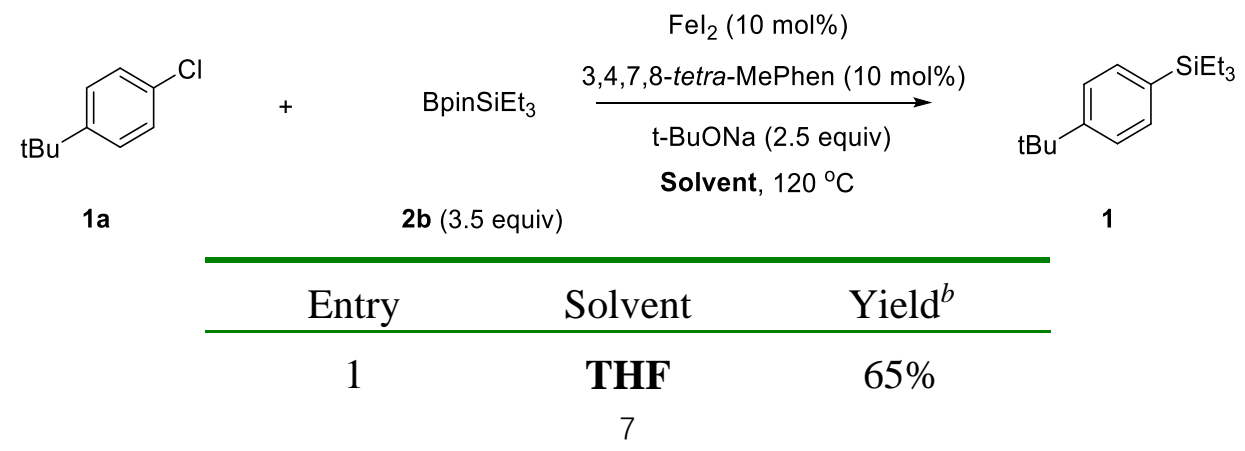




\begin{tabular}{ccc}
2 & DMF & $0 \%$ \\
3 & DME & $0 \%$ \\
4 & DMA & $0 \%$ \\
5 & MeCN & $0 \%$ \\
6 & NMP & $0 \%$ \\
7 & DMSO & $0 \%$ \\
\hline
\end{tabular}

${ }^{a}$ Reaction conditions (unless otherwise specified): 1a (0.2 mmol, 1.0 equiv), $2 \mathbf{b}$ (3.5 equiv), $t$ BuONa (2.5 equiv), $\mathrm{FeI}_{2}$ (10 mol\%), 3,4,7,8-tetra-MePhen (10 mol\%), Solvent (1.5 mL), $120{ }^{\circ} \mathrm{C}$, $12 \mathrm{~h}$, under an argon atmosphere. ${ }^{b}$ Determined by ${ }^{1} \mathrm{H}$ NMR using mesitylene as an internal standard.

Table S10. Screening of Iron Sources. ${ }^{a}$

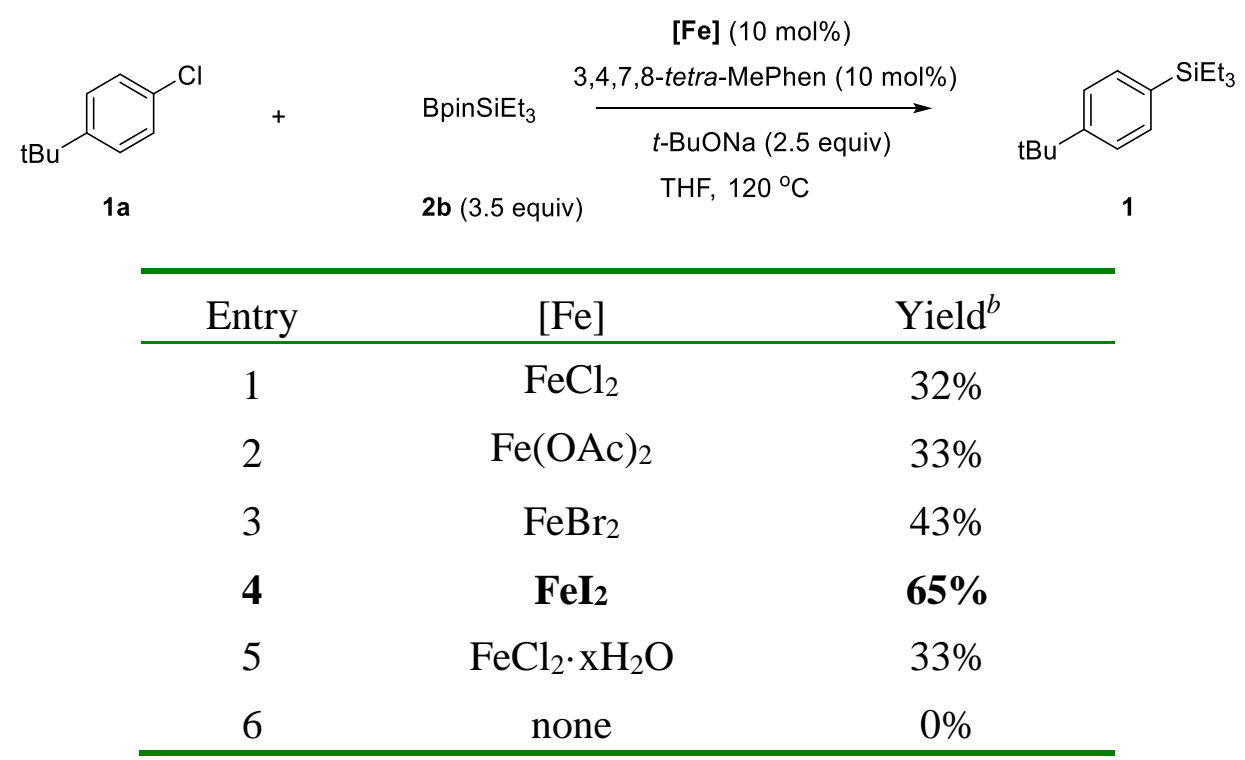

${ }^{a}$ Reaction conditions (unless otherwise specified): $1 \mathbf{a}(0.2 \mathrm{mmol}, 1.0$ equiv), $2 \mathbf{b}$ (3.5 equiv), $t$ BuONa (2.5 equiv), [Fe] (10 mol\%), 3,4,7,8-tetra-MePhen (10 mol\%), THF $(1.5 \mathrm{~mL}), 120^{\circ} \mathrm{C}, 12 \mathrm{~h}$, under an argon atmosphere. ${ }^{b}$ Determined by ${ }^{1} \mathrm{H}$ NMR using mesitylene as an internal standard.

Table S11. Optimization of Iron-Catalyzed Silylation of 1a. ${ }^{a}$

\begin{tabular}{|c|c|c|c|c|}
\hline & $a$ & $\begin{array}{l}\mathrm{BpinSiEt}_{3} \\
\text { 2b (X equiv) }\end{array}$ & $\begin{array}{l}\mathrm{Fel}_{2}(10 \mathrm{~mol} \%) \\
\text { 8-tetra-MePhen }(10 \mathrm{~mol} \%) \\
\underset{t \text {-BuONa }(\mathrm{X} \text { equiv })}{\longrightarrow} \\
\mathrm{THF}, \mathrm{X}^{\circ} \mathrm{C}\end{array}$ & 1 \\
\hline Entry & $\mathbf{2 b}$ (equiv) & $t$-BuONa (equiv) & Temp $\left({ }^{\circ} \mathrm{C}\right)$ & Yield $^{b}$ \\
\hline 1 & 3.0 & 2.5 & 120 & $33 \%$ \\
\hline 2 & 3.5 & 2.5 & 120 & $65 \%$ \\
\hline 3 & 3.7 & 2.5 & 120 & $57 \%$ \\
\hline 4 & 4.0 & 2.5 & 120 & $43 \%$ \\
\hline
\end{tabular}




\begin{tabular}{lllll}
5 & 3.5 & 3.0 & 120 & $38 \%$ \\
6 & 3.5 & 3.5 & 120 & $35 \%$ \\
7 & 3.5 & 4.0 & 120 & $25 \%$ \\
8 & 3.5 & 2.5 & 100 & $46 \%$ \\
9 & 3.5 & 3.0 & 100 & $30 \%$ \\
\hline
\end{tabular}

${ }^{a}$ Reaction conditions (unless otherwise specified): $1 \mathbf{a}\left(0.2 \mathrm{mmol}, 1.0\right.$ equiv), $\mathbf{2 b}, t-\mathrm{BuONa} \mathrm{FeI}_{2}$ (10 mol\%), 3,4,7,8-tetra-MePhen (10 mol\%), THF $(1.5 \mathrm{~mL}), 12 \mathrm{~h}$, under an argon atmosphere. ${ }^{b}$ Determined by ${ }^{1} \mathrm{H}$ NMR using mesitylene as an internal standard.

Table S12. Control Experiments of Silylation of Aryl Chlorine 1a. ${ }^{a}$

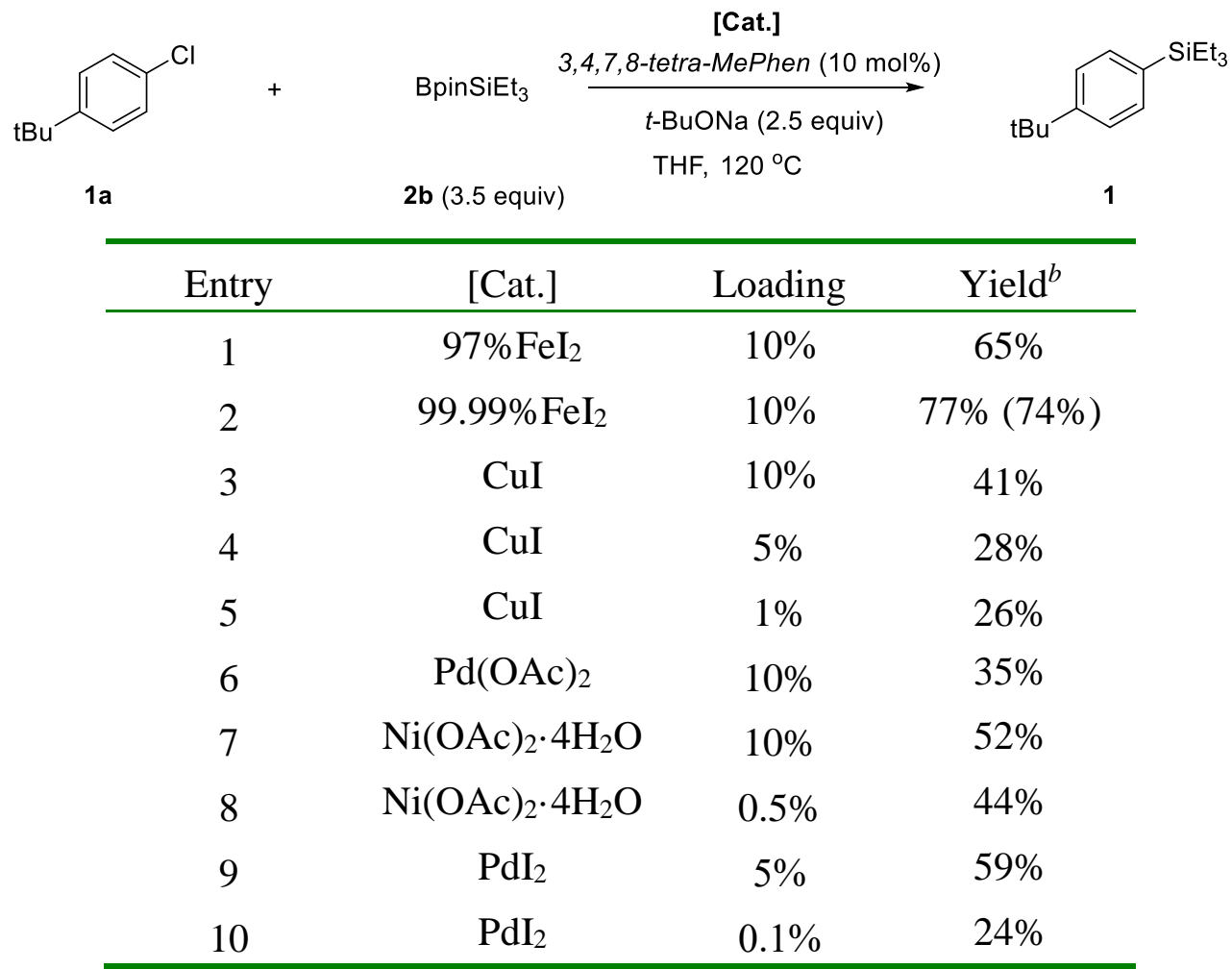

${ }^{a}$ Reaction conditions (unless otherwise specified): $1 \mathbf{1 a}(0.2 \mathrm{mmol}, 1.0$ equiv), $\mathbf{2 b}$ (3.5 equiv), $t$ BuONa (2.5 equiv), 3,4,7,8-tetra-MePhen $(10 \mathrm{~mol} \%)$, THF $(1.5 \mathrm{~mL}), 120^{\circ} \mathrm{C}, 12 \mathrm{~h}$, under an argon atmosphere. ${ }^{b}$ Determined by ${ }^{1} \mathrm{H}$ NMR using mesitylene as an internal standard.

Table S13. Evaluation of Trace Metals Present in Commercial Iron Sources.

Trace-metal analysis: $\mathrm{FeI}_{2}(99.99 \%)$, Alfa Aesar

\begin{tabular}{cc} 
Element & Molar Concentration \\
\hline $\mathrm{Cu}$ & $<0.66 \mathrm{ppm}$ \\
$\mathrm{Pd}$ & $<0.16 \mathrm{ppm}$ \\
$\mathrm{Ni}$ & $<0.67 \mathrm{ppm}$ \\
\hline
\end{tabular}




\section{Mechanistic Studies}

\section{Radical Inhibition Experiments (Table S14).}

To gain some insights into the mechanism of this iron catalyzed silylation, radical inhibition experiments were conducted (Table S14).

Table S16. Radical Inhibition Experiments of Iron-Catalyzed Silylation of 1a. ${ }^{a}$

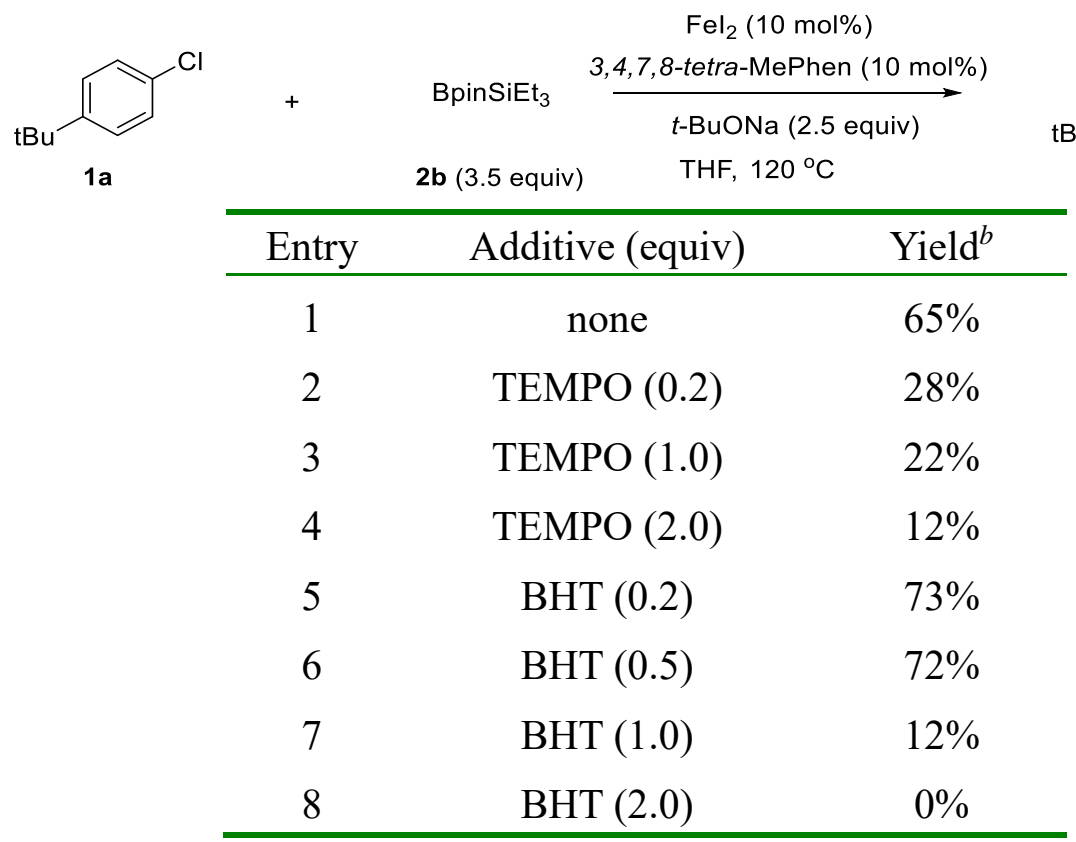

${ }^{a}$ Reaction conditions (unless otherwise specified): $1 \mathbf{1 a}(0.2 \mathrm{mmol}, 1.0$ equiv), $2 \mathbf{b}$ (3.5 equiv), $t$ BuONa (2.5 equiv), $\mathrm{FeI}_{2}$ (10 mol\%), 3,4,7,8-tetra-MePhen (10 mol\%), THF (1.5 mL), $120^{\circ} \mathrm{C}, 12$ $\mathrm{h}$, under an argon atmosphere. ${ }^{b}$ Determined by ${ }^{1} \mathrm{H}$ NMR using mesitylene as an internal standard.

Drastically diminished yields were observed when one equivalent of radical scavenger TEMPO, or radical inhibitor BHT was added under the standard silylation reaction conditions, indicating that a radical pathway might be involved.

\section{General Procedure for the Silylation of Aryl Chlorides:}

Procedure for the silylation of mono-phenyl chlorides: A $25 \mathrm{~mL}$ flame-dried Schlenk tube equipped with a magnetic stir bar was charged with $\mathrm{FeI}_{2}(6.2 \mathrm{mg}, 0.02$ mmol, 0.1 equiv), 3,4,7,8-tetra-MePhen ( $4.7 \mathrm{mg}, 0.02 \mathrm{mmol}, 0.1$ equiv), $t$-BuONa (48.1 $\mathrm{mg}, 0.5 \mathrm{mmol}, 2.5$ equiv) in glove box. Aryl chlorides $(0.2 \mathrm{mmol})$, fresh distilled silylborane $2 \mathbf{b}$ ( $186 \mu \mathrm{L}, 3.5$ equiv), fresh distilled THF ( $1.5 \mathrm{~mL})$ were then added under argon atmosphere. The reaction mixture was allowed to stir at $120{ }^{\circ} \mathrm{C}$ in oil bath for 12 h. The cooled solution was quenched with saturated $\mathrm{NH}_{4} \mathrm{Cl}$ aqueous solution, then 
diluted with ethyl acetate and washed with brine. The organic phase was dried over $\mathrm{Na}_{2} \mathrm{SO}_{4}$ and concentrated in vacuo. The residue was purified by silica gel flash chromatography to afford the corresponding compound (1-14). 


\section{Experimental Procedures and Characterization Data for Silylated Products}<smiles>CCCc1ccc(C(C)(C)C)cc1</smiles>

(4-(tert-butyl)phenyl)triethylsilane (1). This compound is known ${ }^{1}$. The product $\mathbf{1}$ (37 mg, $75 \%$ yield, $\mathrm{FeI}_{2}(99.99 \%)$ was used) as a colourless oil was purified with silica gel chromatography. ${ }^{1} \mathrm{H}$ NMR $\left(400 \mathrm{MHz}, \mathrm{CDCl}_{3}\right) \delta 7.45(\mathrm{~d}, J=8.4 \mathrm{~Hz}, 2 \mathrm{H}), 7.39(\mathrm{~d}, J=$ $8.4 \mathrm{~Hz}, 2 \mathrm{H}), 1.34(\mathrm{~s}, 9 \mathrm{H}), 0.99(\mathrm{t}, J=8.0 \mathrm{~Hz}, 9 \mathrm{H}), 0.80(\mathrm{q}, J=8.0 \mathrm{~Hz}, 6 \mathrm{H}) .{ }^{13} \mathrm{C} \mathrm{NMR}$ $\left(100 \mathrm{MHz}, \mathrm{CDCl}_{3}\right) \delta 151.6,134.2,133.9,124.7,34.7,31.4,7.6,3.6$.<smiles>CC[SiH2]c1cccc(C)c1</smiles>

Triethyl(m-tolyl)silane (2). This compound is known ${ }^{2}$. The product 2 (28 mg, 67\% yield) as a colourless oil was purified with silica gel chromatography. ${ }^{1} \mathrm{H}$ NMR (400 $\left.\mathrm{MHz}, \mathrm{CDCl}_{3}\right) \delta 7.29(\mathrm{~m}, 2 \mathrm{H}), 7.25(\mathrm{t}, J=7.6 \mathrm{~Hz}, 1 \mathrm{H}), 7.17(\mathrm{t}, J=6.4 \mathrm{~Hz}, 1 \mathrm{H}), 2.36$ $(\mathrm{s}, 3 \mathrm{H}), 0.97$ (t, $J=8.0 \mathrm{~Hz}, 9 \mathrm{H}), 0.79$ (q, $J=8.0 \mathrm{~Hz}, 6 \mathrm{H}) .{ }^{13} \mathrm{C} \mathrm{NMR}\left(100 \mathrm{MHz}, \mathrm{CDCl}_{3}\right)$ $\delta 137.4,137.0,135.0,131.4,129.7,127.7,21.8,7.6,3.5$<smiles>CCc1ccc(C)cc1</smiles>

Triethyl(p-tolyl)silane (3). This compound is known ${ }^{3}$. The product 3 (24 mg, 59\% yield) as a colourless oil was purified with silica gel chromatography. ${ }^{1} \mathrm{H}$ NMR (400 $\left.\mathrm{MHz}, \mathrm{CDCl}_{3}\right) \delta 7.39$ (d, J=7.6 Hz, $\left.2 \mathrm{H}\right), 7.18(\mathrm{~d}, J=7.6 \mathrm{~Hz}, 2 \mathrm{H}), 2.35$ (s, $\left.3 \mathrm{H}\right), 0.96$ $(\mathrm{t}, J=8.0 \mathrm{~Hz}, 9 \mathrm{H}), 0.78(\mathrm{q}, J=8.0 \mathrm{~Hz}, 6 \mathrm{H}) .{ }^{13} \mathrm{C} \mathrm{NMR}\left(100 \mathrm{MHz}, \mathrm{CDCl}_{3}\right) \delta 138.6$, $134.4,133.8,128.7,21.6,7.6,3.5$.<smiles>CC[SiH](C)c1ccc(OC)cc1</smiles>

Triethyl(4-methoxyphenyl)silane (4). This compound is known ${ }^{3}$. The product 4 (37 mg, $83 \%$ yield) as a colourless oil was purified with silica gel chromatography. ${ }^{1} \mathrm{H}$ NMR (400 MHz, $\left.\mathrm{CDCl}_{3}\right) \delta 7.43(\mathrm{~d}, J=8.4 \mathrm{~Hz}, 2 \mathrm{H}), 6.93(\mathrm{~d}, J=8.4 \mathrm{~Hz}, 2 \mathrm{H}), 3.82$ (s, 
$3 \mathrm{H}), 0.97(\mathrm{t}, J=8.0 \mathrm{~Hz}, 9 \mathrm{H}), 0.78(\mathrm{q}, J=8.0 \mathrm{~Hz}, 6 \mathrm{H}) .{ }^{13} \mathrm{C} \mathrm{NMR}\left(100 \mathrm{MHz}, \mathrm{CDCl}_{3}\right)$ $\delta 160.3,135.7,128.3,113.6,55.1,7.6,3.7$.

$\overbrace{\mathrm{BnO}}^{\mathrm{SiEt}_{3}}$

(4-(benzyloxy)phenyl)triethylsilane (5). The product 5 (34 mg, 57\% yield) as a white solid was purified with silica gel chromatography. ${ }^{1} \mathrm{H} \mathrm{NMR}\left(400 \mathrm{MHz}, \mathrm{CDCl}_{3}\right) \delta 7.47-$ 7.39 (m, $6 \mathrm{H}), 7.36$ (d, J = 6.8 Hz, $1 \mathrm{H}), 7.01$ (d, J = 8.4 Hz, 2 H), 5.09 (s, $2 \mathrm{H}), 0.98$ (t, $J=8.0 \mathrm{~Hz}, 9 \mathrm{H}), 0.79(\mathrm{q}, J=8.0 \mathrm{~Hz}, 6 \mathrm{H}) .{ }^{13} \mathrm{C} \mathrm{NMR}\left(100 \mathrm{MHz}, \mathrm{CDCl}_{3}\right) \delta 159.6,137.2$, 135.7, 128.7, 128.6, 128.1, 127.7, 114.4, 69.9, 7.6, 3.7. FTMS (EI): Calculated for $\mathrm{C}_{15} \mathrm{H}_{17} \mathrm{OSi}\left(\mathrm{M}-\mathrm{C}_{4} \mathrm{H}_{9}\right)^{+}:$241.1048; Found: 241.1043.

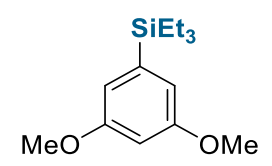

(3,5-dimethoxyphenyl)triethylsilane (6). This compound is known ${ }^{4}$. The product 6 (30 mg, 59\% yield) as a colourless oil was purified with silica gel chromatography. ${ }^{1} \mathrm{H}$ NMR (400 MHz, $\left.\mathrm{CDCl}_{3}\right) \delta 6.64(\mathrm{~s}, 1 \mathrm{H}), 6.63(\mathrm{~s}, 1 \mathrm{H}), 6.46(\mathrm{t}, J=2.0 \mathrm{~Hz}, 1 \mathrm{H}), 3.81$ $(\mathrm{s}, 6 \mathrm{H}), 0.97(\mathrm{t}, J=8.0 \mathrm{~Hz}, 9 \mathrm{H}), 0.78(\mathrm{q}, J=8.0 \mathrm{~Hz}, 6 \mathrm{H}) .{ }^{13} \mathrm{C} \mathrm{NMR}\left(100 \mathrm{MHz}, \mathrm{CDCl}_{3}\right)$ $\delta 160.4,140.1,111.9,100.6,55.3,7.6,3.5$.<smiles>CCCCC</smiles>

Benzo[d][1,3]dioxol-5-yltriethylsilane (7). This compound is known ${ }^{1}$. The product 7 (35 mg, 74\% yield) as a colourless oil was purified with silica gel chromatography. ${ }^{1} \mathrm{H}$ NMR (400 MHz, $\left.\mathrm{CDCl}_{3}\right) \delta 6.97(\mathrm{~d}, J=7.6 \mathrm{~Hz}, 1 \mathrm{H}), 6.96(\mathrm{~s}, 1 \mathrm{H}), 6.85(\mathrm{~d}, J=7.6 \mathrm{~Hz}$, $1 \mathrm{H}), 5.94(\mathrm{~s}, 2 \mathrm{H}), 0.97(\mathrm{t}, J=8.0 \mathrm{~Hz}, 9 \mathrm{H}), 0.77$ (q, $J=8.0 \mathrm{~Hz}, 6 \mathrm{H}) .{ }^{13} \mathrm{C}$ NMR $(100$ $\left.\mathrm{MHz}, \mathrm{CDCl}_{3}\right) \delta 148.3,147.4,130.4,128.2,113.6,108.7,100.5,7.5,3.7$. 
$\mathrm{SiEt}_{3}$

(4-(triethylsilyl)phenyl)methanol (8). The product $8(15 \mathrm{mg}, 34 \%$ yield) as a colourless oil was purified with silica gel chromatography. ${ }^{1} \mathrm{H} \mathrm{NMR}\left(400 \mathrm{MHz}, \mathrm{CDCl}_{3}\right)$ $\delta 7.50(\mathrm{~d}, J=8.0 \mathrm{~Hz}, 2 \mathrm{H}), 7.36(\mathrm{~d}, J=8.0 \mathrm{~Hz}, 2 \mathrm{H}), 4.69(\mathrm{~s}, 2 \mathrm{H}), 0.96(\mathrm{t}, J=8.0 \mathrm{~Hz}$, $9 \mathrm{H}), 0.79$ (q, $J=8.0 \mathrm{~Hz}, 6 \mathrm{H}) .{ }^{13} \mathrm{C} \mathrm{NMR}\left(100 \mathrm{MHz}, \mathrm{CDCl}_{3}\right) \delta 141.4,137.0,134.6$, 126.4, 65.6, 7.5, 3.5. FTMS (EI): Calculated for $\mathrm{C}_{11} \mathrm{H}_{17} \mathrm{OSi}\left(\mathrm{M}-\mathrm{C}_{2} \mathrm{H}_{5}\right)^{+}$: 193.1048; Found: 193.1042.<smiles>CCCc1ccc(N2CCOCC2)cc1</smiles>

4-(4-(triethylsilyl)phenyl)morpholine (9). The product 9 (45 $\mathrm{mg}, 81 \%$ yield) as a yelow oil was purified with silica gel chromatography. ${ }^{1} \mathrm{H}$ NMR $\left(400 \mathrm{MHz}, \mathrm{CDCl}_{3}\right) \delta$ $7.42(\mathrm{~d}, J=8.4 \mathrm{~Hz}, 2 \mathrm{H}), 6.92(\mathrm{~d}, J=8.4 \mathrm{~Hz}, 2 \mathrm{H}), 3.87(\mathrm{t}, J=4.8 \mathrm{~Hz}, 4 \mathrm{H}), 3.20$ (t, $J$ $=4.8 \mathrm{~Hz}, 4 \mathrm{H}), 0.97(\mathrm{t}, J=8.0 \mathrm{~Hz}, 9 \mathrm{H}), 0.77(\mathrm{q}, J=8.0 \mathrm{~Hz}, 6 \mathrm{H}) .{ }^{13} \mathrm{C} \mathrm{NMR}(100 \mathrm{MHz}$, $\left.\mathrm{CDCl}_{3}\right) \delta 151.5,135.4,127.0,114.8,67.1,48.8,7.6,3.6$. FTMS (ESI): Calculated for $\mathrm{C}_{16} \mathrm{H}_{28} \mathrm{NOSi}(\mathrm{M}+\mathrm{H})^{+}:$278.1934; Found: 278.1932.<smiles>CCCc1ccc(CN(C)C(=O)OC(C)(C)C)cc1</smiles>

Tert-butyl methyl(4-(triethylsilyl)benzyl)carbamate (10). The product 10 (55 mg, 82\% yield) as a yellow oil was purified with silica gel chromatography. ${ }^{1} \mathrm{H}$ NMR (400 MHz, $\left.\mathrm{CDCl}_{3}\right) \delta 7.45(\mathrm{~d}, J=7.6 \mathrm{~Hz}, 2 \mathrm{H}), 7.20(\mathrm{~d}, J=7.6 \mathrm{~Hz}, 2 \mathrm{H}), 4.41(\mathrm{~s}, 2 \mathrm{H}), 2.83(\mathrm{~s}, 3$ H), 1.48 (s, $9 \mathrm{H}), 0.96$ (t, $J=8.0 \mathrm{~Hz}, 9 \mathrm{H}), 0.79$ (q, $J=8.0 \mathrm{~Hz}, 6 \mathrm{H}) .{ }^{13} \mathrm{C}$ NMR $(100$ $\left.\mathrm{MHz}, \mathrm{CDCl}_{3}\right) \delta 156.0,138.7,136.3,134.5,126.8,79.7,52.8,34.1,28.6,7.5,3.5$. FTMS (ESI): Calculated for $\mathrm{C}_{19} \mathrm{H}_{33} \mathrm{NNaO}_{2} \mathrm{Si}(\mathrm{M}+\mathrm{Na})^{+}$: 358.2172; Found: 358.2167. 
$\mathrm{SiEt}_{\mathrm{N}}^{\mathrm{SiEt}_{3}}$

$N, N$-dimethyl-4-(triethylsilyl)aniline (11). This compound is known ${ }^{2}$. The product 11 (39 mg, 83\% yield) as a yellow oil was purified with silica gel chromatography. ${ }^{1} \mathrm{H}$ NMR (400 MHz, $\left.\mathrm{CDCl}_{3}\right) \delta 7.38(\mathrm{~d}, J=8.4 \mathrm{~Hz}, 2 \mathrm{H}), 6.75(\mathrm{~d}, J=8.4 \mathrm{~Hz}, 2 \mathrm{H}), 2.97(\mathrm{~s}$, $6 \mathrm{H}), 0.97(\mathrm{t}, J=8.0 \mathrm{~Hz}, 9 \mathrm{H}), 0.76(\mathrm{q}, J=8.0 \mathrm{~Hz}, 6 \mathrm{H}) .{ }^{13} \mathrm{C} \mathrm{NMR}\left(100 \mathrm{MHz}, \mathrm{CDCl}_{3}\right)$ $\delta 150.9,135.4,122.6,112.1,40.4,7.7,3.8$.<smiles>CC[SiH](C)c1ccc(C(F)(F)F)cc1</smiles>

Triethyl(4-(trifluoromethyl)phenyl)silane (12). This compound is known ${ }^{1}$. The product 12 (30 mg, 58\% yield) as a colourless oil was purified with silica gel chromatography. ${ }^{1} \mathrm{H}$ NMR $\left(400 \mathrm{MHz}, \mathrm{CDCl}_{3}\right) \delta$ 7.63-7.58 (m, $\left.4 \mathrm{H}\right), 0.98(\mathrm{t}, J=8.0 \mathrm{~Hz}$, $9 \mathrm{H}), 0.83(\mathrm{q}, J=8.0 \mathrm{~Hz}, 6 \mathrm{H}) .{ }^{13} \mathrm{C} \mathrm{NMR}\left(100 \mathrm{MHz}, \mathrm{CDCl}_{3}\right) \delta 142.8,134.6,130.9(\mathrm{q}$, $\left.J_{\mathrm{CF}}=32.0 \mathrm{~Hz}\right), 124.5\left(\mathrm{q}, J_{\mathrm{CF}}=270.0 \mathrm{~Hz}\right), 124.3\left(\mathrm{q}, J_{\mathrm{CF}}=4.0 \mathrm{~Hz}\right), 7.4,3.3$.<smiles>CCCc1ccc(Br)cc1</smiles>

Triethyl(4-(4,4,5,5-tetramethyl-1,3-dioxolan-2-yl)phenyl)silane

(13). This compound is known ${ }^{1}$. The product $\mathbf{1 3}$ (49 $\mathrm{mg}, \mathbf{7 7 \%}$ yield) as a white solid was purified with silica gel chromatography. ${ }^{1} \mathrm{H} \mathrm{NMR}\left(400 \mathrm{MHz}, \mathrm{CDCl}_{3}\right) \delta 7.78(\mathrm{~d}, J=7.6 \mathrm{~Hz}, 2$ H), $7.50(\mathrm{~d}, J=7.6 \mathrm{~Hz}, 2 \mathrm{H}), 1.34(\mathrm{~s}, 12 \mathrm{H}), 0.95(\mathrm{t}, J=8.0 \mathrm{~Hz}, 9 \mathrm{H}), 0.79$ (q, $J=8.0$ $\mathrm{Hz}, 6 \mathrm{H}) .{ }^{13} \mathrm{C} \mathrm{NMR}\left(100 \mathrm{MHz}, \mathrm{CDCl}_{3}\right) \delta 141.4,133.9,133.7,83.9,25.0,7.5,3.4$.<smiles>CCCc1ccc(/C=C/c2ccccc2)cc1</smiles>

(E)-triethyl(4-styrylphenyl)silane (14). This compound is known ${ }^{5}$. The product $\mathbf{1 4}$ (45 mg, 76\% yield) as a white solid was purified with silica gel chromatography. ${ }^{1} \mathrm{H}$ NMR (400 MHz, $\left.\mathrm{CDCl}_{3}\right) \delta$ 7.55-7.51 (m, $\left.6 \mathrm{H}\right), 7.38(\mathrm{t}, J=7.2 \mathrm{~Hz}, 2 \mathrm{H}), 7.29(\mathrm{~d}, J=$ 
$6.8 \mathrm{~Hz}, 1 \mathrm{H}), 7.15(\mathrm{~d}, J=4.8 \mathrm{~Hz}, 2 \mathrm{H}), 1.00(\mathrm{t}, J=8.0 \mathrm{~Hz}, 9 \mathrm{H}), 0.82(\mathrm{q}, J=8.0 \mathrm{~Hz}, 6$ H). ${ }^{13} \mathrm{C} \mathrm{NMR}\left(100 \mathrm{MHz}, \mathrm{CDCl}_{3}\right) \delta 137.7,137.5,137.2,134.7,128.94,128.90,128.8$, $127.8,126.7,125.9,7.6,3.5$.

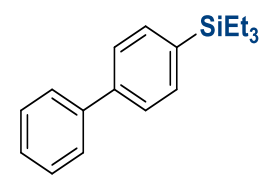

[1,1'-biphenyl]-4-yltriethylsilane (15). This compound is known ${ }^{2}$. The product 15 (48 mg, 90\% yield) as a colourless oil was purified with silica gel chromatography (Petroleum ether). ${ }^{1} \mathrm{H}$ NMR (400 MHz, $\left.\mathrm{CDCl}_{3}\right) \delta$ 7.64-7.57 (m, $\left.6 \mathrm{H}\right), 7.45(\mathrm{t}, J=8.0$ $\mathrm{Hz}, 2 \mathrm{H}), 7.36(\mathrm{t}, J=8.0 \mathrm{~Hz}, 1 \mathrm{H}), 1.02(\mathrm{t}, J=8.0 \mathrm{~Hz}, 9 \mathrm{H}), 0.84(\mathrm{q}, J=8.0 \mathrm{~Hz}, 6 \mathrm{H})$. ${ }^{13} \mathrm{C}$ NMR $\left(100 \mathrm{MHz}, \mathrm{CDCl}_{3}\right) \delta 141.6,141.3,136.4,134.8,128.9,127.4,127.3,126.6$, 7.6, 3.6.

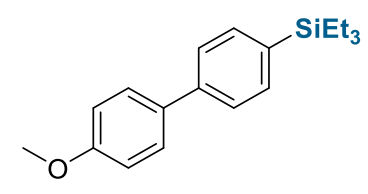

Triethyl(4'-methoxy-[1,1'-biphenyl]-4-yl)silane (16). This compound is known ${ }^{6}$. The product 16 (50 mg, 84\% yield) as a white solid was purified with silica gel chromatography. ${ }^{1} \mathrm{H}$ NMR (400 MHz, $\left.\mathrm{CDCl}_{3}\right) \delta$ 7.53-7.57 (m, $\left.6 \mathrm{H}\right), 6.99(\mathrm{~d}, J=8.8 \mathrm{~Hz}$, $2 \mathrm{H}), 3.87(\mathrm{~s}, 3 \mathrm{H}), 1.01(\mathrm{t}, J=8.0 \mathrm{~Hz}, 9 \mathrm{H}), 0.83$ (q, $J=8.0 \mathrm{~Hz}, 6 \mathrm{H}) .{ }^{13} \mathrm{C} \mathrm{NMR}(100$ $\left.\mathrm{MHz}, \mathrm{CDCl}_{3}\right) \delta 159.3,141.1,135.6,134.8,133.8,128.3,126.1,114.3,55.5,7.6,3.6$.<smiles>CC[SiH](CC)c1ccc(-c2ccc(C(C)(C)C)cc2)cc1</smiles>

(4'-(tert-butyl)-[1,1'-biphenyl]-4-yl)triethylsilane (17). This compound is known ${ }^{5}$. The product 17 (45 mg, 69\% yield) as a colourless oil was purified with silica gel chromatography. ${ }^{1} \mathrm{H}$ NMR (400 MHz, $\left.\mathrm{CDCl}_{3}\right) \delta$ 7.61-7.56 (m, $\left.6 \mathrm{H}\right), 7.49(\mathrm{~d}, J=8.4 \mathrm{~Hz}$, $2 \mathrm{H}), 1.39(\mathrm{~s}, 9 \mathrm{H}), 1.02(\mathrm{t}, J=8.0 \mathrm{~Hz}, 9 \mathrm{H}), 0.85(\mathrm{q}, J=8.0 \mathrm{~Hz}, 6 \mathrm{H}) .{ }^{13} \mathrm{C}$ NMR $(100$ 
$\left.\mathrm{MHz}, \mathrm{CDCl}_{3}\right) \delta 150.4,141.4,138.4,136.0,134.8,126.9,126.4,125.8,34.7,31.5,7.6$, 3.6.<smiles>CCCc1ccc(-c2ccc3c(c2)OCO3)cc1</smiles>

(4-(benzo[d][1,3]dioxol-5-yl)phenyl)triethylsilane (18). This compound is known ${ }^{8}$. The product 18 (42 mg, 67\% yield) as a white solid was purified with silica gel chromatography. ${ }^{1} \mathrm{H}$ NMR $\left(400 \mathrm{MHz}, \mathrm{CDCl}_{3}\right) \delta 7.53(\mathrm{q}, J=8.0 \mathrm{~Hz}, 4 \mathrm{H}), 7.10-7.08(\mathrm{~m}$, $2 \mathrm{H}), 6.89(\mathrm{~d}, J=8.0 \mathrm{~Hz}, 1 \mathrm{H}), 6.01(\mathrm{~s}, 2 \mathrm{H}), 1.00(\mathrm{t}, J=8.0 \mathrm{~Hz}, 9 \mathrm{H}), 0.83$ (q, $J=8.0$ $\mathrm{Hz}, 6 \mathrm{H}) .{ }^{13} \mathrm{C} \mathrm{NMR}\left(100 \mathrm{MHz}, \mathrm{CDCl}_{3}\right) \delta 148.2,147.2,141.2,136.0,135.7,134.8,126.2$, $120.8,108.7,107.7,101.3,7.6,3.5$.<smiles>CC[SiH](CC)c1ccc(-c2ccc(Oc3ccccc3)cc2)cc1</smiles>

Triethyl(4'-phenoxy-[1,1'-biphenyl]-4-yl)silane (19). The product 19 (58 mg, 80\% yield) as a colourless oil was purified with silica gel chromatography. ${ }^{1} \mathrm{H}$ NMR (400 $\left.\mathrm{MHz}, \mathrm{CDCl}_{3}\right) \delta 7.60-7.58(\mathrm{~m}, 6 \mathrm{H}), 7.38(\mathrm{t}, J=8.4 \mathrm{~Hz}, 2 \mathrm{H}), 7.14(\mathrm{t}, J=7.6 \mathrm{~Hz}, 1 \mathrm{H})$, 7.11-7.08 (m, $4 \mathrm{H}), 1.02(\mathrm{t}, J=8.0 \mathrm{~Hz}, 9 \mathrm{H}), 0.85(\mathrm{q}, J=8.0 \mathrm{~Hz}, 6 \mathrm{H}) .{ }^{13} \mathrm{C}$ NMR $(100$ $\left.\mathrm{MHz}, \mathrm{CDCl}_{3}\right) \delta 157.2,157.0,140.8,136.3,136.1,134.9,129.9,128.5,126.3,123.5$, 119.2, 119.1, 7.6, 3.5. FTMS (EI): Calculated for $\mathrm{C}_{22} \mathrm{H}_{23} \mathrm{OSi}\left(\mathrm{M}-\mathrm{C}_{2} \mathrm{H}_{5}\right)^{+}$: 331.1518; Found: 331.1510.<smiles>CC[SiH](CC)c1ccc(-c2ccc3ccccc3c2)cc1</smiles>

Triethyl(4-(naphthalen-2-yl)phenyl)silane (20). This compound is known ${ }^{7}$. The product 20 (36 mg, 57\% yield) as a white solid was purified with silica gel chromatography. ${ }^{1} \mathrm{H}$ NMR (400 MHz, $\left.\mathrm{CDCl}_{3}\right) \delta 8.07(\mathrm{~s}, 1 \mathrm{H}), 7.92(\mathrm{t}, J=8.0 \mathrm{~Hz}, 2 \mathrm{H})$, 
$7.77(\mathrm{~d}, J=8.8 \mathrm{~Hz}, 1 \mathrm{H}), 7.79-7.76(\mathrm{dd}, J=8.4 \mathrm{~Hz}, J=1.6 \mathrm{~Hz}, 1 \mathrm{H}), 7.73(\mathrm{~d}, J=8.0$ $\mathrm{Hz}, 2 \mathrm{H}), 7.63(\mathrm{~d}, J=8.0 \mathrm{~Hz}, 2 \mathrm{H}), 7.53-7.47$ (m, $2 \mathrm{H}), 1.03(\mathrm{t}, J=8.0 \mathrm{~Hz}, 9 \mathrm{H}), 0.86$ $(\mathrm{q}, J=8.0 \mathrm{~Hz}, 6 \mathrm{H}) .{ }^{13} \mathrm{C} \mathrm{NMR}\left(100 \mathrm{MHz}, \mathrm{CDCl}_{3}\right) \delta 141.4,138.7,136.5,134.9,133.8$, $132.8,128.5,128.4,127.8,126.8,126.4,126.1,125.9,125.7,7.6,3.6$.

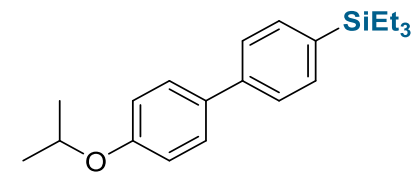

Triethyl(4'-isopropoxy-[1,1'-biphenyl]-4-yl)silane (21). The product 10 (55 mg, 85\% yield) as a white solid was purified with silica gel chromatography. ${ }^{1} \mathrm{H}$ NMR (400 MHz, $\left.\mathrm{CDCl}_{3}\right) \delta 7.54(\mathrm{~d}, J=10.0 \mathrm{~Hz}, 6 \mathrm{H}), 6.96(\mathrm{~d}, J=8.0 \mathrm{~Hz}, 2 \mathrm{H}), 4.64-4.55(\mathrm{~m}, 1 \mathrm{H}), 1.38$ $(\mathrm{d}, J=6.0 \mathrm{~Hz}, 6 \mathrm{H}), 1.00(\mathrm{t}, J=8.0 \mathrm{~Hz}, 9 \mathrm{H}), 0.83(\mathrm{q}, J=8.0 \mathrm{~Hz}, 6 \mathrm{H}) .{ }^{13} \mathrm{C}$ NMR $(100$ $\left.\mathrm{MHz}, \mathrm{CDCl}_{3}\right) \delta 157.6,141.2,135.5,134.8,133.6,128.3,126.1,116.2,70.1,22.3,7.6$, 3.6. FTMS (EI): Calculated for $\mathrm{C}_{19} \mathrm{H}_{25} \mathrm{OSi}\left(\mathrm{M}-\mathrm{C}_{2} \mathrm{H}_{5}\right)^{+}$: 297.1674; Found: 297.1667.<smiles>CCc1ccc(-c2ccc(C(F)(F)F)cc2)cc1</smiles>

Triethyl(4'-(trifluoromethyl)-[1,1'-biphenyl]-4-yl)silane (22). The product 22 (29 mg, $43 \%$ yield) as a colourless oil was purified with silica gel chromatography. ${ }^{1} \mathrm{H}$ NMR (400 MHz, $\left.\mathrm{CDCl}_{3}\right) \delta$ 7.73-7.68 (m, 4 H), 7.62-7.57 (m, $\left.4 \mathrm{H}\right), 1.00(\mathrm{t}, J=8.0 \mathrm{~Hz}$, $9 \mathrm{H}), 0.83(\mathrm{q}, J=8.0 \mathrm{~Hz}, 6 \mathrm{H}) .{ }^{13} \mathrm{C} \mathrm{NMR}\left(100 \mathrm{MHz}, \mathrm{CDCl}_{3}\right) \delta 144.9,138.5\left(\mathrm{q}, J_{\mathrm{CF}}=\right.$ $228.0 \mathrm{~Hz}), 135.0,129.5\left(\mathrm{q}, J_{\mathrm{CF}}=32.0 \mathrm{~Hz}\right), 127.5,126.6,127.5,126.6,125.84\left(\mathrm{q}, J_{\mathrm{CF}}=\right.$ 4.0 Hz), 123.1, 7.6, 3.5. FTMS (EI): Calculated for $\mathrm{C}_{17} \mathrm{H}_{18} \mathrm{~F}_{3} \mathrm{Si}\left(\mathrm{M}-\mathrm{C}_{2} \mathrm{H}_{5}\right)^{+}$: 307.1129; Found: 307.1122.

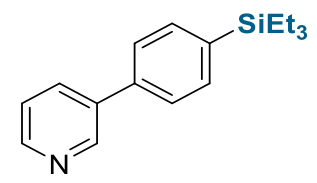


3-(4-(triethylsilyl)phenyl)pyridine (23). The product $23(28 \mathrm{mg}, 51 \%$ yield) as a yellow oil was purified with silica gel chromatography. ${ }^{1} \mathrm{H} \mathrm{NMR}\left(400 \mathrm{MHz}, \mathrm{CDCl}_{3}\right) \delta$ $8.87(\mathrm{~s}, 1 \mathrm{H}), 8.59$ (d, $J=4.8 \mathrm{~Hz}, 1 \mathrm{H}), 7.89$ (d, $J=8.0 \mathrm{~Hz}, 1 \mathrm{H}), 7.59$ (q, $J=8.0 \mathrm{~Hz}, 4$ $\mathrm{H}), 7.38-7.35(\mathrm{~m}, 1 \mathrm{H}), 1.00(\mathrm{t}, J=8.0 \mathrm{~Hz}, 9 \mathrm{H}), 0.83(\mathrm{q}, J=8.0 \mathrm{~Hz}, 6 \mathrm{H}) .{ }^{13} \mathrm{C} \mathrm{NMR}$ $\left(100 \mathrm{MHz}, \mathrm{CDCl}_{3}\right) \delta 148.6,148.5,138.1,137.6,136.8,135.1,134.5,126.5,123.7,7.6$, 3.5. FTMS (ESI): Calculated for $\mathrm{C}_{17} \mathrm{H}_{24} \mathrm{NSi}(\mathrm{M}+\mathrm{H})^{+}$: 270.1672; Found: 270.1671 .<smiles>CCCc1ccccc1-c1ccccc1</smiles>

[1,1'-biphenyl]-2-yltriethylsilane (24). This compound is known ${ }^{2}$. The product 24 (58 $\mathrm{mg}, 70 \%$ yield) as a colourless oil was purified with silica gel chromatography. ${ }^{1} \mathrm{H}$ NMR (400 MHz, $\left.\mathrm{CDCl}_{3}\right) \delta 7.77(\mathrm{~d}, J=6.8 \mathrm{~Hz}, 1 \mathrm{H}), 7.40-7.36(\mathrm{~m}, 5 \mathrm{H}), 7.30-7.28(\mathrm{~m}$, $2 \mathrm{H}), 7.23(\mathrm{~d}, J=7.2 \mathrm{~Hz}, 1 \mathrm{H}), 0.82(\mathrm{t}, J=8.0 \mathrm{~Hz}, 9 \mathrm{H}), 0.48(\mathrm{q}, J=8.0 \mathrm{~Hz}, 6 \mathrm{H}) .{ }^{13} \mathrm{C}$ NMR $\left(100 \mathrm{MHz} \mathrm{CDCl}_{3}\right) \delta 149.8,144.7,135.9,135.3,129.8,129.3,128.4,127.7,127.2$, $126.2,7.7,4.4$.<smiles>CC[SiH2]c1ccc(OC)nc1</smiles>

2-methoxy-5-(triethylsilyl)pyridine (25). The product 25 (33 $\mathrm{mg}, 74 \%$ yield) as a yellow oil was purified with silica gel chromatography. ${ }^{1} \mathrm{H} \mathrm{NMR}\left(400 \mathrm{MHz}, \mathrm{CDCl}_{3}\right) \delta$ $8.22(\mathrm{~s}, 1 \mathrm{H}), 7.63(\mathrm{dd}, J=8.0 \mathrm{~Hz}, J=2.0 \mathrm{~Hz}, 1 \mathrm{H}), 6.74(\mathrm{~d}, J=8.0 \mathrm{~Hz}, 1 \mathrm{H}), 3.94(\mathrm{~s}$, $3 \mathrm{H}), 0.96(\mathrm{t}, J=8.0 \mathrm{~Hz}, 9 \mathrm{H}), 0.77(\mathrm{q}, J=8.0 \mathrm{~Hz}, 6 \mathrm{H}) .{ }^{13} \mathrm{C} \mathrm{NMR}\left(100 \mathrm{MHz}, \mathrm{CDCl}_{3}\right)$ $\delta 164.8,152.4,144.3,123.4,110.8,53.3,7.4,3.5$. FTMS (ESI): Calculated for $\mathrm{C}_{12} \mathrm{H}_{22} \mathrm{NOSi}(\mathrm{M}+\mathrm{H})^{+}:$224.1465; Found: 224.1465.<smiles>CC[SiH2]c1cccnc1</smiles>

3-(triethylsilyl)pyridine (26). This compound is known ${ }^{5}$. The product 26 (23 mg, 60\% yield) as a yellow oil was purified with silica gel chromatography. ${ }^{1} \mathrm{H}$ NMR (400 MHz, 
$\left.\mathrm{CDCl}_{3}\right) \delta 8.65(\mathrm{~s}, 1 \mathrm{H}), 8.57(\mathrm{~d}, J=3.6 \mathrm{~Hz}, 1 \mathrm{H}), 7.76(\mathrm{~d}, J=3.6 \mathrm{~Hz}, 1 \mathrm{H}), 7.25(\mathrm{t}, J=$ $7.6 \mathrm{~Hz}, 1 \mathrm{H}), 0.96(\mathrm{t}, J=8.0 \mathrm{~Hz}, 9 \mathrm{H}), 0.81$ (q, $J=8.0 \mathrm{~Hz}, 6 \mathrm{H}) .{ }^{13} \mathrm{C} \mathrm{NMR}(100 \mathrm{MHz}$, $\left.\mathrm{CDCl}_{3}\right) \delta 154.6,149.9,142.1,132.5,123.4,7.4,3.2$.<smiles>CC[SiH](CC)c1ccc2[nH]ccc2c1</smiles>

5-(triethylsilyl)-1 $\mathrm{H}$-indole (28). The product 28 (20 $\mathrm{mg}, 44 \%$ yield) as a yellow oil was purified with silica gel chromatography. ${ }^{1} \mathrm{H} \mathrm{NMR}\left(400 \mathrm{MHz}, \mathrm{CDCl}_{3}\right) \delta 8.12(\mathrm{~s}, 1$ H), $7.82(\mathrm{~s}, 1 \mathrm{H}), 7.41(\mathrm{~d}, J=8.0 \mathrm{~Hz}, 1 \mathrm{H}), 7.32(\mathrm{~d}, J=8.0 \mathrm{~Hz}, 1 \mathrm{H}), 7.19(\mathrm{~s}, 1 \mathrm{H}), 6.57$ $(\mathrm{s}, 1 \mathrm{H}), 1.00(\mathrm{t}, J=8.0 \mathrm{~Hz}, 9 \mathrm{H}), 0.85(\mathrm{q}, J=8.0 \mathrm{~Hz}, 6 \mathrm{H}) .{ }^{13} \mathrm{C} \mathrm{NMR}\left(100 \mathrm{MHz}, \mathrm{CDCl}_{3}\right)$ $\delta 136.5,127.9,127.7,127.4,127.0,124.0,110.7,102.7,7.7,3.9$. FTMS (EI): Calculated for $\mathrm{C}_{12} \mathrm{H}_{16} \mathrm{NSi}\left(\mathrm{M}-\mathrm{C}_{2} \mathrm{H}_{5}\right)^{+}$: 202.1052; Found: 202.1045 .<smiles>CC[SiH](C)c1ccc2c(ccn2C(=O)OC(C)(C)C)c1</smiles>

Tert-butyl 5-(triethylsilyl)-1H-indole-1-carboxylate (29). The product 29 (35 mg, 53\% yield) as a yellow oil was purified with silica gel chromatography. ${ }^{1} \mathrm{H}$ NMR $(400 \mathrm{MHz}$, $\left.\mathrm{CDCl}_{3}\right) \delta 8.12(\mathrm{~d}, J=8.0 \mathrm{~Hz}, 1 \mathrm{H}), 7.70(\mathrm{~s}, 1 \mathrm{H}), 7.59(\mathrm{~d}, J=4.0 \mathrm{~Hz}, 1 \mathrm{H}), 7.43(\mathrm{~d}, J=$ $8.0 \mathrm{~Hz}, 1 \mathrm{H}), 6.58(\mathrm{~d}, J=4.0 \mathrm{~Hz}, 1 \mathrm{H}), 1.67(\mathrm{~s}, 9 \mathrm{H}), 0.98$ (t, $J=8.0 \mathrm{~Hz}, 9 \mathrm{H}), 0.83$ (q, $J=8.0 \mathrm{~Hz}, 6 \mathrm{H}) .{ }^{13} \mathrm{C} \mathrm{NMR}\left(100 \mathrm{MHz}, \mathrm{CDCl}_{3}\right) \delta 150.0,135.8,130.7,130.5,130.1$, $127.3,125.8,114.7,107.5,83.8,28.4,7.6,3.8$.<smiles>CCCc1ccc(C(c2ccccc2)N2CCN(C(=O)OC(C)(C)C)CC2)cc1</smiles>

Tert-butyl 4-(phenyl(4-(triethylsilyl)phenyl)methyl)piperazine-1-carboxylate (30). The product 30 (72 $\mathrm{mg}, 77 \%$ yield) as a yellow oil was purified with silica gel chromatography. ${ }^{1} \mathrm{H}$ NMR $\left(400 \mathrm{MHz}, \mathrm{CDCl}_{3}\right) \delta 7.42(\mathrm{~d}, J=7.2 \mathrm{~Hz}, 1 \mathrm{H}), 7.38-7.35(\mathrm{~m}$, 
$4 \mathrm{H}), 7.28$ (t, $J=7.6 \mathrm{~Hz}, 2 \mathrm{H}), 7.19(\mathrm{t}, J=7.2 \mathrm{~Hz}, 1 \mathrm{H}), 4.20(\mathrm{~s}, 1 \mathrm{H}), 3.42(\mathrm{~s}, 4 \mathrm{H}), 2.33$ $(\mathrm{s}, 4 \mathrm{H}), 1.44(\mathrm{~s}, 9 \mathrm{H}), 0.94(\mathrm{t}, J=8.0 \mathrm{~Hz}, 9 \mathrm{H}), 0.75(\mathrm{q}, J=8.0 \mathrm{~Hz}, 6 \mathrm{H}) .{ }^{13} \mathrm{C} \mathrm{NMR}$ $\left(100 \mathrm{MHz}, \mathrm{CDCl}_{3}\right) \delta 155.0,142.9,142.6,136.2,134.6,128.6,128.1,127.3,127.2,79.6$, 76.3, 51.9, 44.7-43.1 (m, 1 C), 28.6, 7.6, 3.5. FTMS (ESI): Calculated for $\mathrm{C}_{28} \mathrm{H}_{43} \mathrm{~N}_{2} \mathrm{O}_{2} \mathrm{Si}$ $(\mathrm{M}+\mathrm{H})^{+}$: 467.3088; Found: 467.3080.

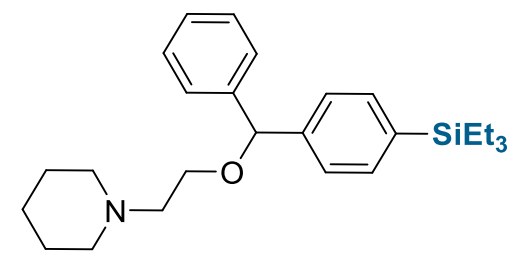

1-(2-(phenyl(4-(triethylsilyl)phenyl)methoxy)ethyl)piperidine (31). This compound is known. The product $\mathbf{3 1}$ (72 $\mathrm{mg}, 88 \%$ yield) as a yellow oil was purified with silica gel chromatography. ${ }^{1} \mathrm{H} \mathrm{NMR}\left(400 \mathrm{MHz}, \mathrm{CDCl}_{3}\right) \delta 7.43(\mathrm{~d}, J=8.0 \mathrm{~Hz}, 2 \mathrm{H}), 7.37-7.30$ $(\mathrm{m}, 6 \mathrm{H}), 7.23(\mathrm{~d}, J=8.0 \mathrm{~Hz}, 1 \mathrm{H}), 5.36(\mathrm{~s}, 1 \mathrm{H}), 3.61(\mathrm{t}, J=6.4 \mathrm{~Hz}, 2 \mathrm{H}), 2.66(\mathrm{t}, J=$ $6.4 \mathrm{~Hz}, 2 \mathrm{H}), 2.45$ (s, $4 \mathrm{H}), 1.60-1.55$ (m, $4 \mathrm{H}), 1.45-1.40$ (m, $2 \mathrm{H}), 0.95$ (t, $J=8.0 \mathrm{~Hz}$, $9 \mathrm{H}), 0.76(\mathrm{q}, J=8.0 \mathrm{~Hz}, 6 \mathrm{H}) .{ }^{13} \mathrm{C} \mathrm{NMR}\left(100 \mathrm{MHz}, \mathrm{CDCl}_{3}\right) \delta 142.8,142.4,136.5$, $134.4,128.5,127.5,127.2,126.4,84.2,67.2,58.8,55.1,26.1,24.4,7.5,3.5$. FTMS (ESI): Calculated for $\mathrm{C}_{26} \mathrm{H}_{40} \mathrm{NOSi}(\mathrm{M}+\mathrm{H})^{+}$: 410.2873; Found: 410.2871 .

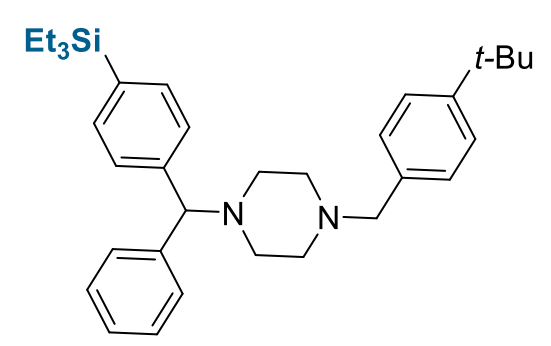

1-(4-(tert-butyl)benzyl)-4-(phenyl(4-(triethylsilyl)phenyl)methyl)piperazine (32).

The product 32 (54 mg, 53\% yield) as a white solid was purified with silica gel chromatography. ${ }^{1} \mathrm{H}$ NMR (400 MHz, $\left.\mathrm{CDCl}_{3}\right) \delta 7.44(\mathrm{~d}, J=7.6 \mathrm{~Hz}, 2 \mathrm{H}), 7.39$ (s, $\left.4 \mathrm{H}\right)$, $7.33(\mathrm{~d}, J=8.0 \mathrm{~Hz}, 2 \mathrm{H}), 7.28(\mathrm{t}, J=7.6 \mathrm{~Hz}, 2 \mathrm{H}), 7.24(\mathrm{~d}, J=8.0 \mathrm{~Hz}, 2 \mathrm{H}), 7.19(\mathrm{t}, J$ $=7.2 \mathrm{~Hz}, 1 \mathrm{H}), 4.23(\mathrm{~s}, 1 \mathrm{H}), 3.55-3.48(\mathrm{~m}, 2 \mathrm{H}), 2.50-2.45(\mathrm{~m}, 8 \mathrm{H}), 1.32(\mathrm{~s}, 9 \mathrm{H}), 0.96$ $(\mathrm{t}, J=8.0 \mathrm{~Hz}, 9 \mathrm{H}), 0.77(\mathrm{q}, J=8.0 \mathrm{~Hz}, 6 \mathrm{H}) .{ }^{13} \mathrm{C} \mathrm{NMR}\left(100 \mathrm{MHz}, \mathrm{CDCl}_{3}\right) \delta 150.0$, $143.2,142.9,135.8,135.0,134.4,129.2,128.5,128.2,127.4,127.0,125.2,76.5,62.9$, 
53.5, 52.1, 34.6, 31.5, 7.6, 3.5. FTMS (ESI): Calculated for $\mathrm{C}_{34} \mathrm{H}_{49} \mathrm{~N}_{2} \mathrm{Si}(\mathrm{M}+\mathrm{H})^{+}$: 513.3659; Found: 513.36493.

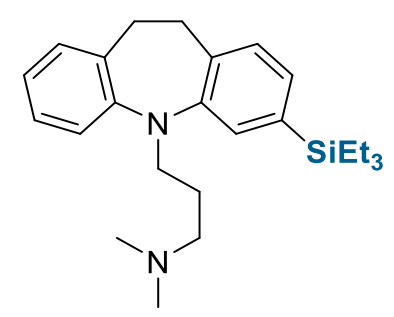

\section{$N, N$-dimethyl-3-(3-(triethylsilyl)-10,11-dihydro-5H-dibenzo[b,f]azepin-5-}

yl)propan-1-amine(33). The product $33(42.3 \mathrm{mg}, 54 \%$ yield) as a yellow oil was purified with silica gel chromatography. ${ }^{1} \mathrm{H}$ NMR $\left(400 \mathrm{MHz}, \mathrm{CDCl}_{3}\right) \delta 7.17(\mathrm{~s}, 1 \mathrm{H})$, 7.13-7.07 (m, $4 \mathrm{H}), 7.03$ (d, $J=7.2 \mathrm{~Hz}, 1 \mathrm{H}), 6.91(\mathrm{t}, J=7.2 \mathrm{~Hz}, 1 \mathrm{H}), 3.80(\mathrm{t}, J=8.0$ Hz, 2 H), 3.15 (s, 4 H), 2.36 (t, J = 8.0 Hz, 2 H), 2.17 (s, $6 \mathrm{H}), 1.80-1.72$ (m, 2 H), 0.97 $(\mathrm{t}, J=8.0 \mathrm{~Hz}, 9 \mathrm{H}), 0.77(\mathrm{q}, J=8.0 \mathrm{~Hz}, 6 \mathrm{H}) .{ }^{13} \mathrm{C} \mathrm{NMR}\left(100 \mathrm{MHz}, \mathrm{CDCl}_{3}\right) \delta 148.6$, 147.6, 135.2, 135.0, 134.4, 129.9, 129.4, 128.4, 126.5, 125.6, 122.5, 120.2, 57.8, 49.0, 45.5, 32.4, 26.0, 25.0, 7.6, 3.6. FTMS (ESI): Calculated for $\mathrm{C}_{25} \mathrm{H}_{39} \mathrm{~N}_{2} \mathrm{Si}(\mathrm{M}+\mathrm{H})^{+}$: 395.2877; Found: 395.2858.

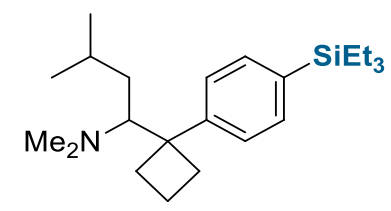

N,N,3-trimethyl-1-(1-(4-(triethylsilyl)phenyl)cyclobutyl)butan-1-amine (34). The product 34 (43.1 mg, 60\% yield) as a yellow oil was purified with silica gel chromatography. ${ }^{1} \mathrm{H}$ NMR $\left(400 \mathrm{MHz}, \mathrm{CDCl}_{3}\right) \delta 7.36(\mathrm{~d}, J=8.0 \mathrm{~Hz}, 2 \mathrm{H}), 7.19(\mathrm{~d}, J=$ $8.0 \mathrm{~Hz}, 2 \mathrm{H}), 2.90$ (dd, $J=10.4 \mathrm{~Hz}, J=2.8 \mathrm{~Hz}, 2 \mathrm{H}), 2.45-2.34(\mathrm{~m}, 2 \mathrm{H}), 2.27-2.20$ (m, $1 \mathrm{H}), 2.13$ (s, $6 \mathrm{H}), 1.96-1.89$ (m, $1 \mathrm{H}), 1.81-1.72(\mathrm{~m}, 1 \mathrm{H}), 1.62-1.48$ (m, $1 \mathrm{H}), 1.30-$ $1.21(\mathrm{~m}, 1 \mathrm{H}), 1.13-1.06(\mathrm{~m}, 1 \mathrm{H}), 0.96(\mathrm{t}, J=8.0 \mathrm{~Hz}, 11 \mathrm{H}), 0.88(\mathrm{~d}, J=6.8 \mathrm{~Hz}, 3 \mathrm{H})$, $0.77(\mathrm{q}, J=8.0 \mathrm{~Hz}, 6 \mathrm{H}) .{ }^{13} \mathrm{C} \mathrm{NMR}\left(100 \mathrm{MHz}, \mathrm{CDCl}_{3}\right) \delta 148.3,133.5,133.1,127.3$, $67.9,52.0,44.0,36.1,33.4,33.0,26.3,24.3,21.6,15.9,7.6,3.5$. 


\section{Reference}

1. Zarate, C.; Martin R. J. Am. Chem. Soc. 2014, 136: 2236-2239.

2. Zarate, C.; Nakajima, M.; Martin, R. J. Am. Chem. Soc. 2017, 139, 1191-1197.

3. Wang, X.; Wang, Z.; Nishihara, Y. Chem. Commun. 2019, 55, 10507-10510.

4. Murai. M.; Takami. K.; Takai, K. Chem. Eur. J. 2015, 21, 4566-4570.

5. Guo, L.; Chatupheeraphat, A.; Rueping, M. Angew. Chem. Int. Ed. 2016, 55, 1181011813.

6. Liu, X.; Zarate, C.; Martin, R. Angew. Chem. Int. Ed. 2019, 58, 2064-2068.

7. Pu, X.; Hu, J.; Zhao, Y.; Shi, Z. ACS Catal. 2016, 6, 6692-6698. 
(4-(tert-butyl)phenyl)triethylsilane (1).
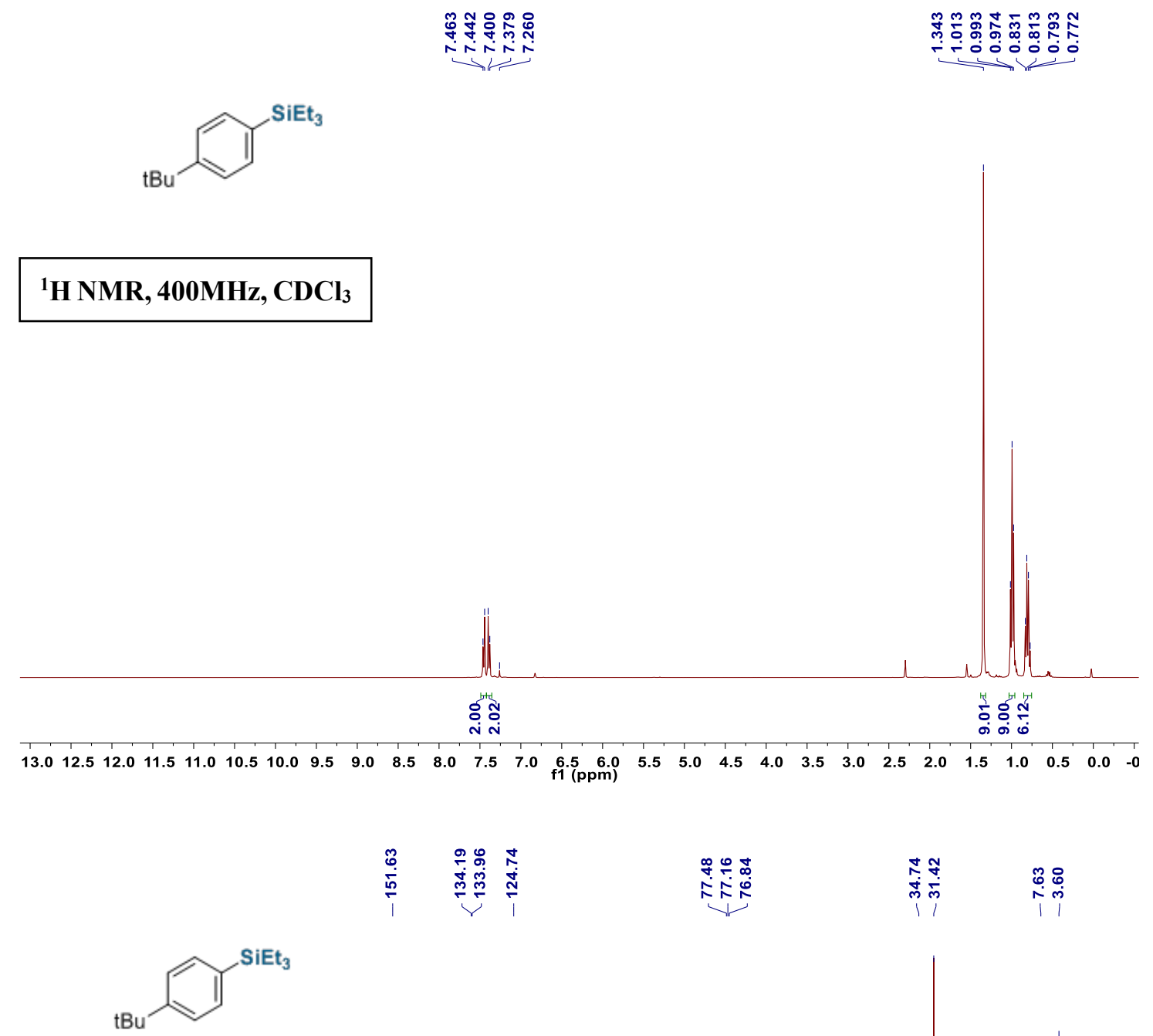

${ }^{13} \mathrm{C}$ NMR, 100MHz, $\mathrm{CDCl}_{3}$

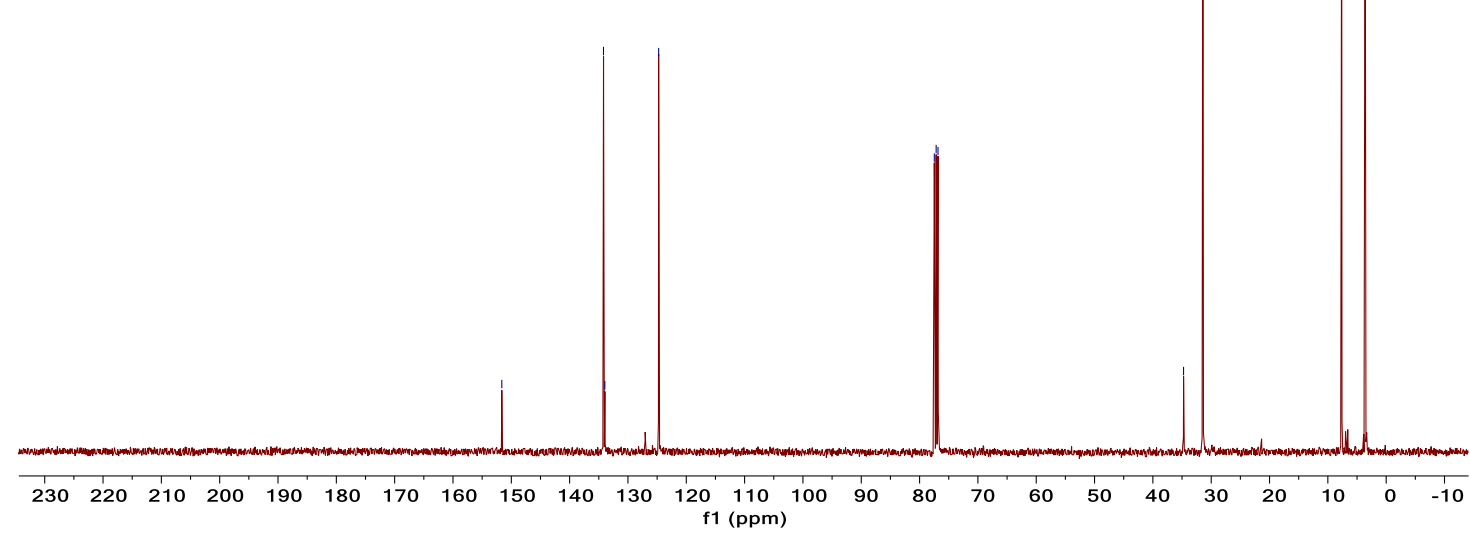


Triethyl(m-tolyl)silane (2).
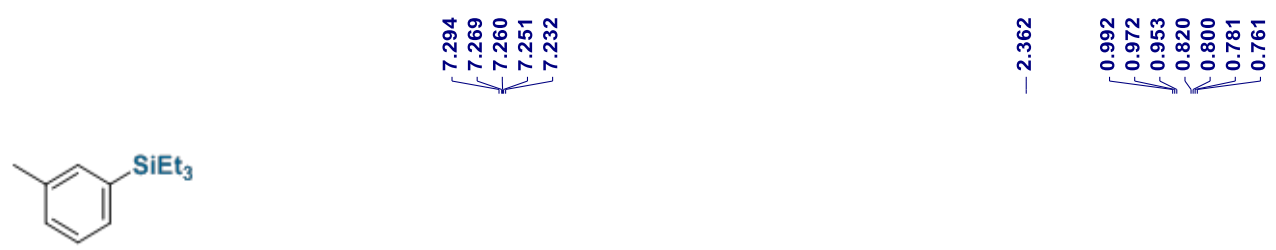

${ }^{1} \mathrm{H}$ NMR, 400MHz, $\mathrm{CDCl}_{3}$

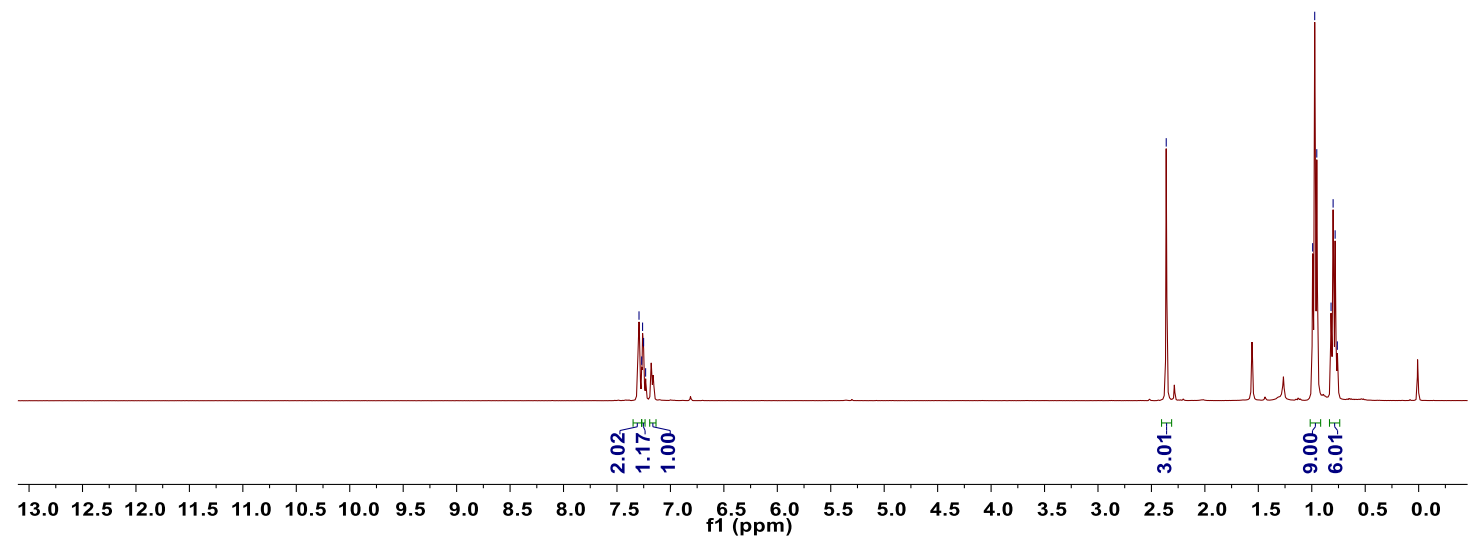

\begin{tabular}{|c|c|c|}
\hline 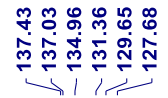 & 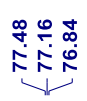 & 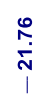 \\
\hline
\end{tabular}

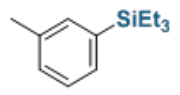

${ }^{13} \mathrm{C}$ NMR, 100MHz, $\mathrm{CDCl}_{3}$

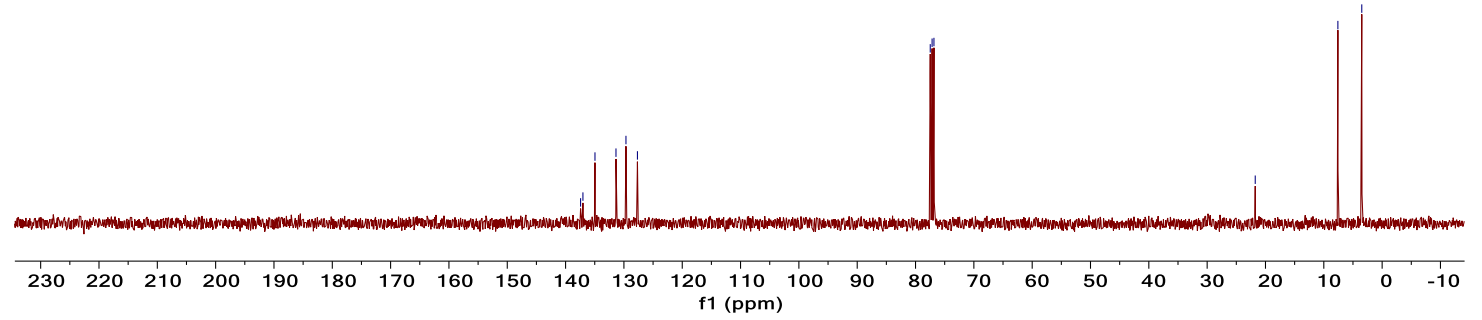


Triethyl( $p$-tolyl)silane (3).<smiles>CC[SiH2]c1ccc(C)cc1</smiles>

${ }^{1} \mathrm{H}$ NMR, $400 \mathrm{MHz}, \mathrm{CDCl}_{3}$

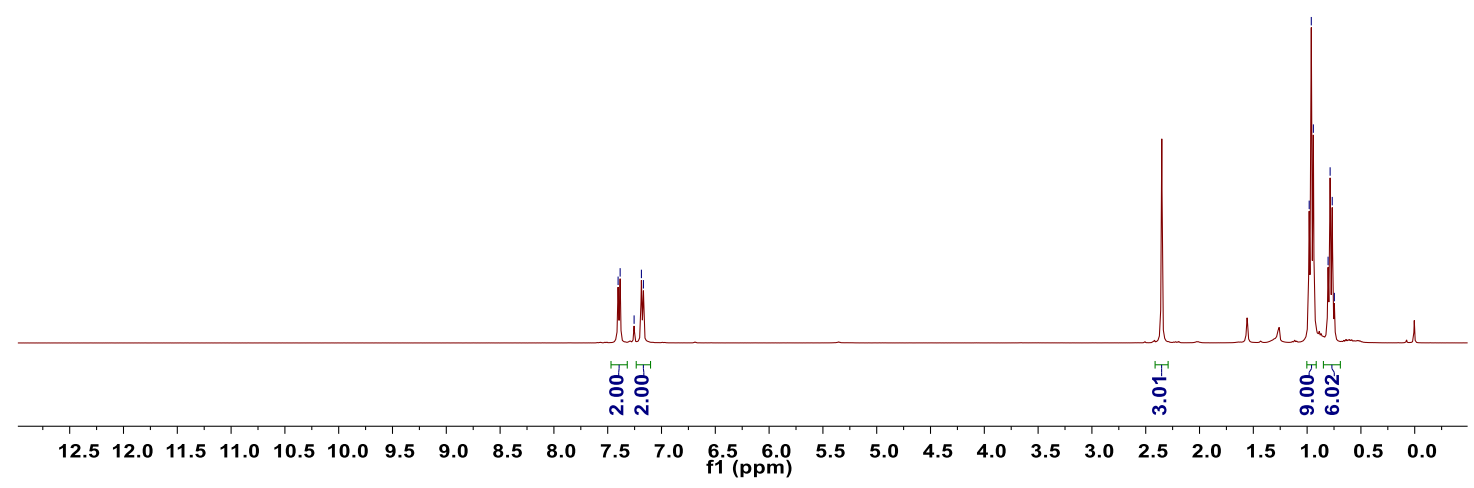

\begin{tabular}{|c|c|c|}
\hline 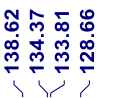 & 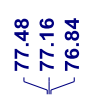 & 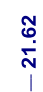 \\
\hline
\end{tabular}

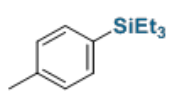

${ }^{13} \mathrm{C}$ NMR, $100 \mathrm{MHz}, \mathrm{CDCl}_{3}$

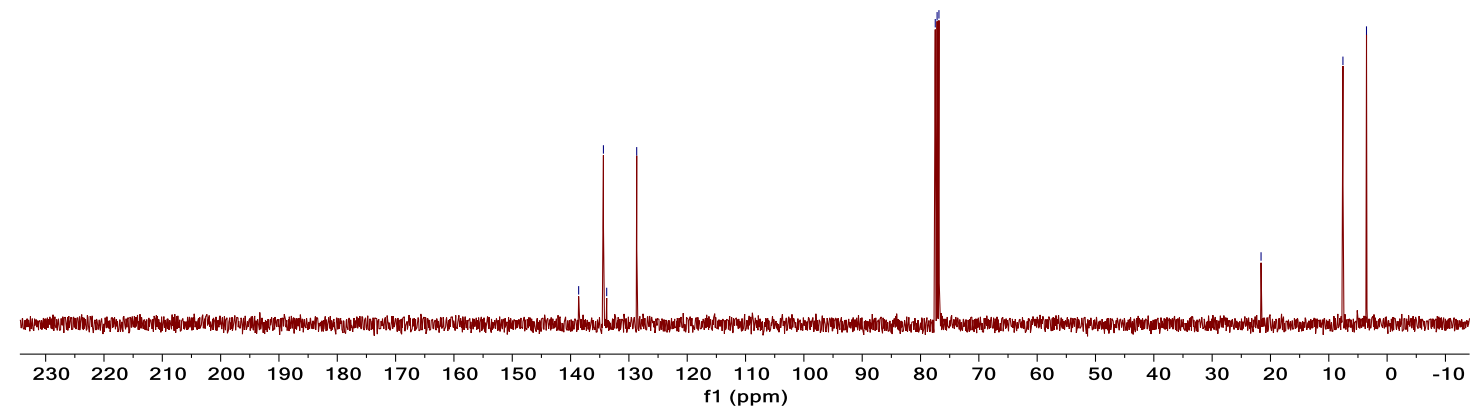


Triethyl(4-methoxyphenyl)silane (4).
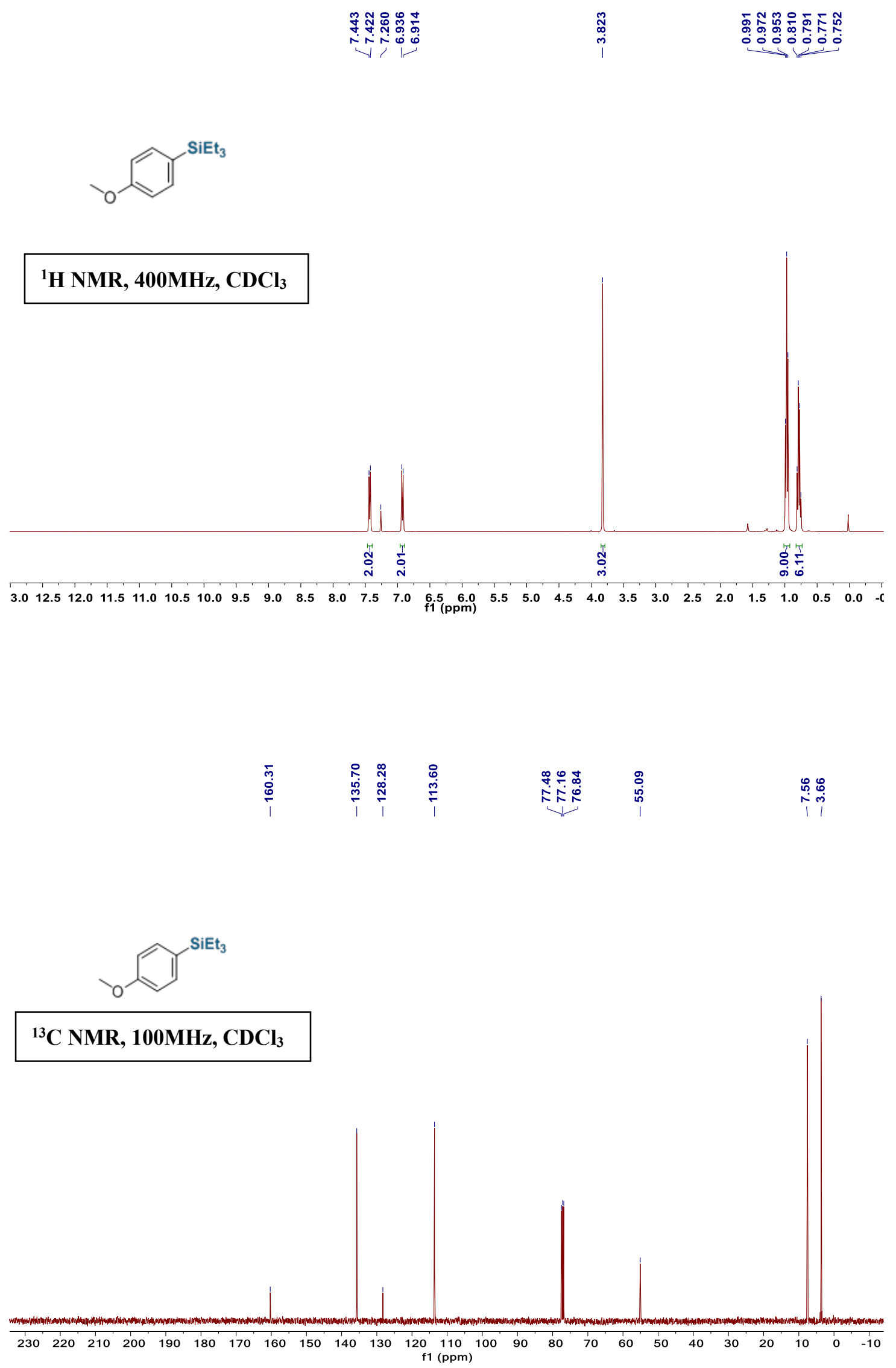
(4-(benzyloxy)phenyl)triethylsilane (5).

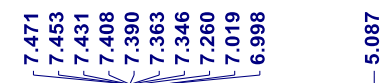

勿

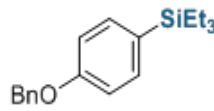

${ }^{1} \mathrm{H}$ NMR, 400MHz, $\mathrm{CDCl}_{3}$
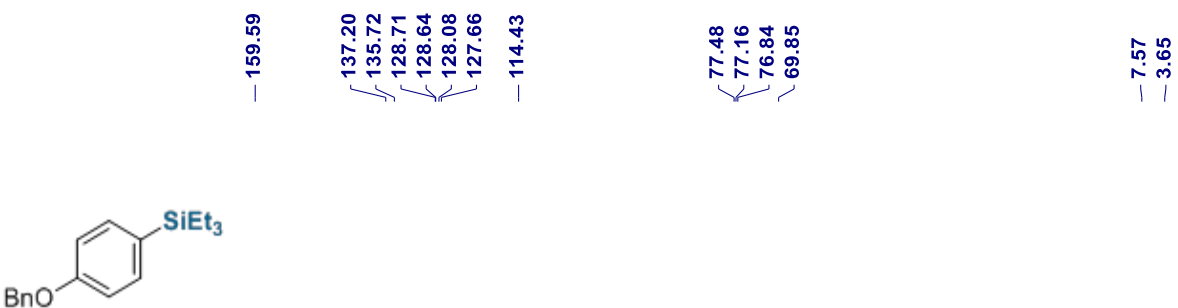

${ }^{13} \mathrm{C}$ NMR, $100 \mathrm{MHz}, \mathrm{CDCl}_{3}$

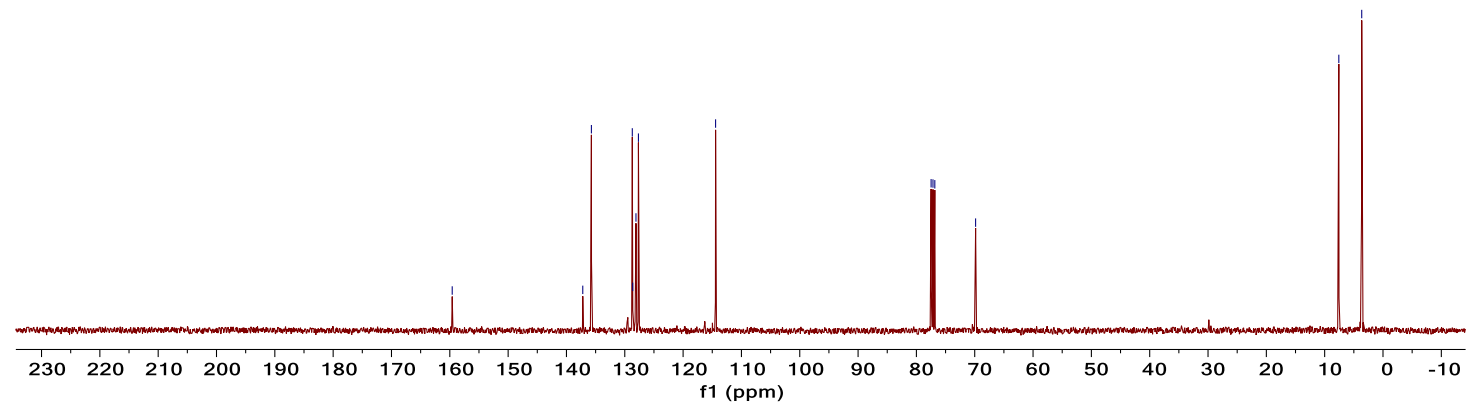


(3,5-dimethoxyphenyl)triethylsilane (6).

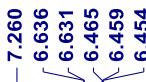

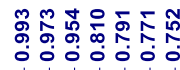<smiles>CCCCCCCCCCCCC</smiles>

${ }^{1} \mathrm{H}$ NMR, 400MHz, $\mathrm{CDCl}_{3}$

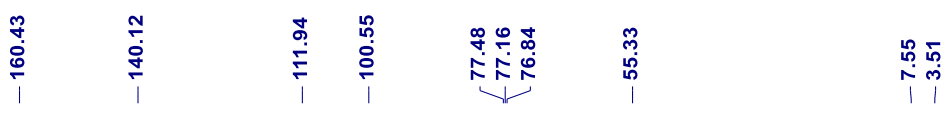

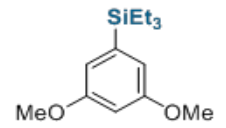

${ }^{13} \mathrm{C}$ NMR, 100MHz, $\mathrm{CDCl}_{3}$

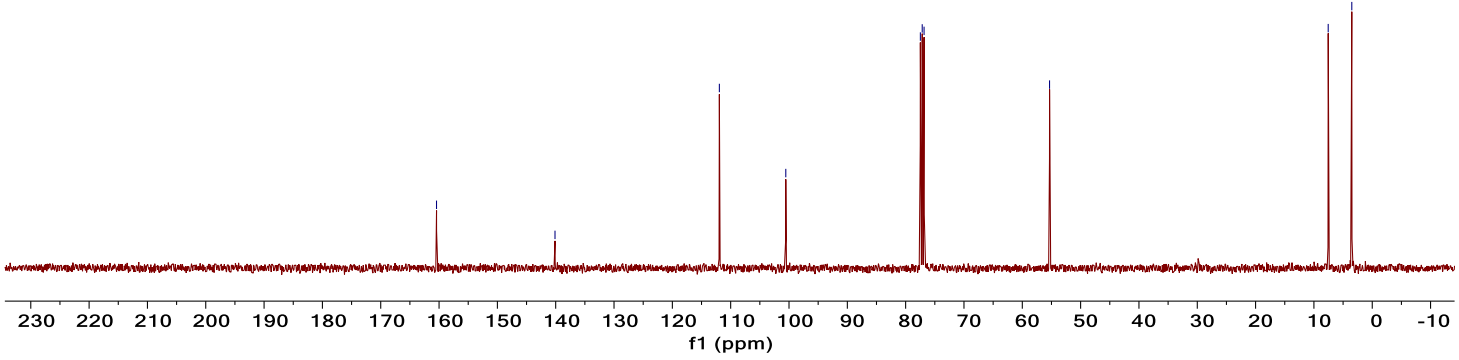


Benzo[d][1,3]dioxol-5-yltriethylsilane (7).

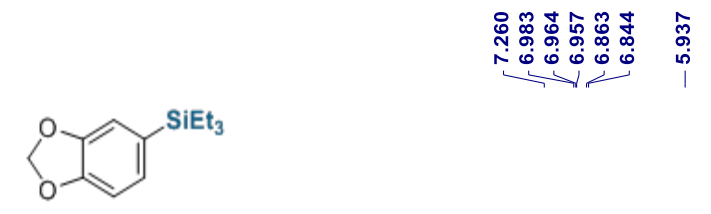

${ }^{1} \mathrm{H}$ NMR, 400MHz, $\mathrm{CDCl}_{3}$

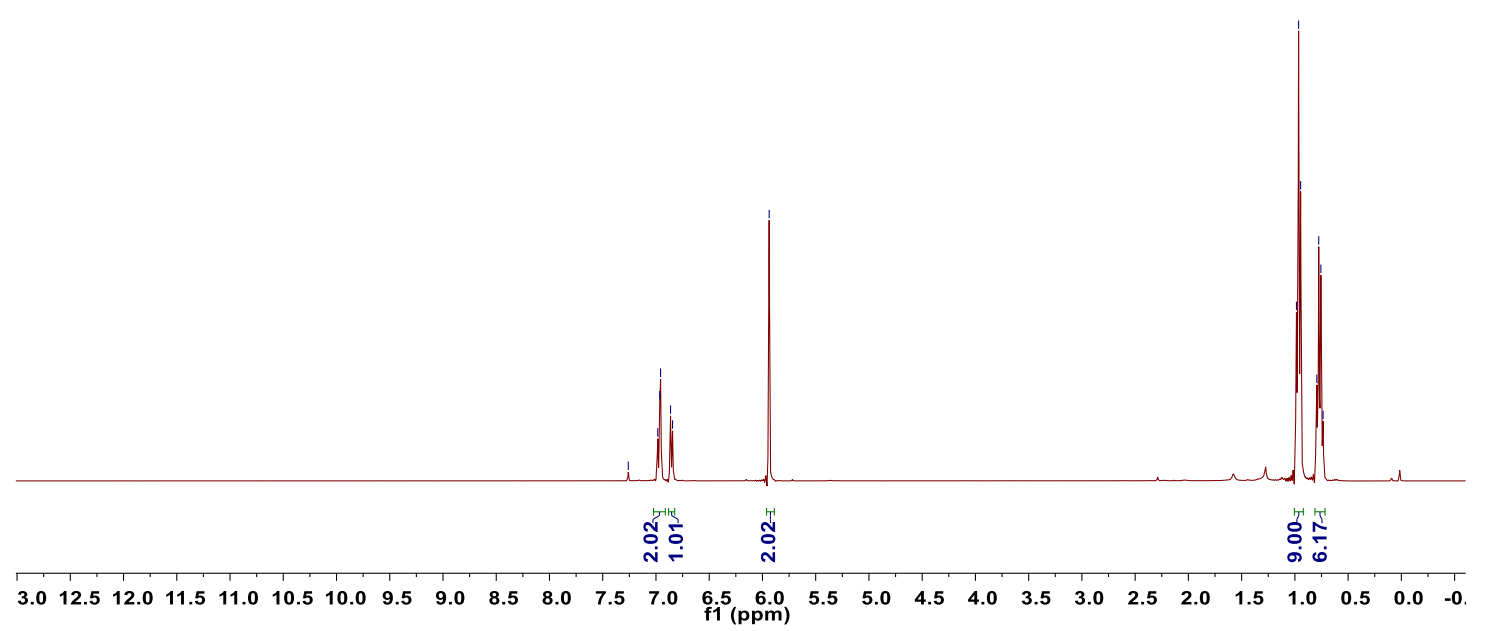

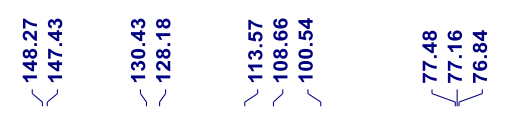

inco

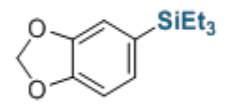

${ }^{13} \mathrm{C}$ NMR, $100 \mathrm{MHz}, \mathrm{CDCl}_{3}$

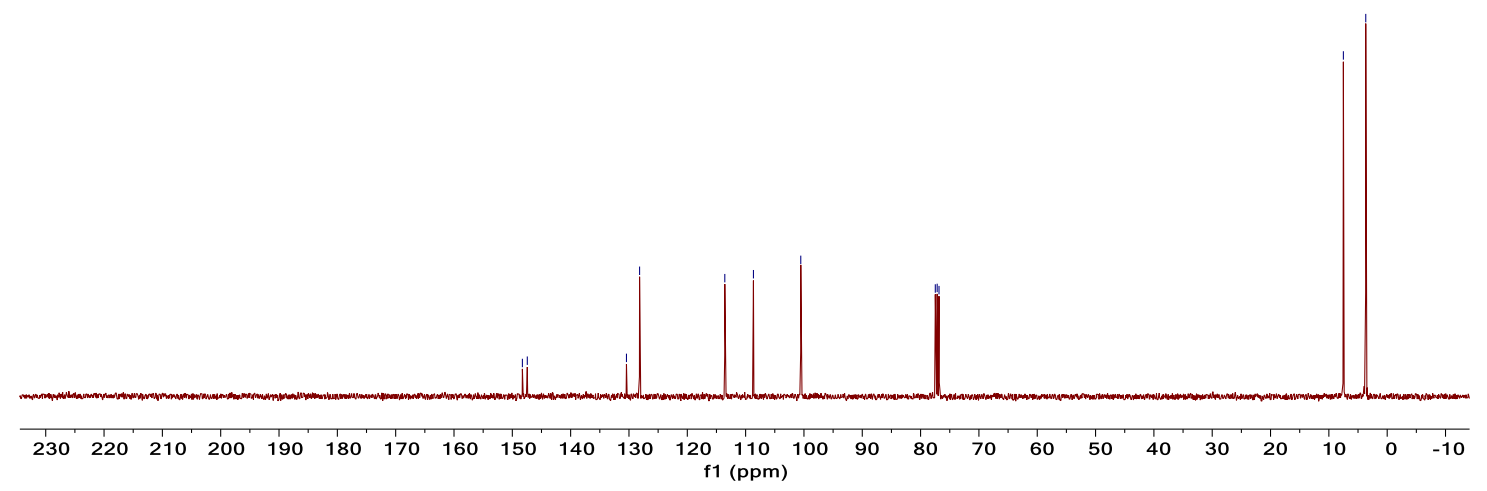


(4-(triethylsilyl)phenyl)methanol (8).

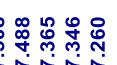

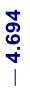

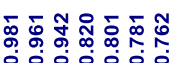

令然

०0ं0 000

SiEt

${ }^{1} \mathrm{H}$ NMR, 400MHz, $\mathrm{CDCl}_{3}$

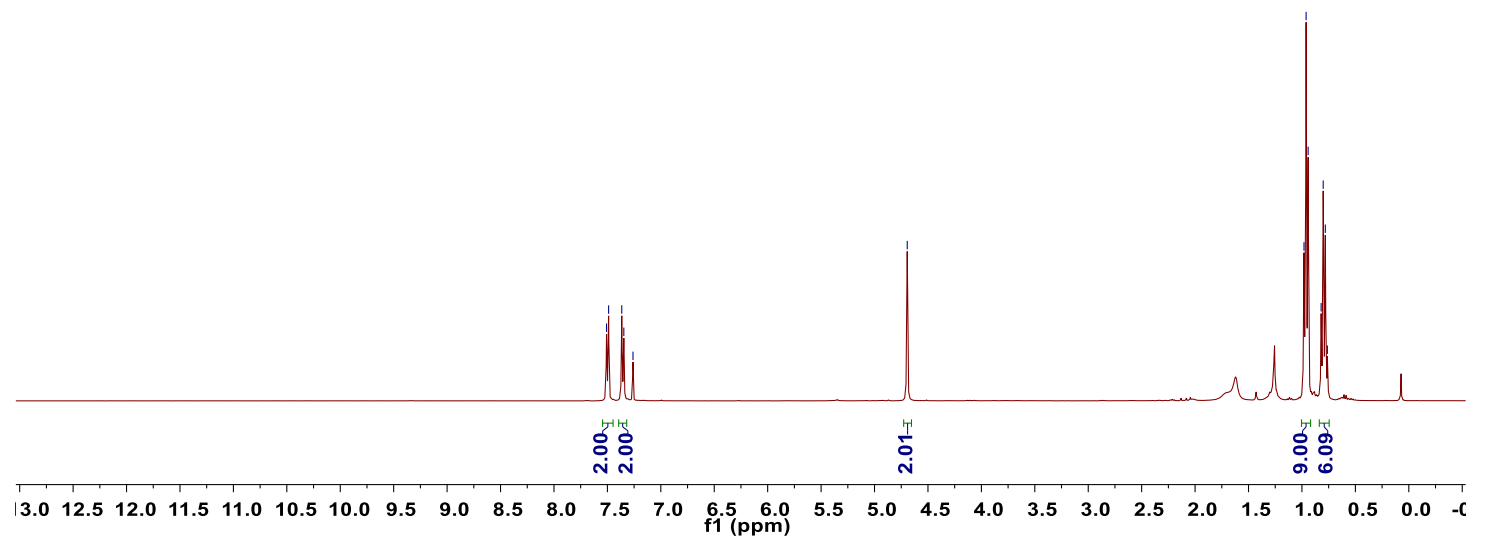

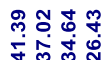

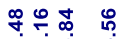

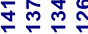

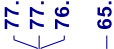

กึ?

$\mathrm{SiEt}_{3}$

${ }^{13} \mathrm{C}$ NMR, $100 \mathrm{MHz}, \mathrm{CDCl}_{3}$

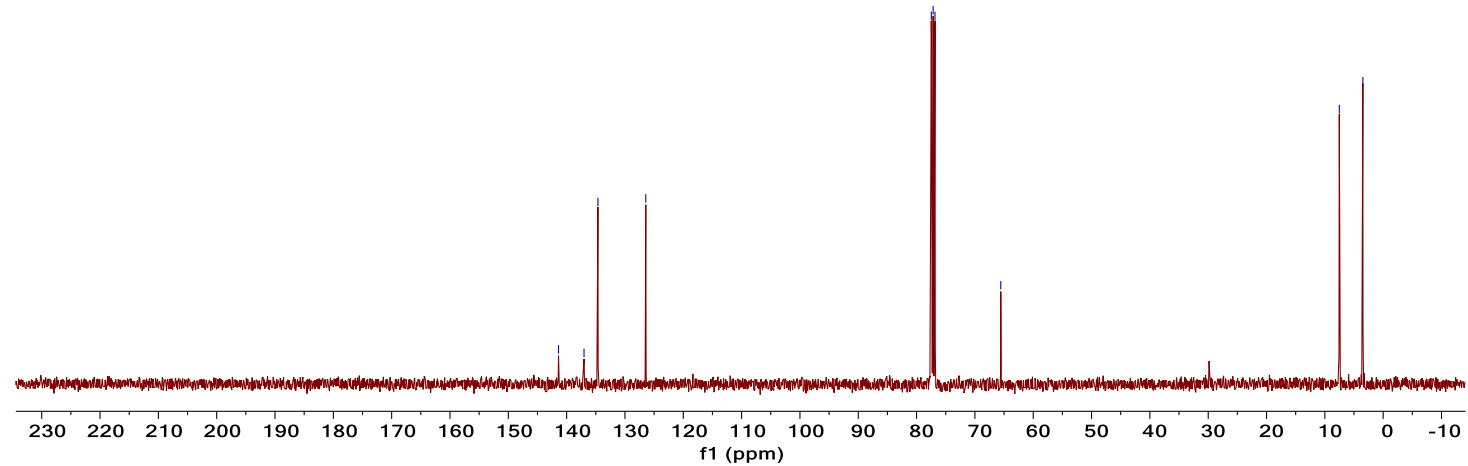


4-(4-(triethylsilyl)phenyl)morpholine (9).
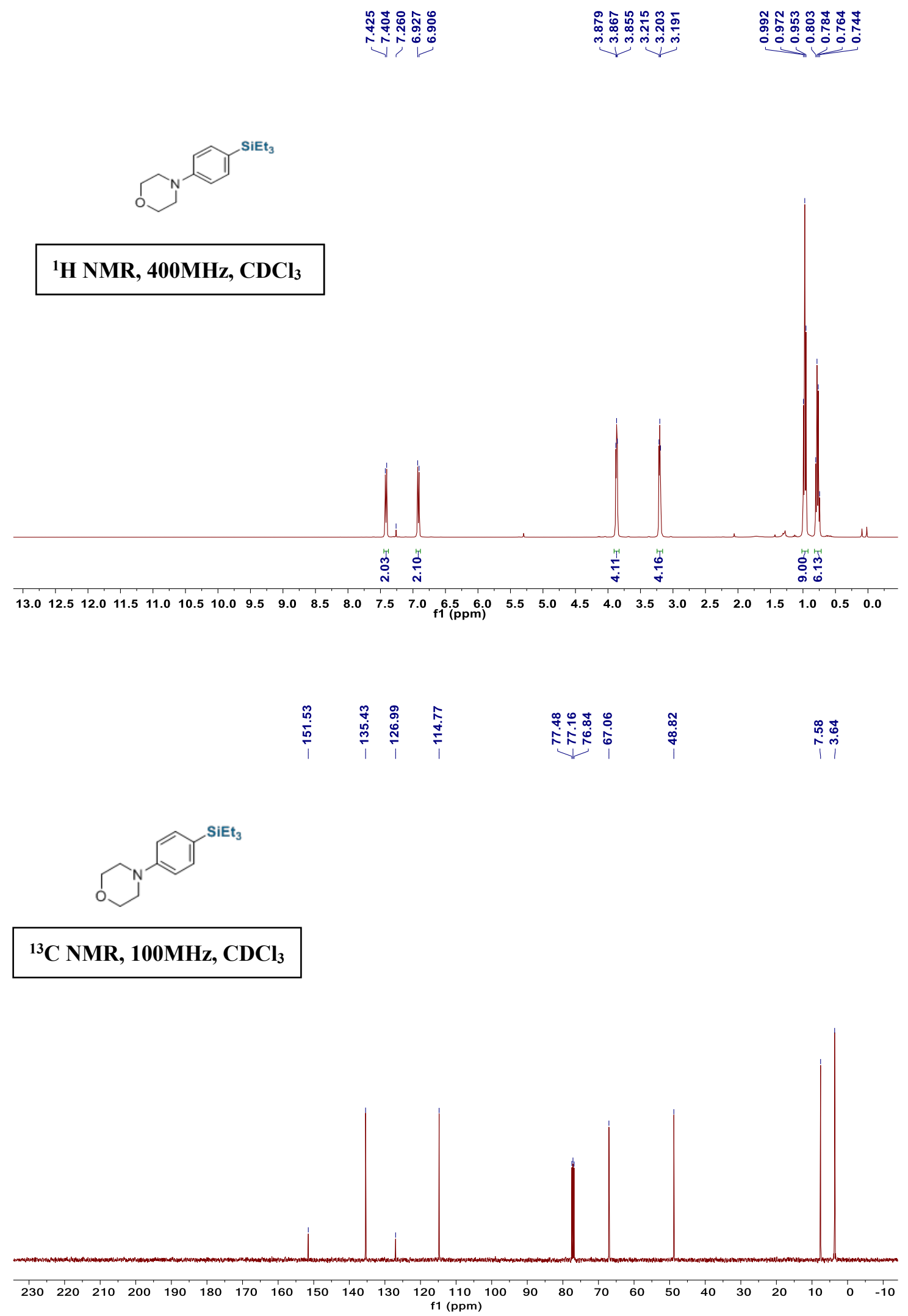
Tert-butyl methyl(4-(triethylsilyl)benzyl)carbamate (10).

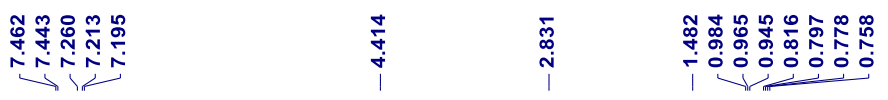

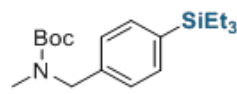

${ }^{1} \mathrm{H}$ NMR, $400 \mathrm{MHz}, \mathrm{CDCl}_{3}$

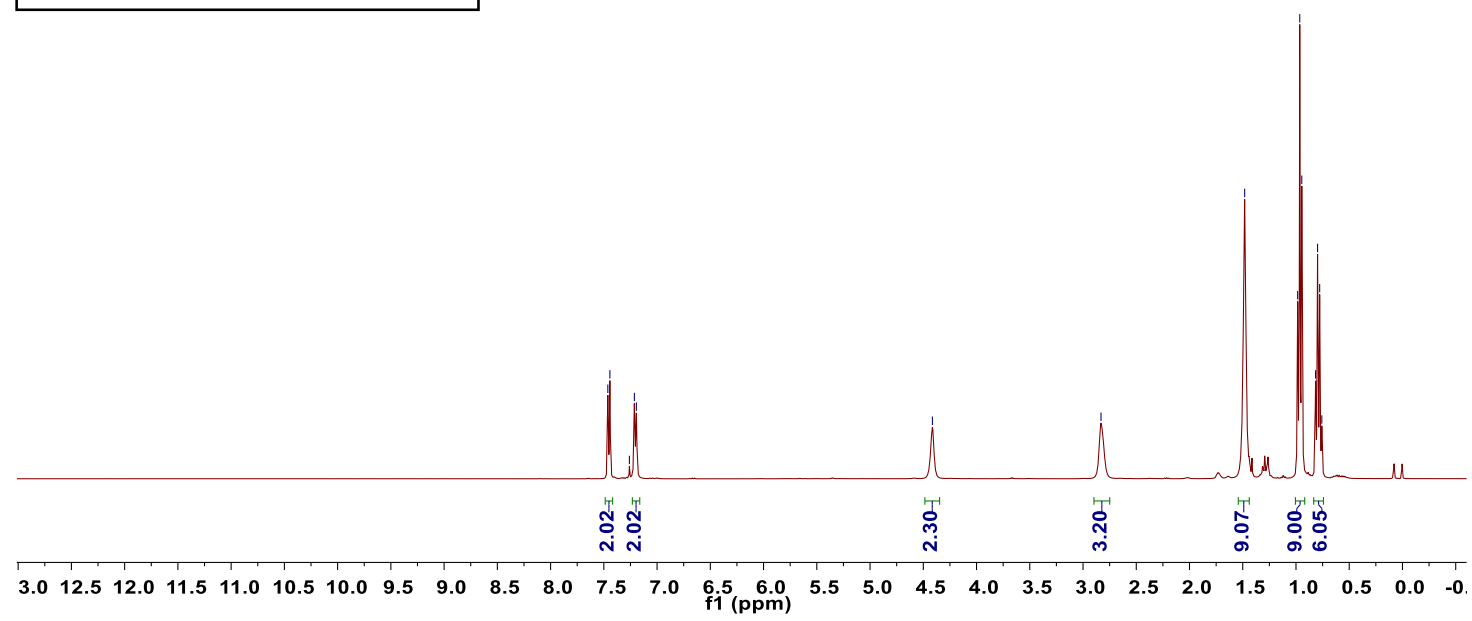

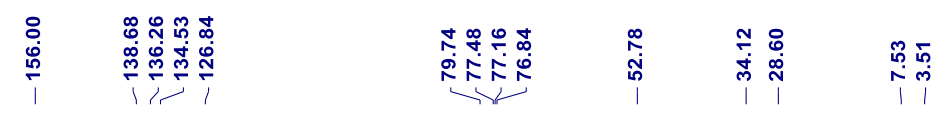

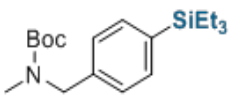

${ }^{13} \mathrm{C}$ NMR, $100 \mathrm{MHz}, \mathrm{CDCl}_{3}$

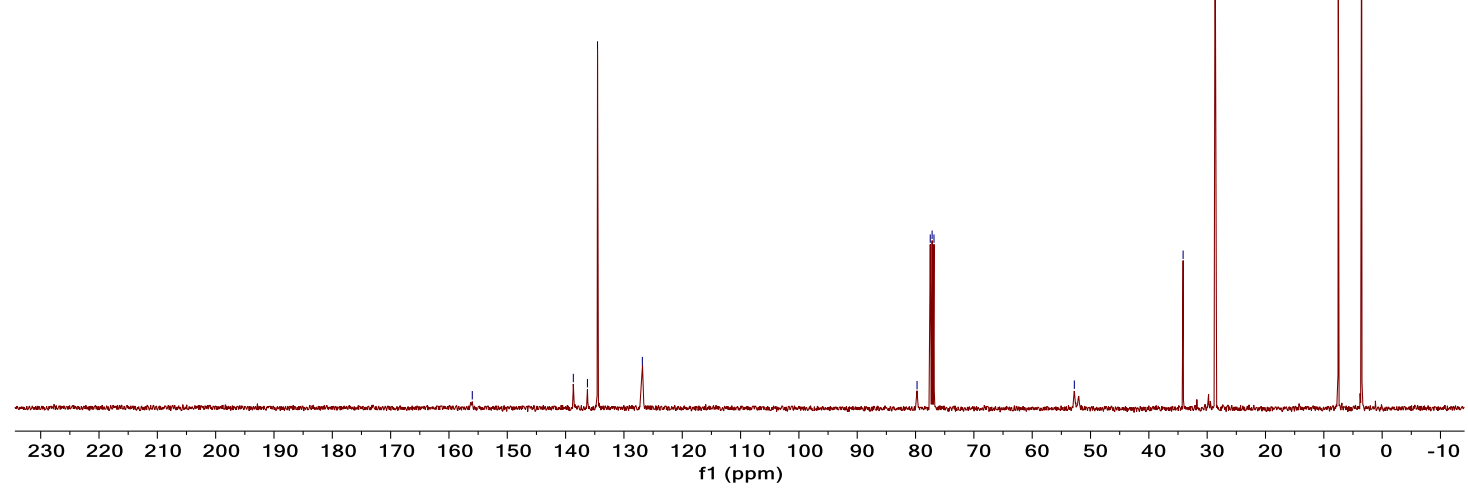


$N, N$-dimethyl-4-(triethylsilyl)aniline (11).

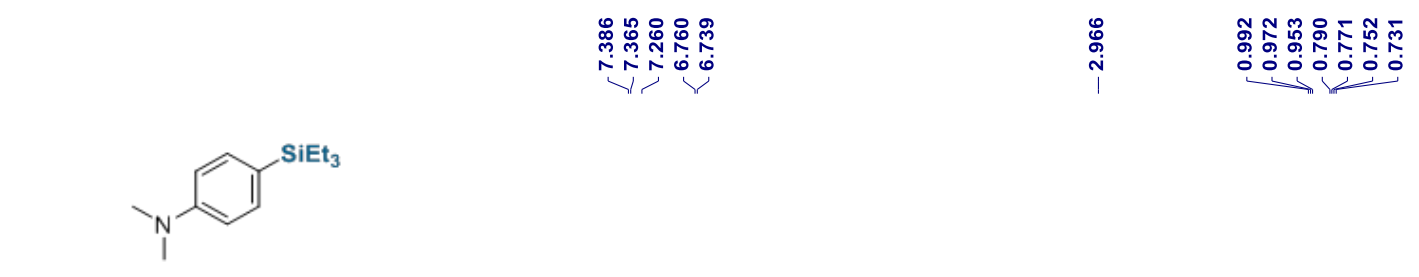

${ }^{1} \mathrm{H}$ NMR, 400MHz, $\mathrm{CDCl}_{3}$

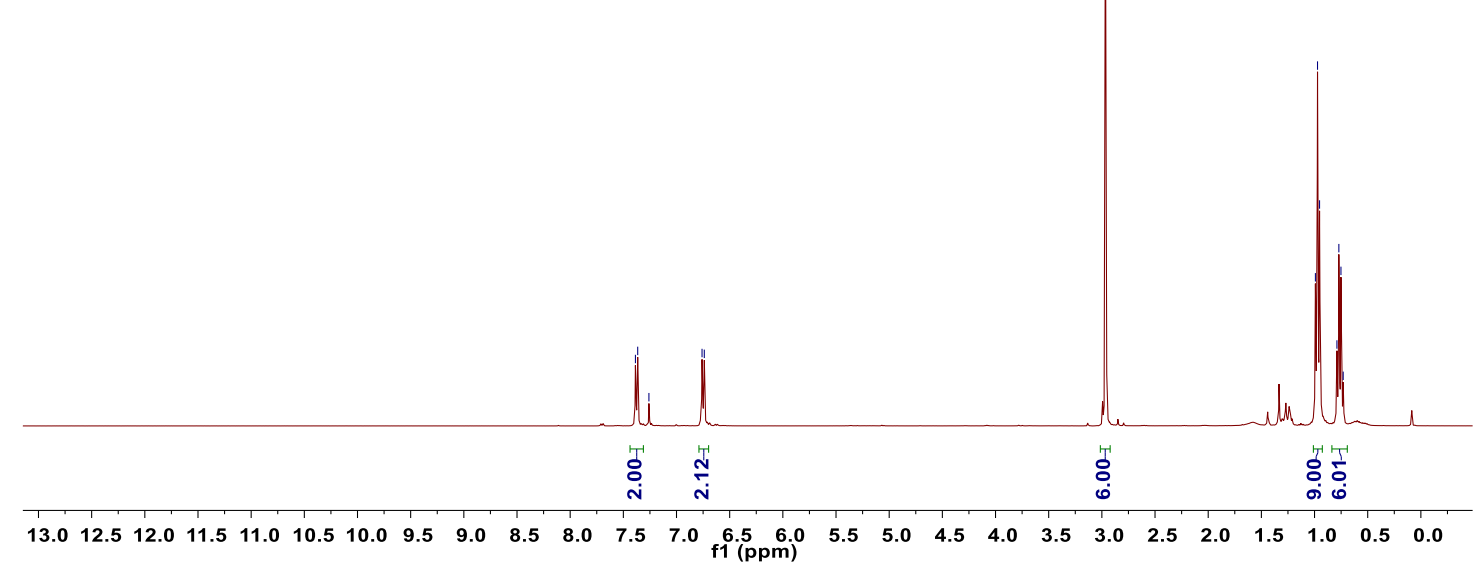

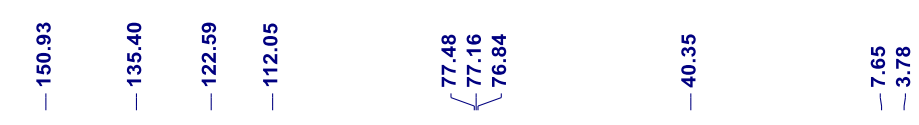

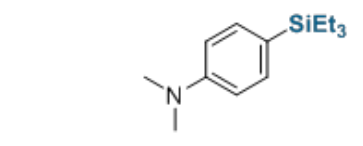

${ }^{13} \mathrm{C}$ NMR, 100MHz, $\mathrm{CDCl}_{3}$

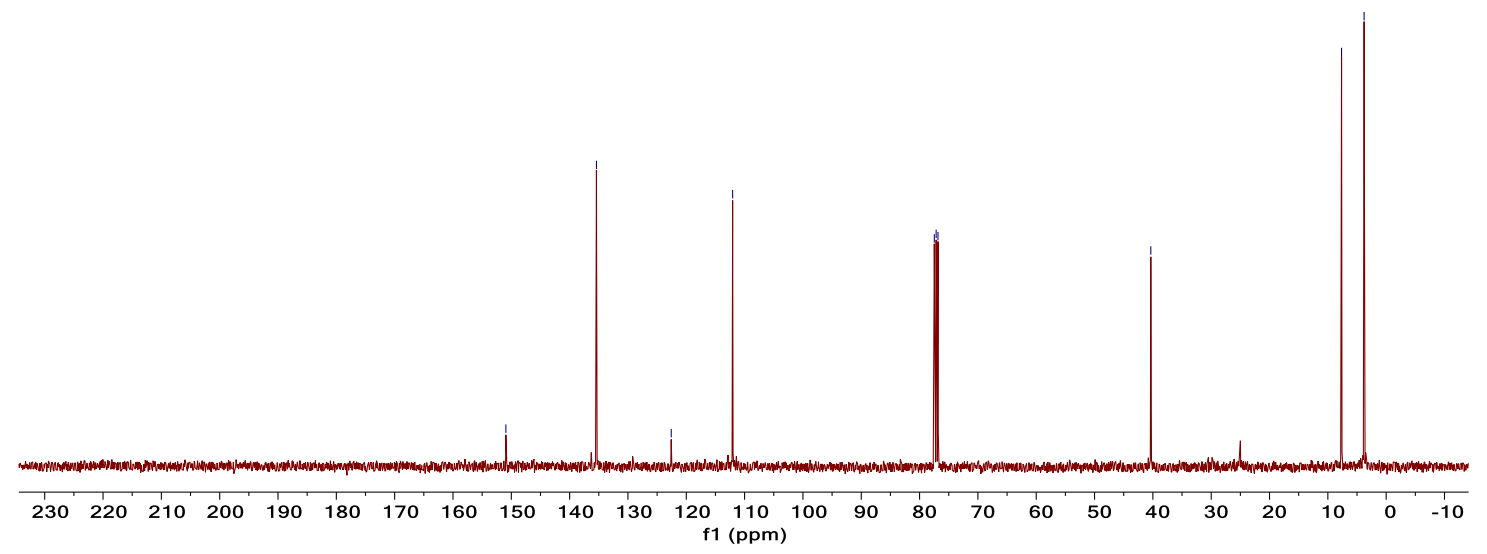


Triethyl(4-(trifluoromethyl)phenyl)silane (12).

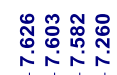

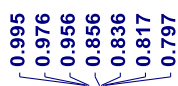

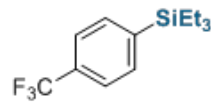

${ }^{1} \mathrm{H}$ NMR, 400MHz, $\mathrm{CDCl}_{3}$
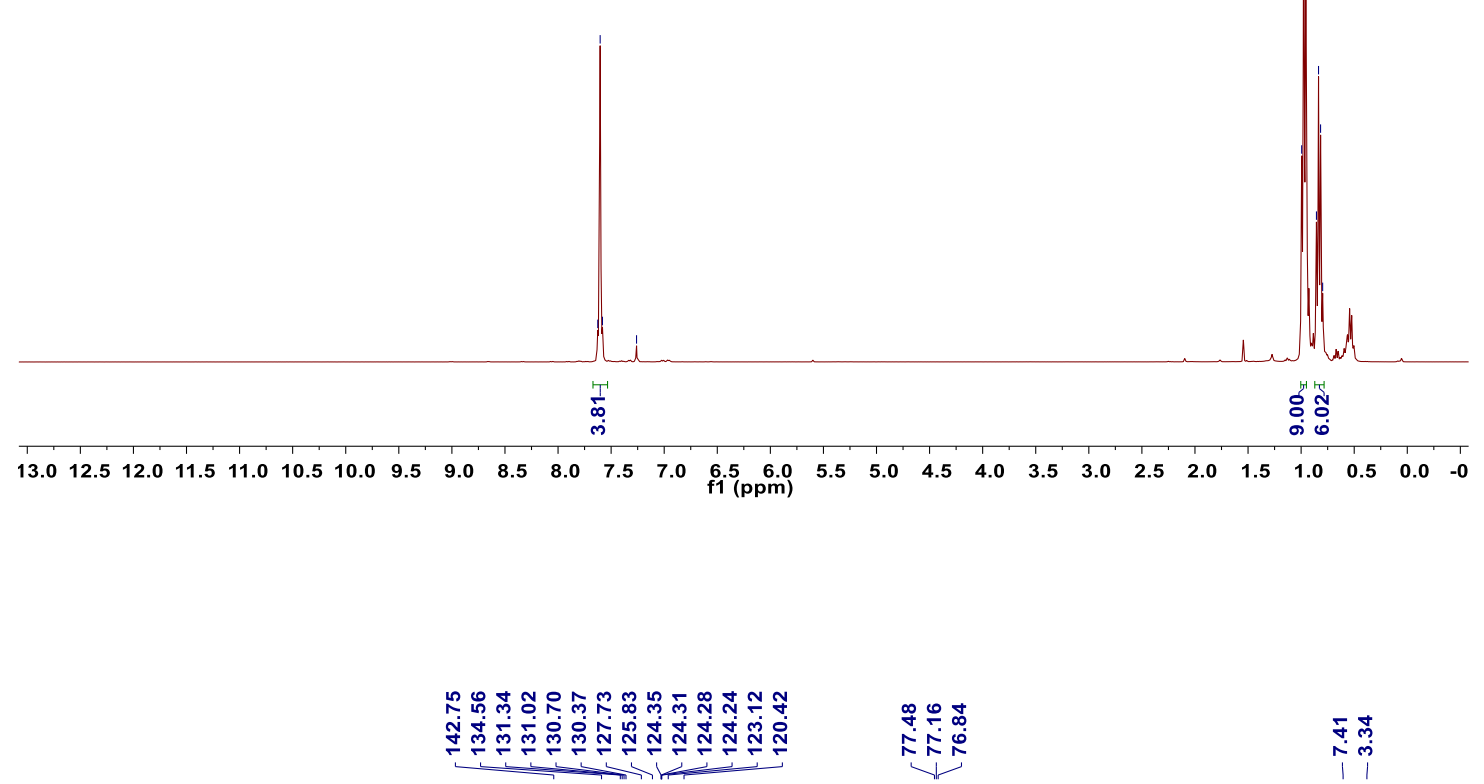

文突

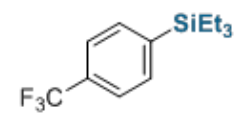

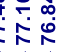

î

${ }^{13} \mathrm{C} \mathrm{NMR}, 100 \mathrm{MHz}, \mathrm{CDCl}_{3}$

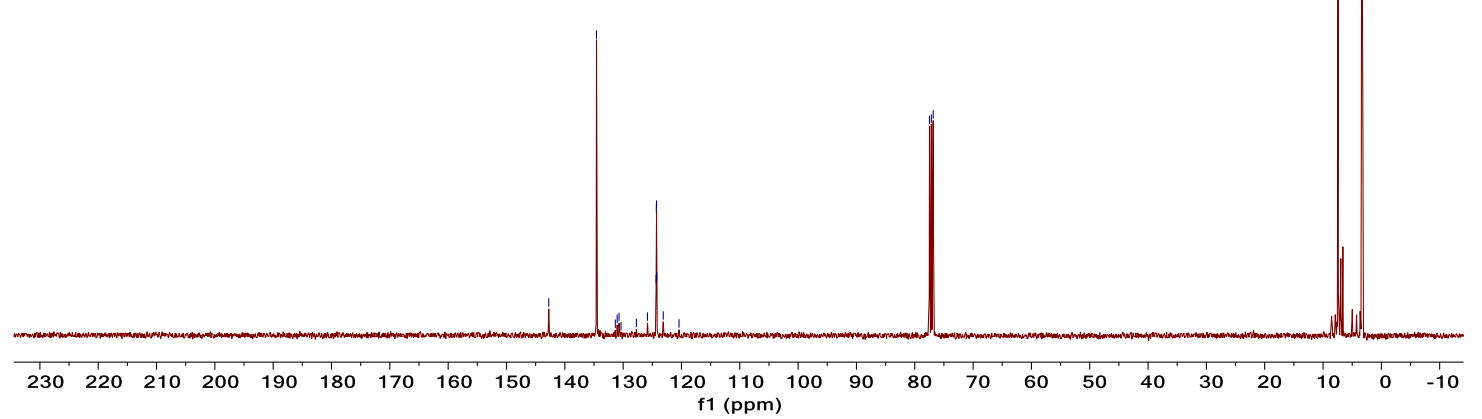


Triethyl(4-(4,4,5,5-tetramethyl-1,3-dioxolan-2-yl)phenyl)silane (13).

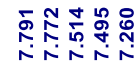

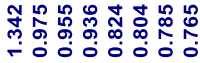

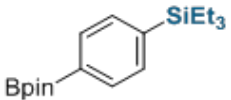

${ }^{1} \mathrm{H}$ NMR, $400 \mathrm{MHz}, \mathrm{CDCl}_{3}$
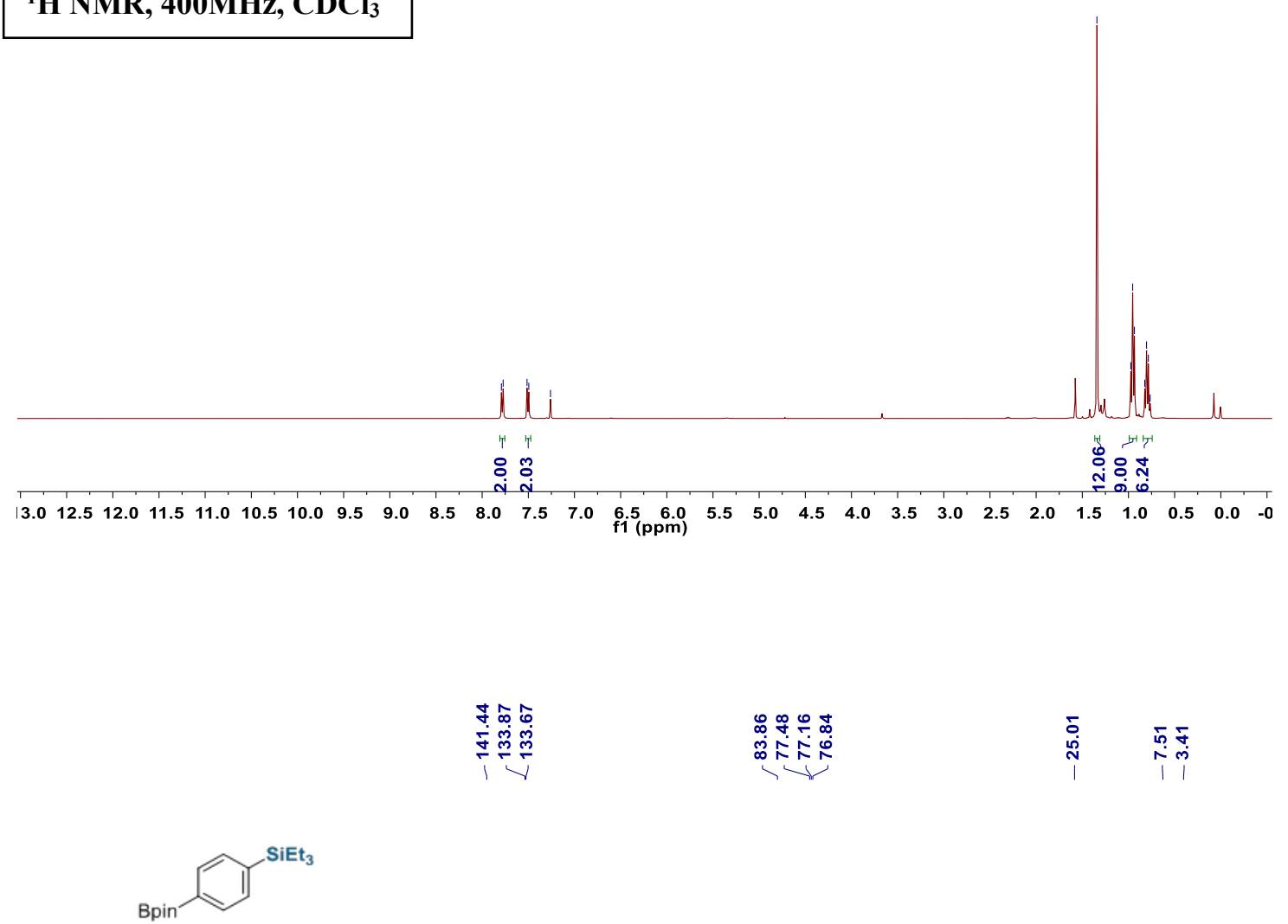

${ }^{13} \mathrm{C}$ NMR, 100MHz, $\mathrm{CDCl}_{3}$

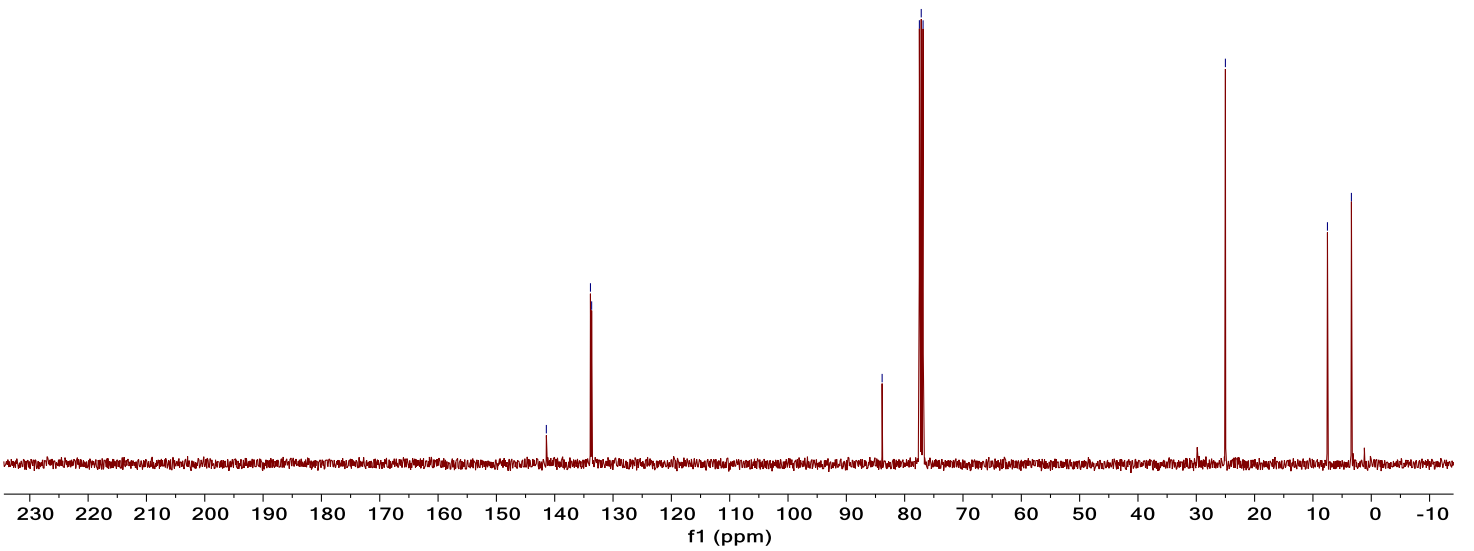


(E)-triethyl(4-styrylphenyl)silane (14).

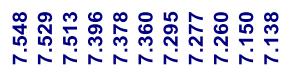

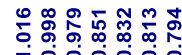

hitrkitititi

-0.00000

$\rightarrow$

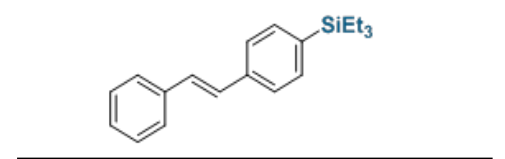

${ }^{1} \mathrm{H}$ NMR, $400 \mathrm{MHz}, \mathrm{CDCl}_{3}$
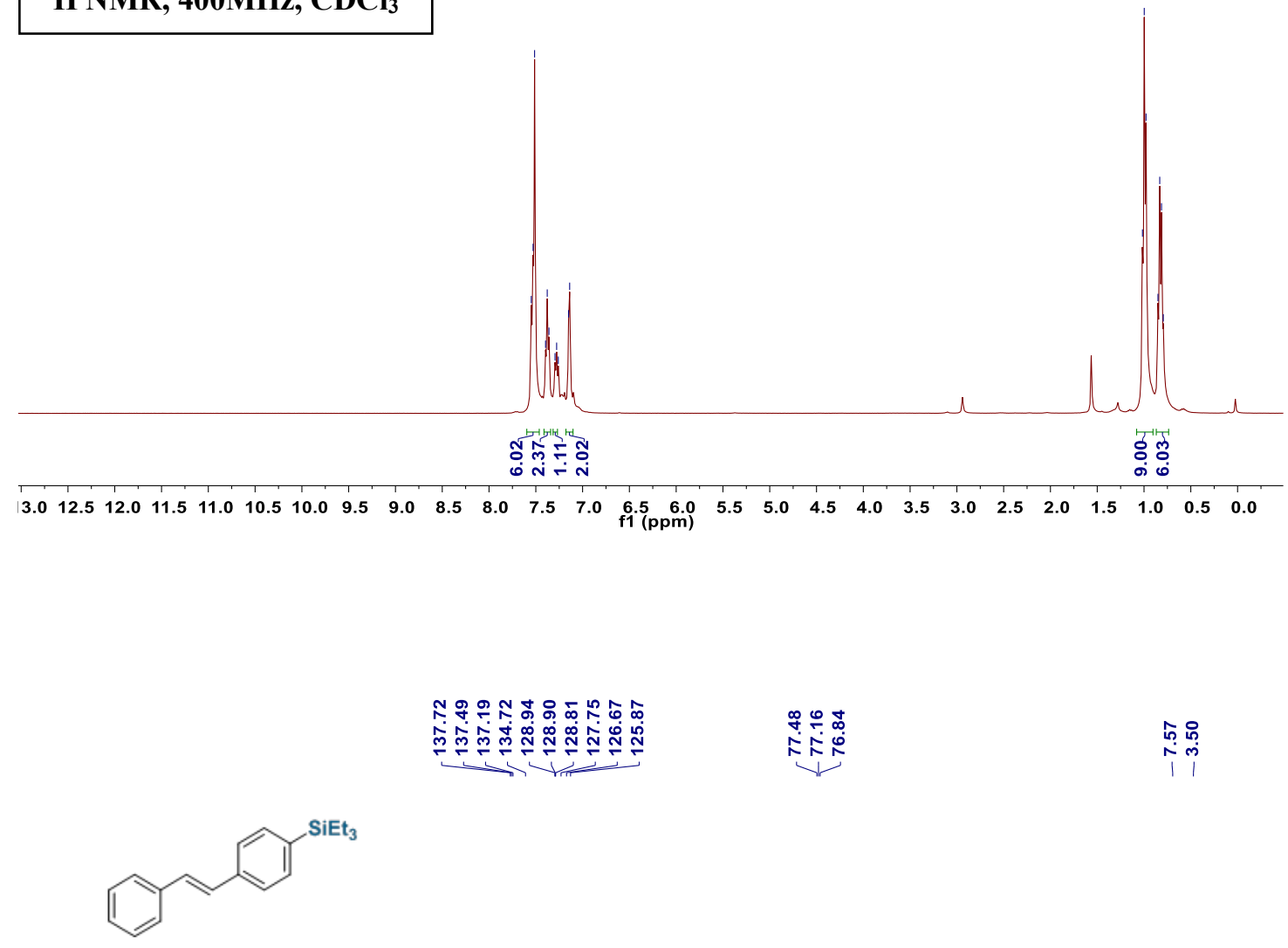

${ }^{13} \mathrm{C}$ NMR, $100 \mathrm{MHz}, \mathrm{CDCl}_{3}$

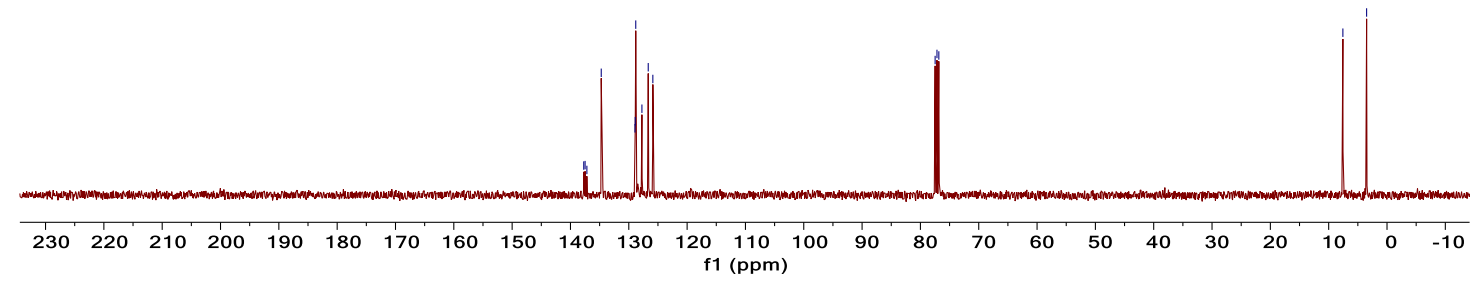


[1,1'-biphenyl]-4-yltriethylsilane (15).

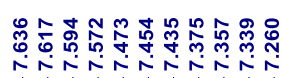

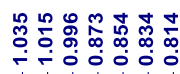

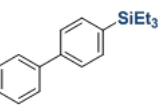

${ }^{1} \mathrm{H}$ NMR, $400 \mathrm{MHz}, \mathrm{CDCl}_{3}$

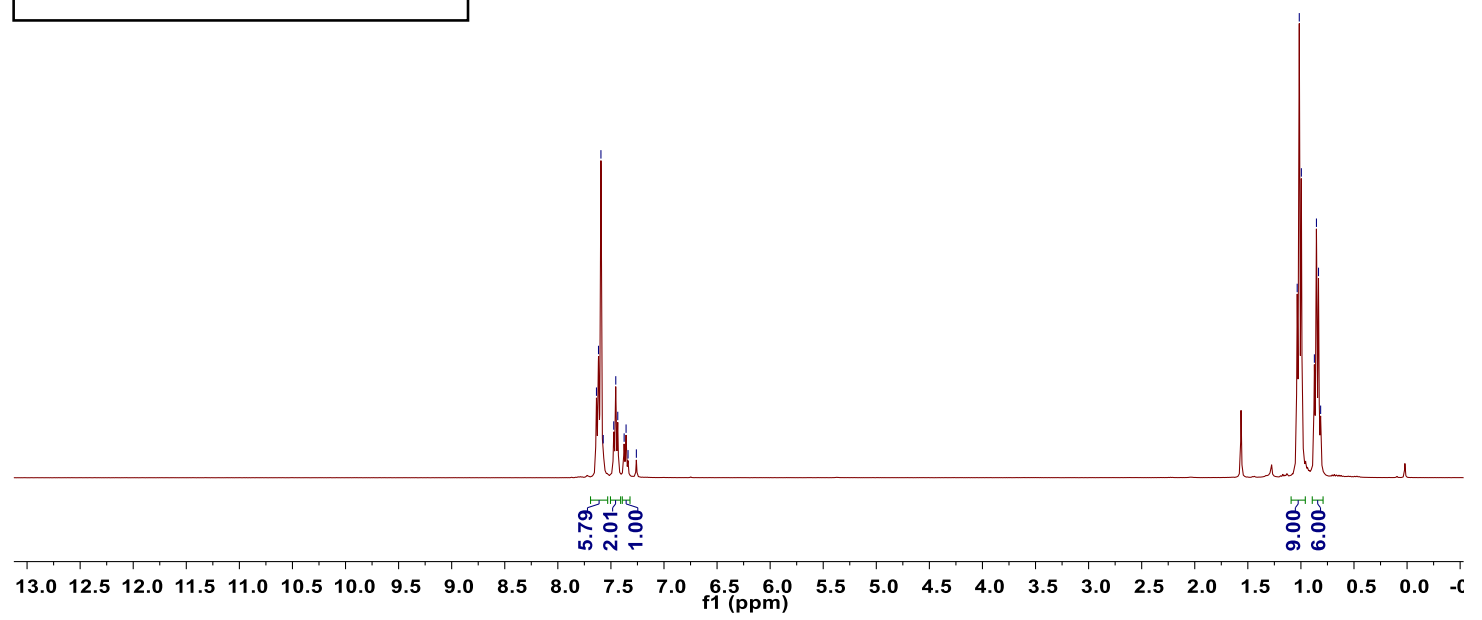

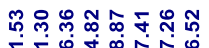

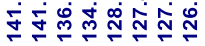

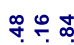

年正

承

ii

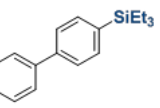

${ }^{13} \mathrm{C}$ NMR, $100 \mathrm{MHz}, \mathrm{CDCl}_{3}$

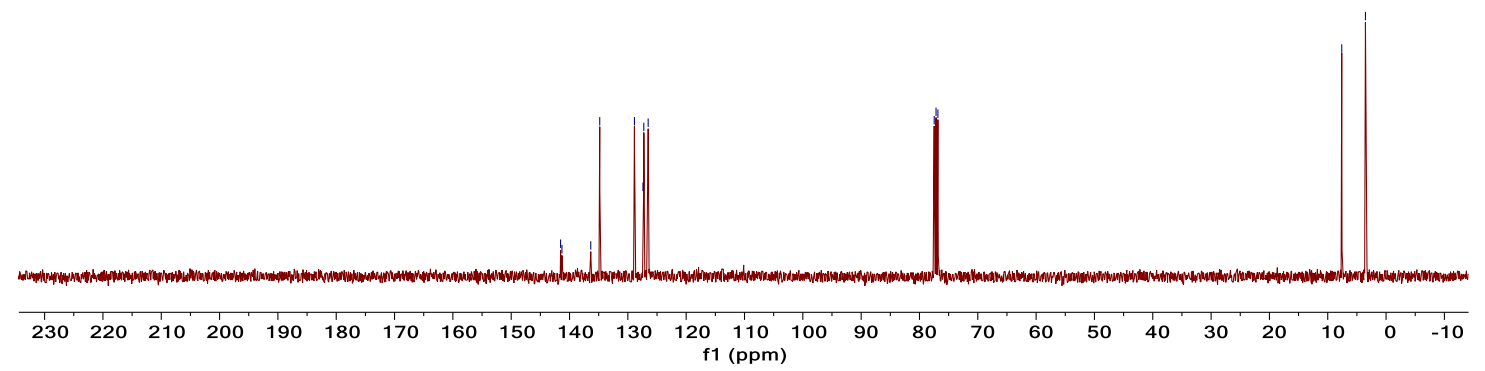


Triethyl(4'-methoxy-[1,1'-biphenyl]-4-yl)silane (16).

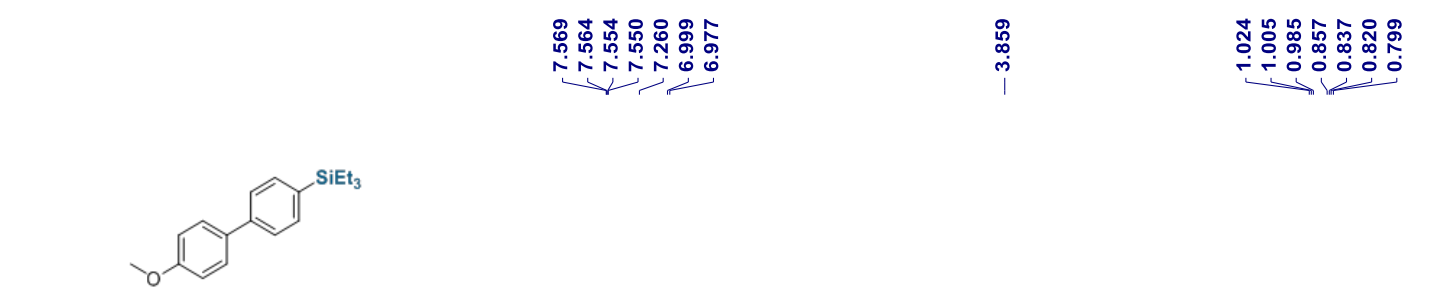

${ }^{1} \mathrm{H}$ NMR, $400 \mathrm{MHz}, \mathrm{CDCl}_{3}$

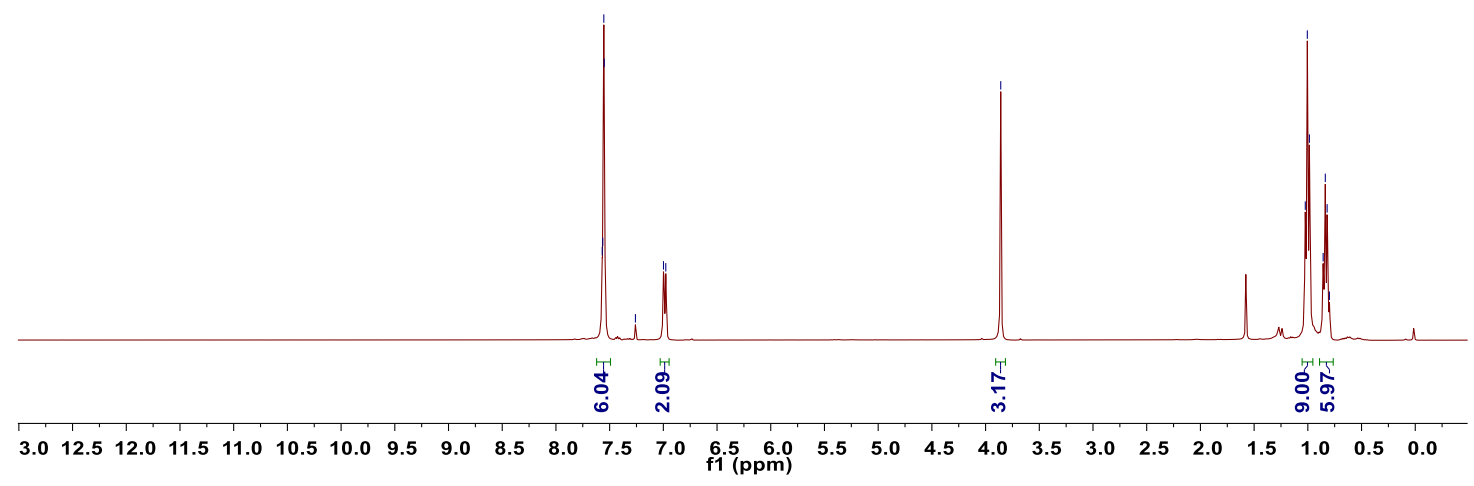

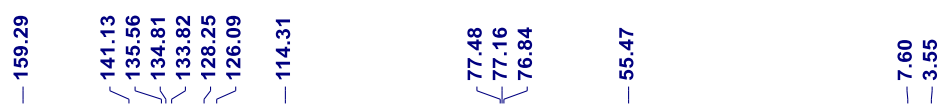

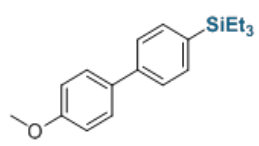

${ }^{13} \mathrm{C}$ NMR, 100MHz, $\mathrm{CDCl}_{3}$

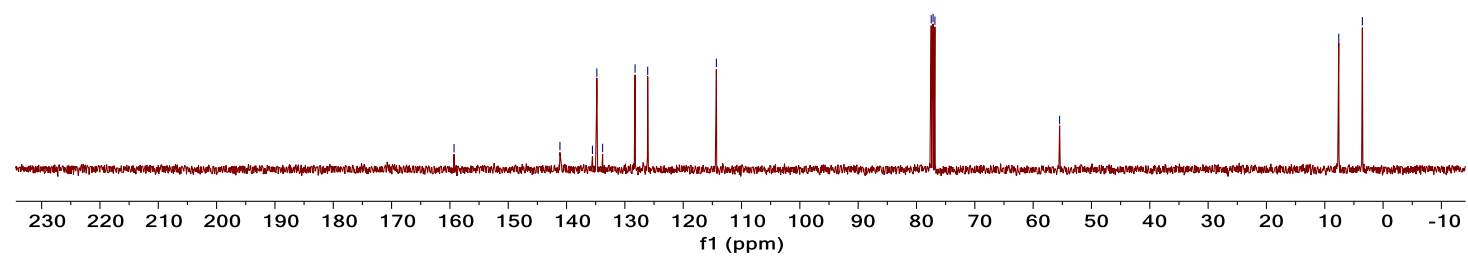


(4'-(tert-butyl)-[1,1'-biphenyl]-4-yl)triethylsilane (17).
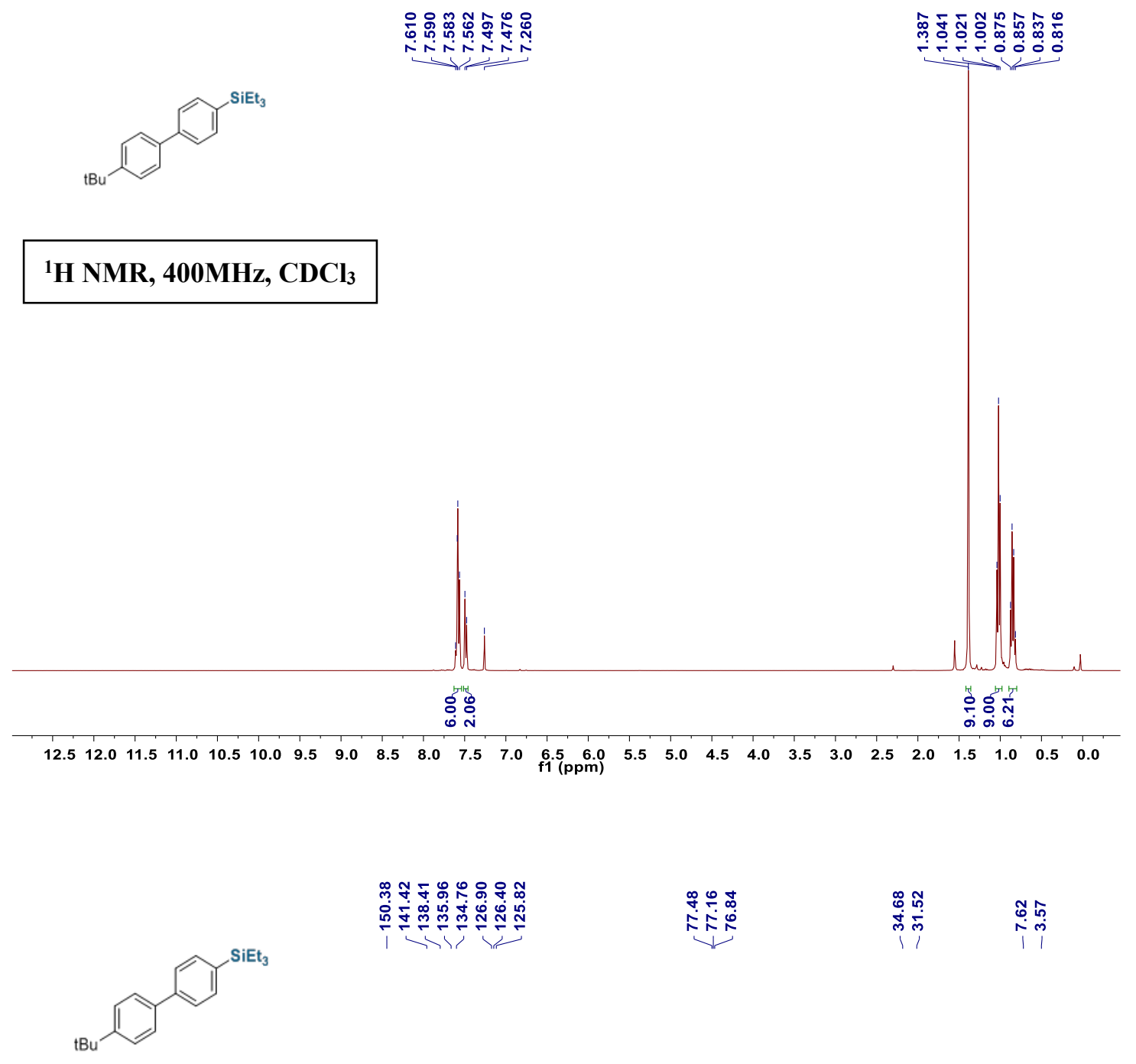

${ }^{13} \mathrm{C}$ NMR, $100 \mathrm{MHz}, \mathrm{CDCl}_{3}$

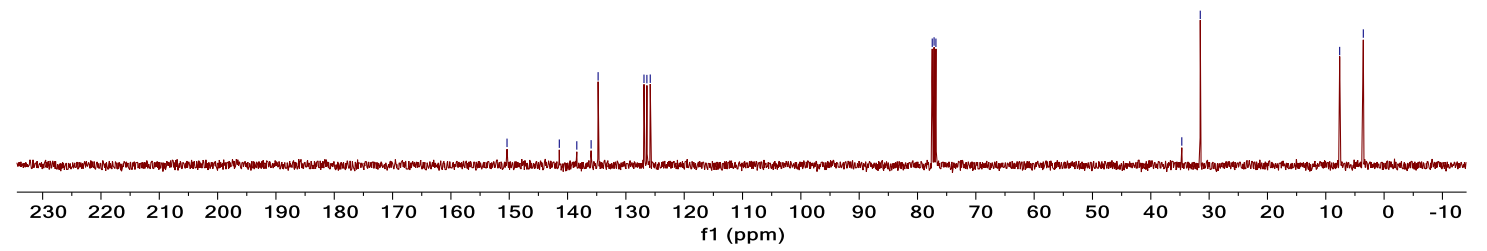


(4-(benzo[d][1,3]dioxol-5-yl)phenyl)triethylsilane (18).

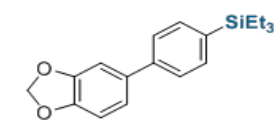

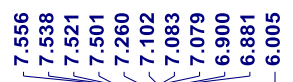

${ }^{1} \mathrm{H}$ NMR, $400 \mathrm{MHz}, \mathrm{CDCl}_{3}$
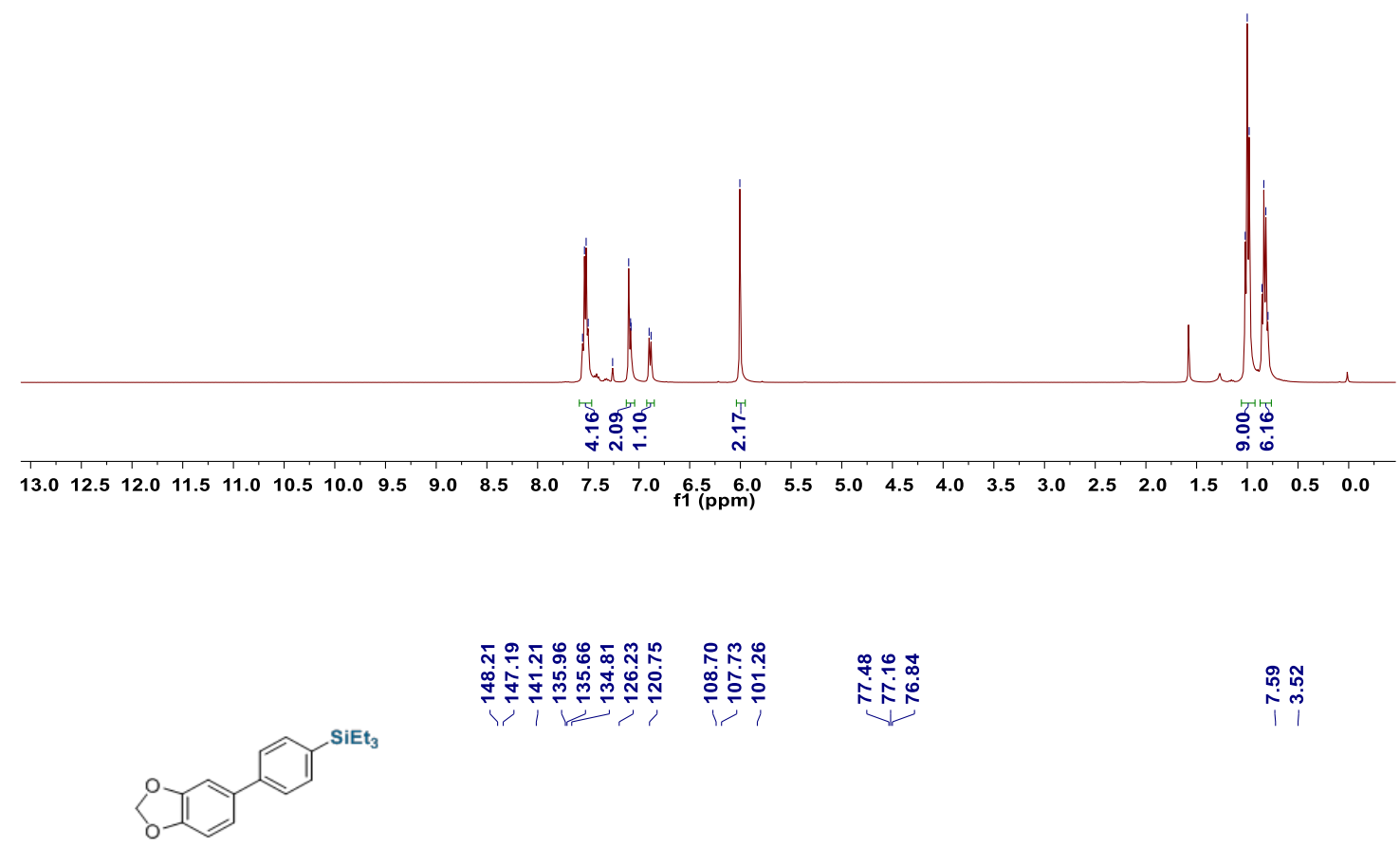

${ }^{13} \mathrm{C}$ NMR, $100 \mathrm{MHz}, \mathrm{CDCl}_{3}$

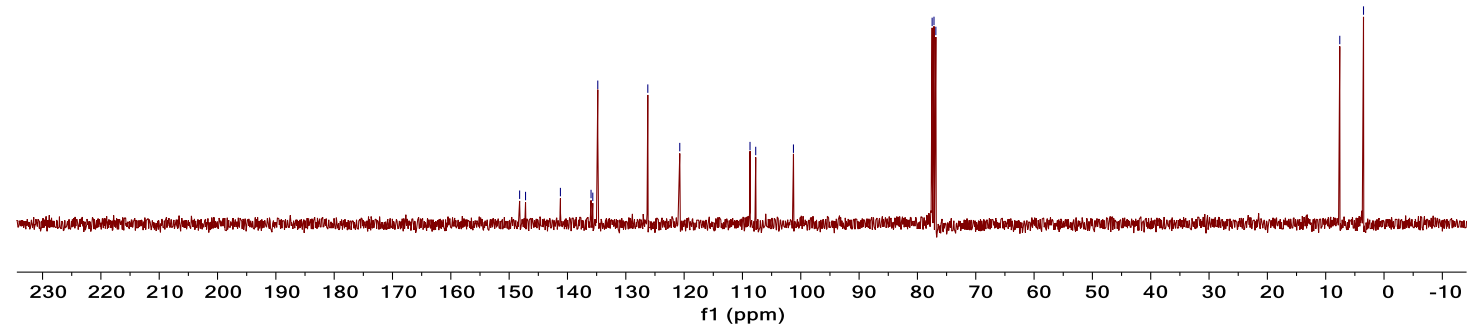


Triethyl(4'-phenoxy-[1,1'-biphenyl]-4-yl)silane (19).

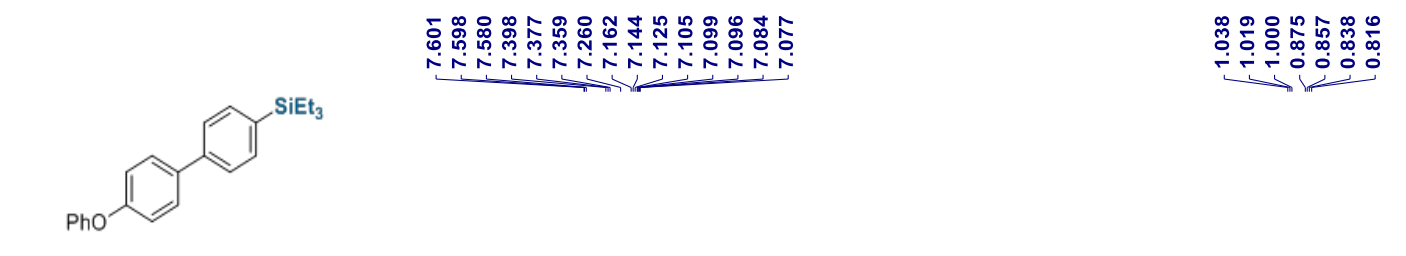

${ }^{1} \mathrm{H}$ NMR, 400MHz, $\mathrm{CDCl}_{3}$

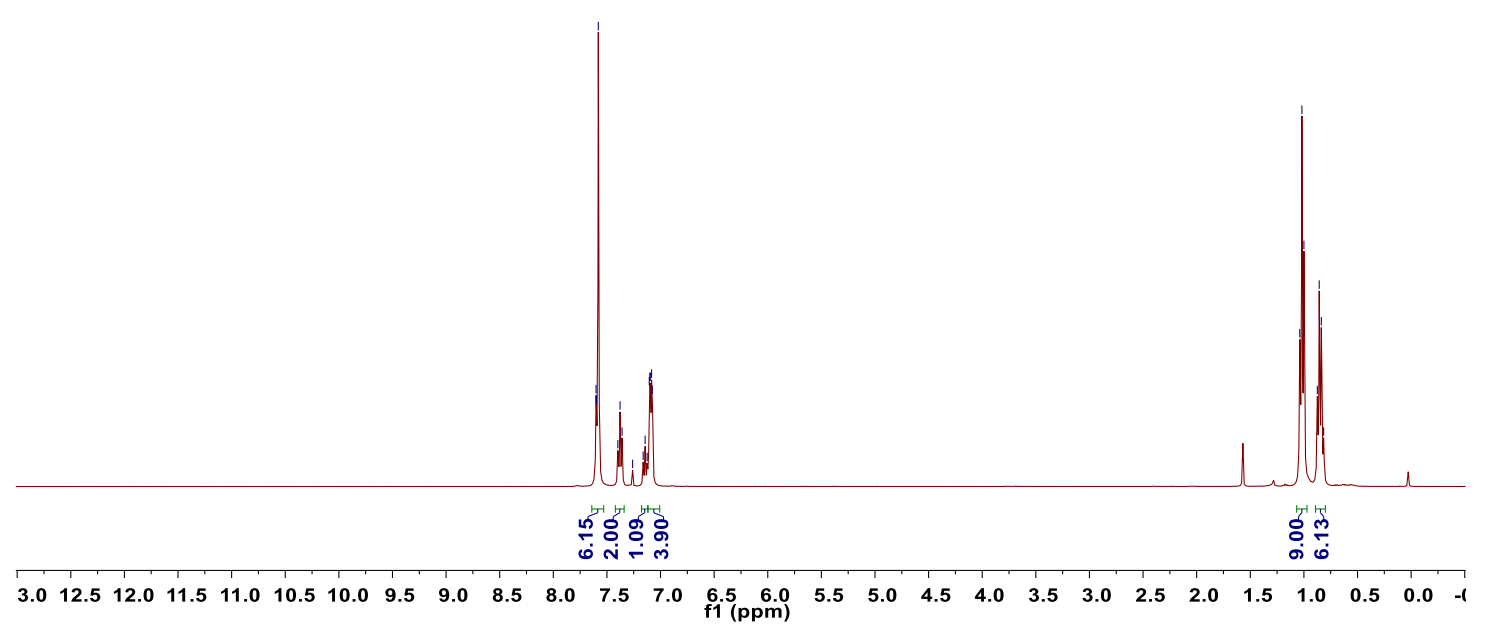

พ⿻ำ 뭉ำ

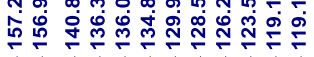

$\stackrel{\infty}{\stackrel{\infty}{i}}$

(

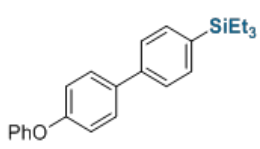

${ }^{13} \mathrm{C}$ NMR, $100 \mathrm{MHz}, \mathrm{CDCl}_{3}$

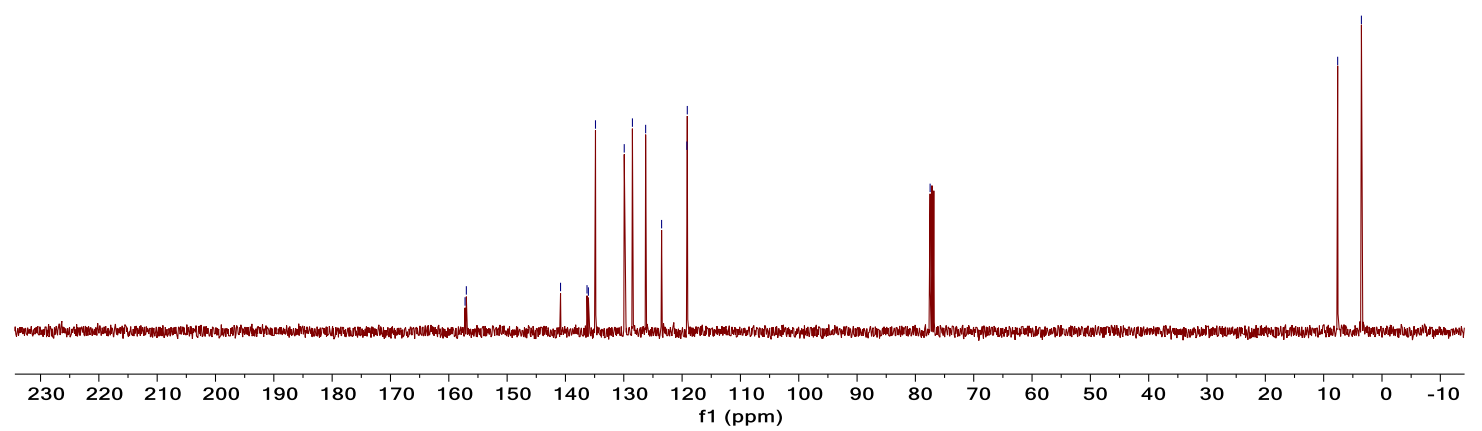


Triethyl(4-(naphthalen-2-yl)phenyl)silane (20).
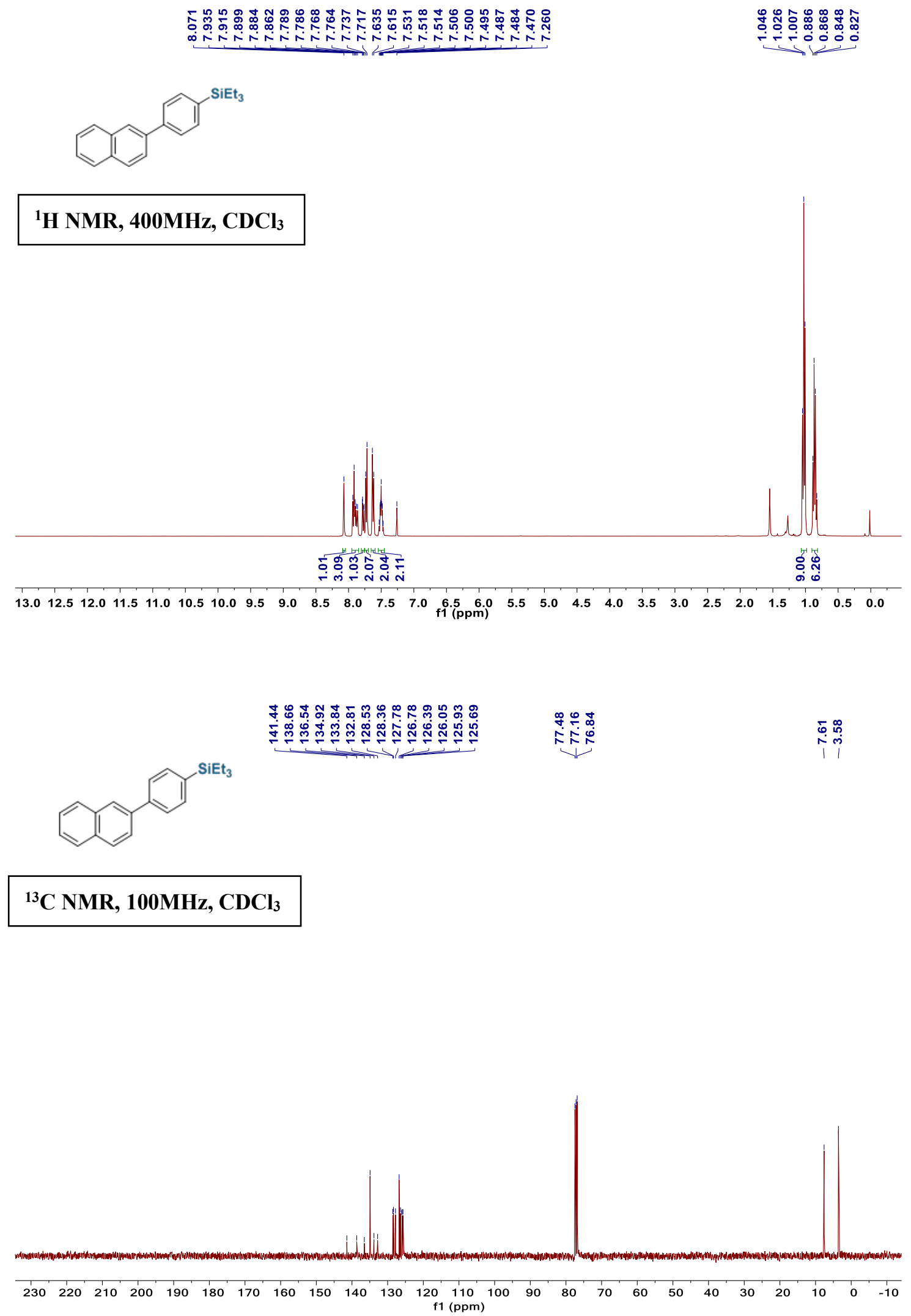
Triethyl(4'-isopropoxy-[1,1'-biphenyl]-4-yl)silane (21).

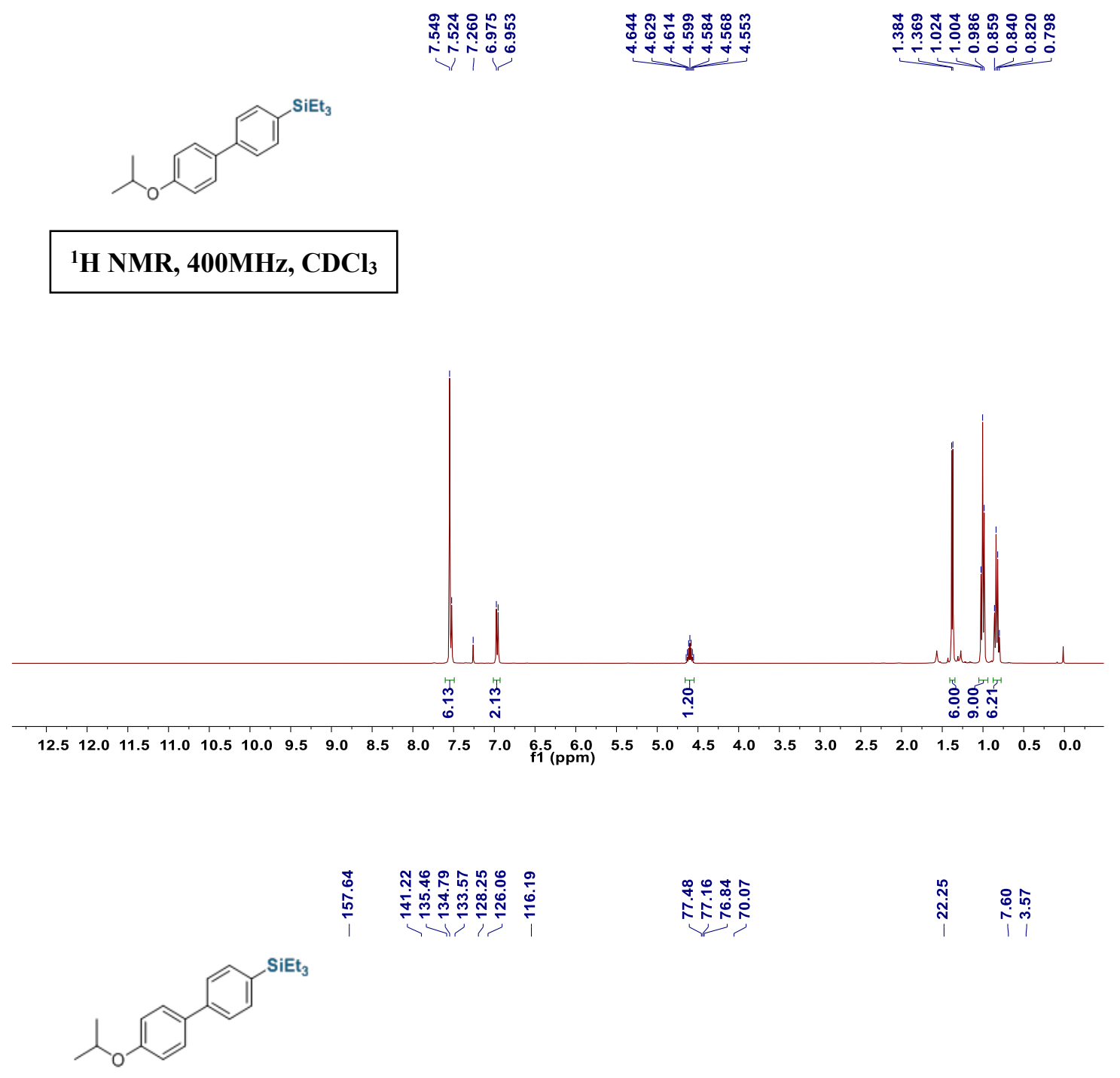

${ }^{13} \mathrm{C}$ NMR, $100 \mathrm{MHz}, \mathrm{CDCl}_{3}$

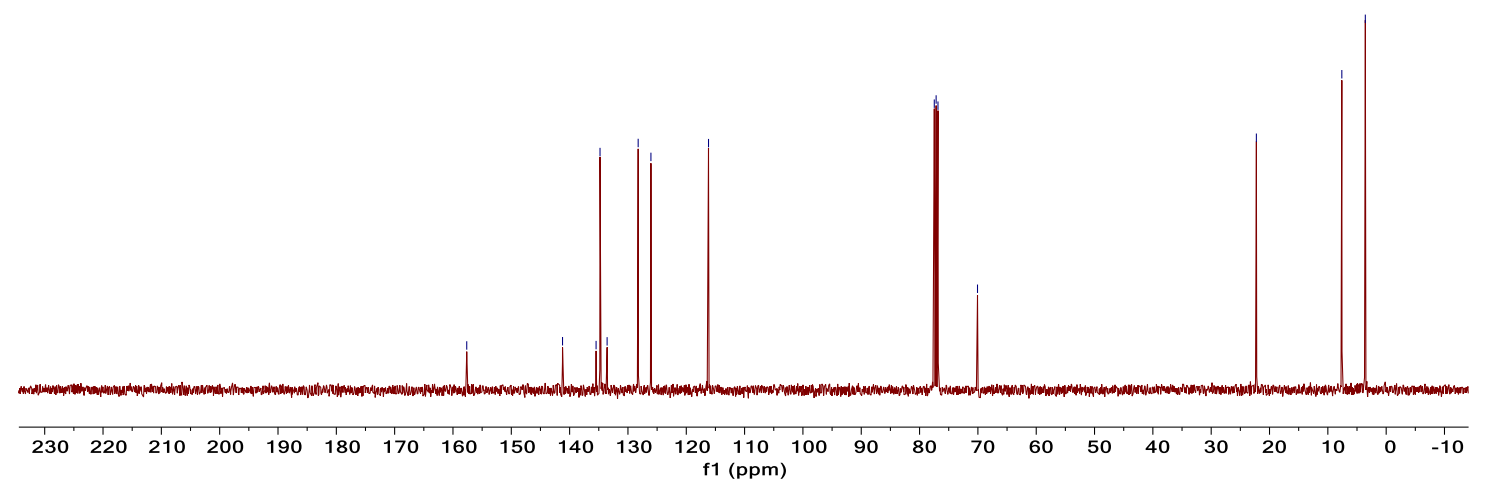


Triethyl(4'-(trifluoromethyl)-[1,1'-biphenyl]-4-yl)silane (22).

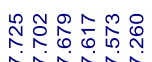

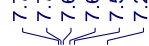

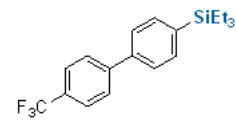

${ }^{1} \mathrm{H}$ NMR, $400 \mathrm{MHz}, \mathrm{CDCl}_{3}$

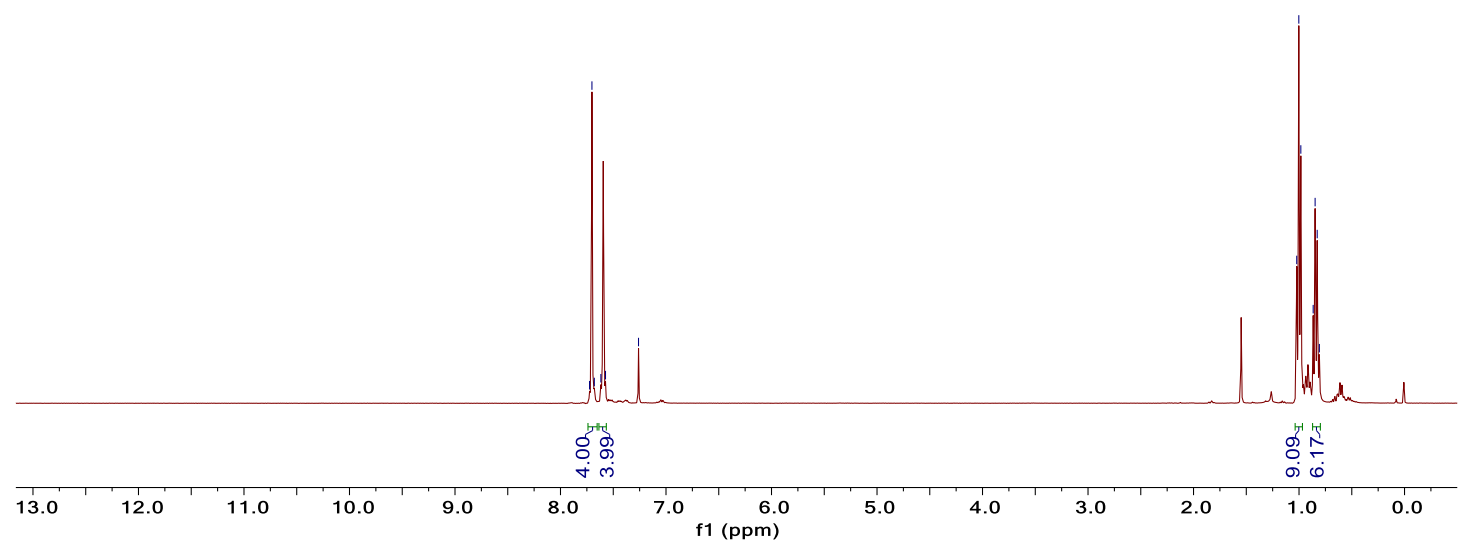

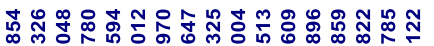

ن jo

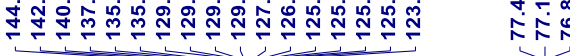

员

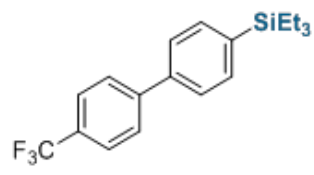

${ }^{13} \mathrm{C}$ NMR, $100 \mathrm{MHz}, \mathrm{CDCl}_{3}$

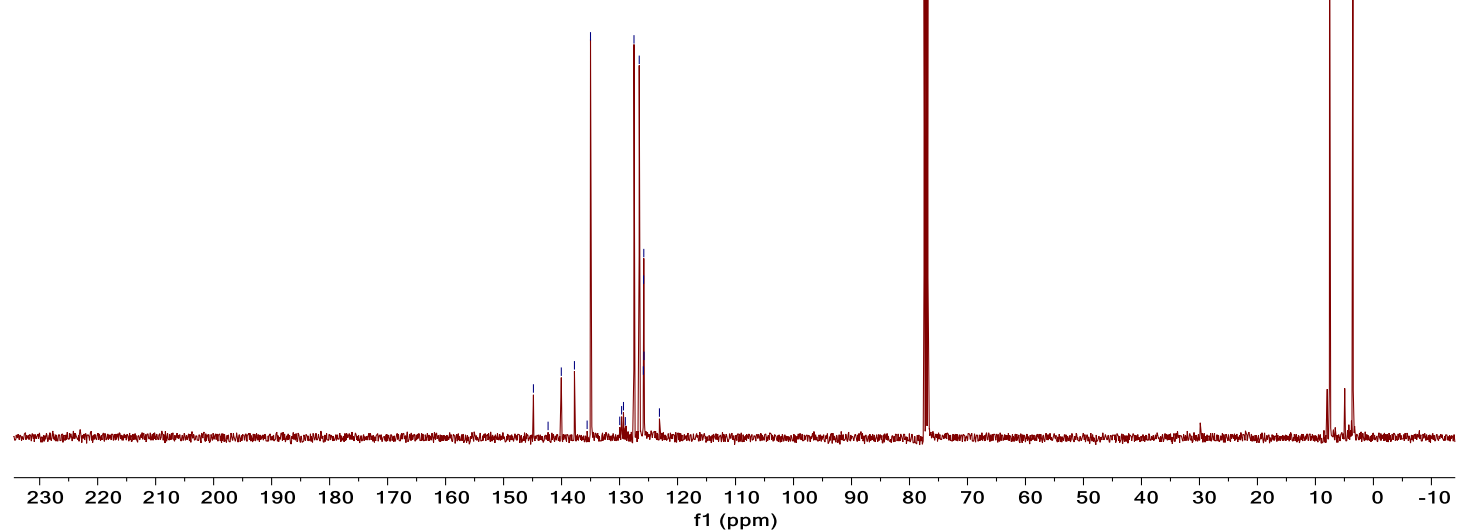


3-(4-(triethylsilyl)phenyl)pyridine (23).

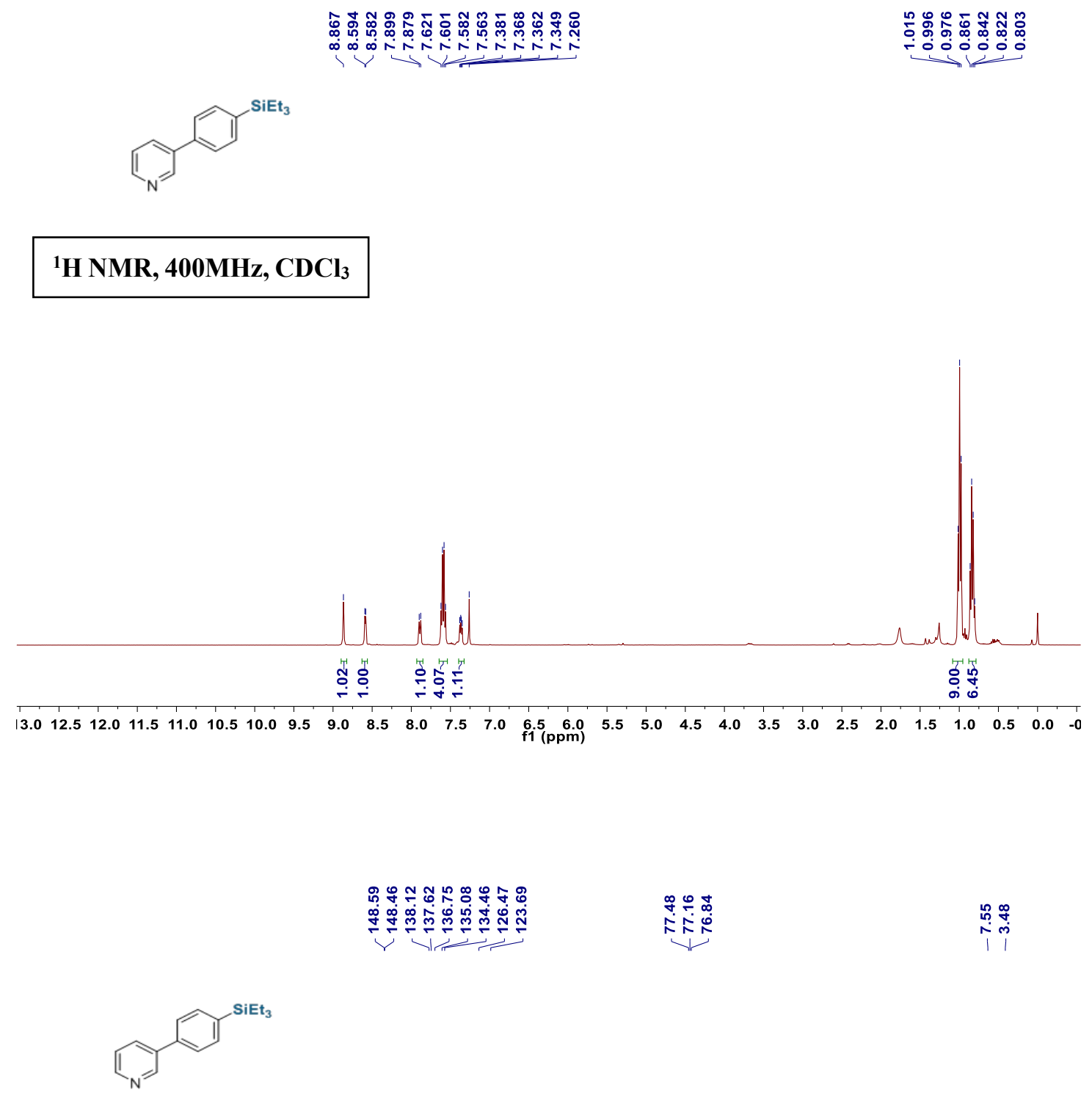

${ }^{13} \mathrm{C}$ NMR, $100 \mathrm{MHz}, \mathrm{CDCl}_{3}$

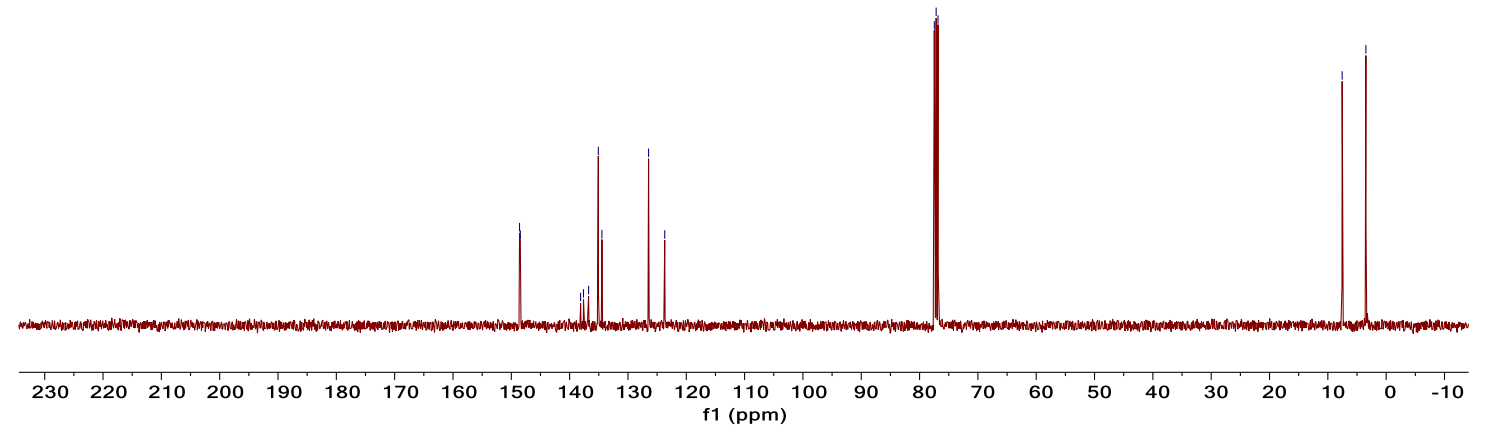


[1,1'-biphenyl]-2-yltriethylsilane (24).

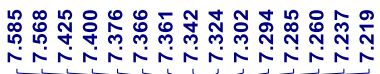

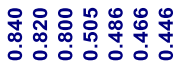

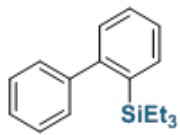

${ }^{1} \mathrm{H}$ NMR, $100 \mathrm{MHz}, \mathrm{CDCl}_{3}$

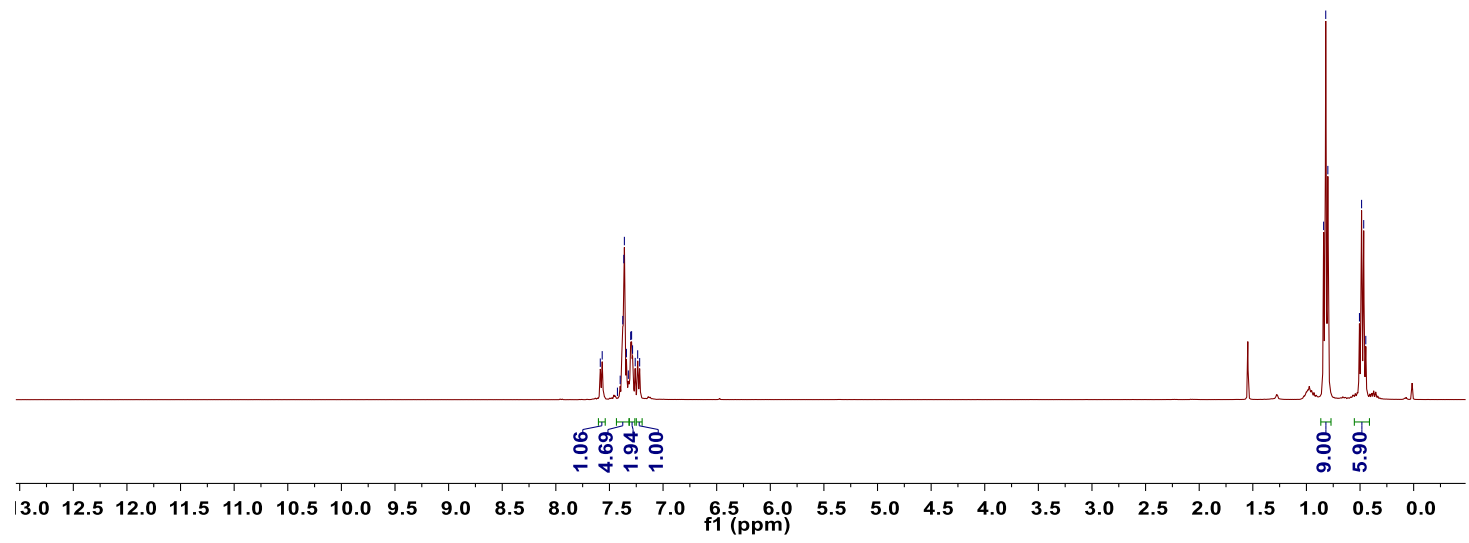

एक

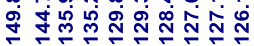

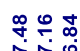

等占

苍 $\stackrel{0}{+}$

i广

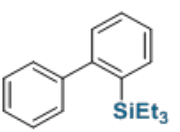

${ }^{13} \mathrm{C}$ NMR, $100 \mathrm{MHz}, \mathrm{CDCl}_{3}$

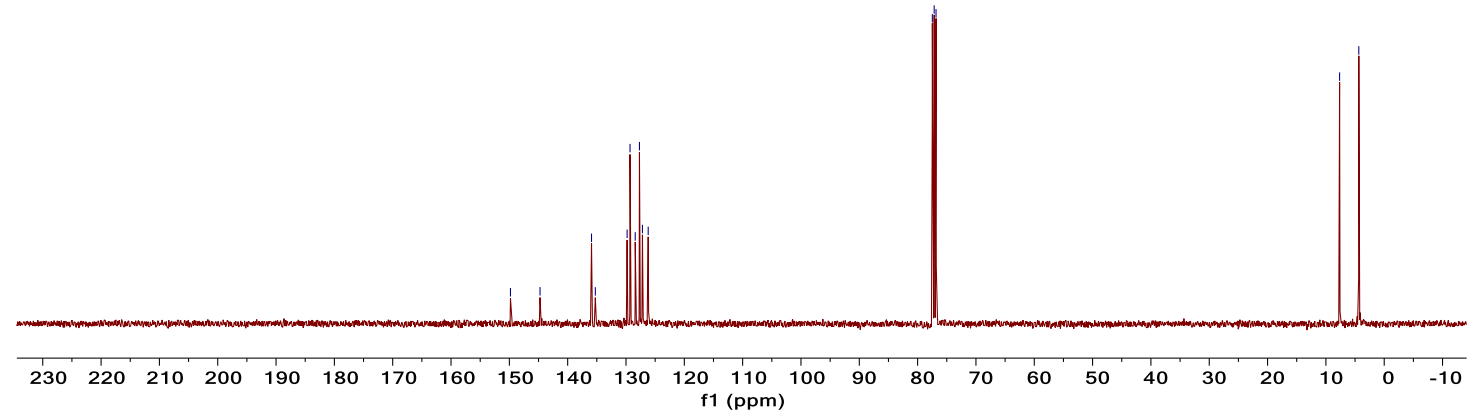


2-methoxy-5-(triethylsilyl)pyridine (25).

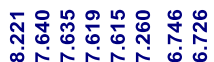

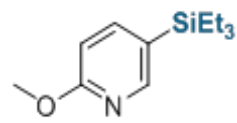

${ }^{1} \mathrm{H}$ NMR, $400 \mathrm{MHz}, \mathrm{CDCl}_{3}$

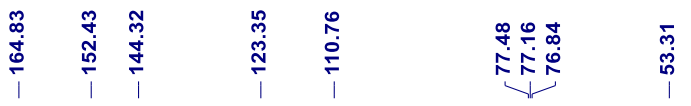

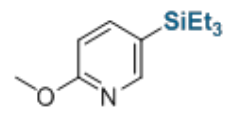

${ }^{13} \mathrm{C}$ NMR, 100MHz, $\mathrm{CDCl}_{3}$

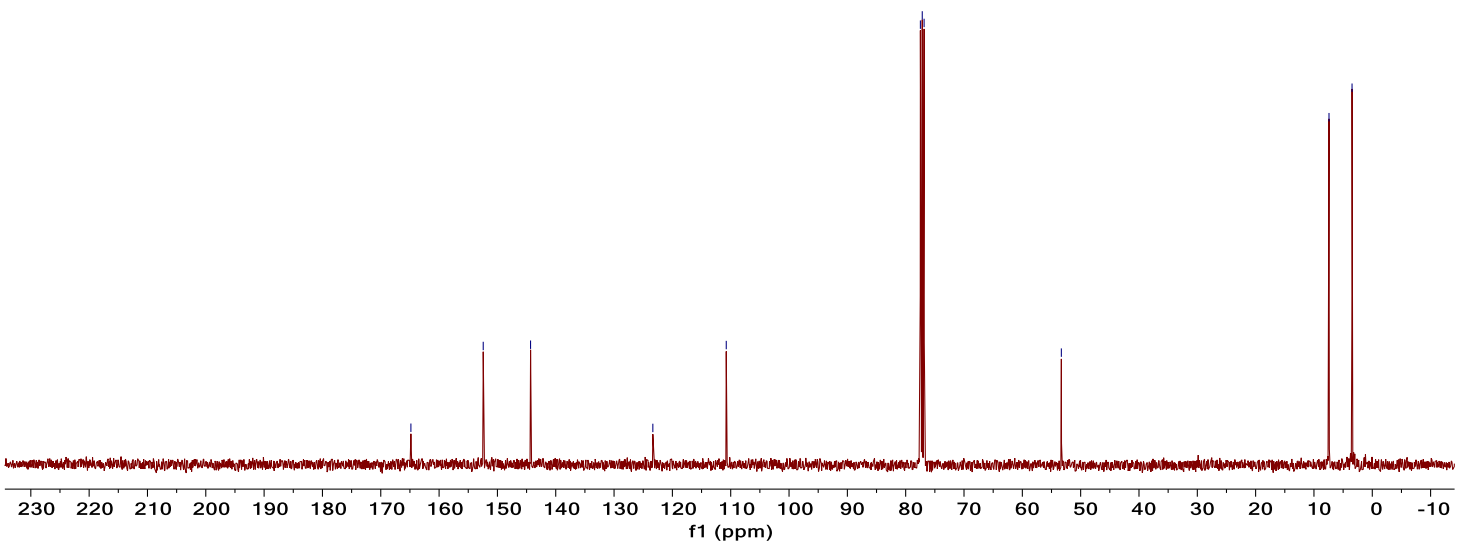


3-(triethylsilyl)pyridine (26).

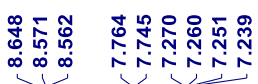

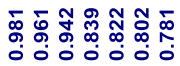

$\mathrm{SiEt}_{\mathrm{N}}$

${ }^{1} \mathrm{H}$ NMR, 400MHz, $\mathrm{CDCl}_{3}$

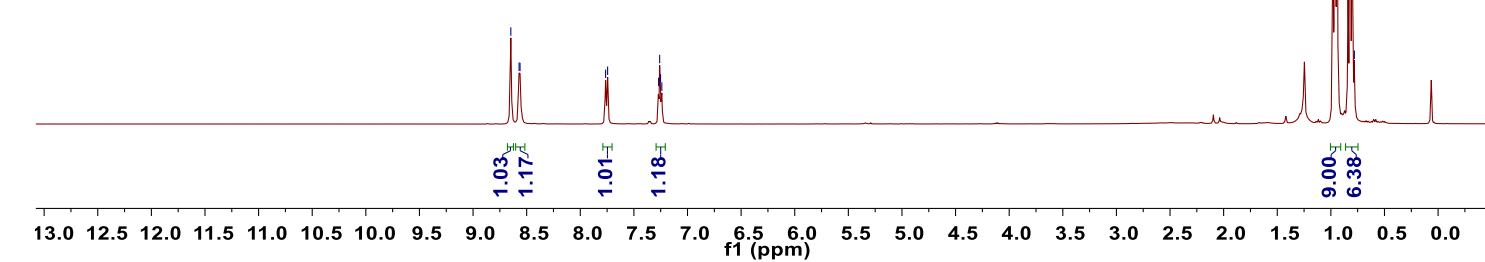

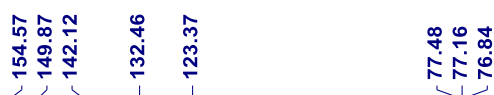

กิ๊

$\underbrace{\mathrm{SiEt}_{3}}_{N}$

${ }^{13} \mathrm{C}$ NMR, $100 \mathrm{MHz}, \mathrm{CDCl}_{3}$

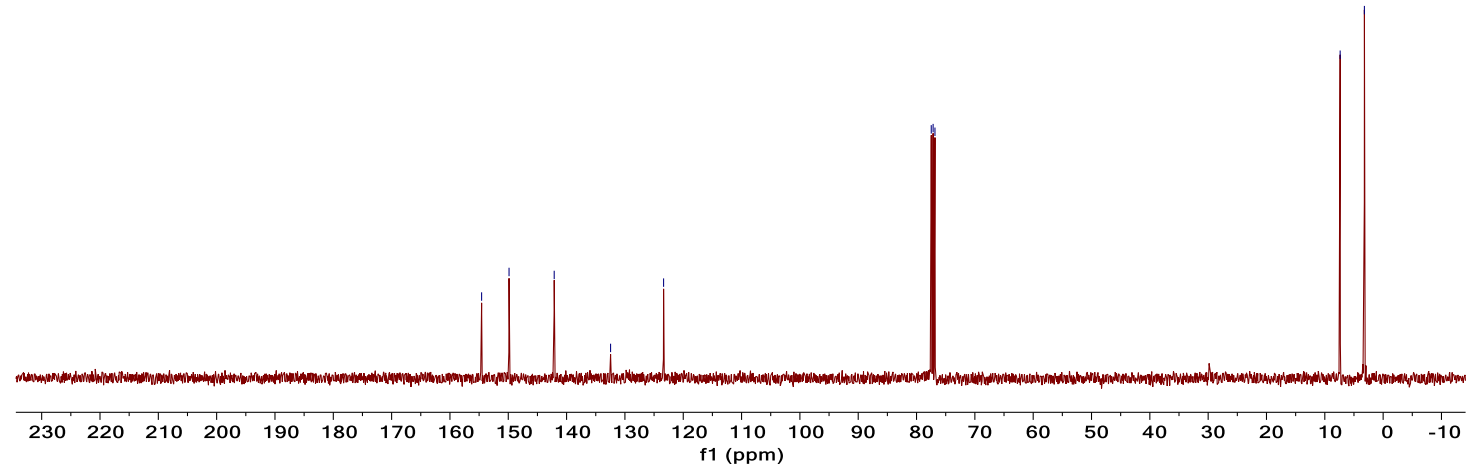


5-(triethylsilyl)-1H-indole (28).

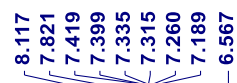

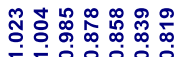

00

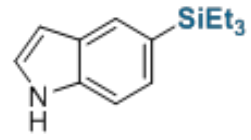

${ }^{1} \mathrm{H}$ NMR, 400MHz, $\mathrm{CDCl}_{3}$

H NMR, 400MHz, $\mathrm{CDCl}_{3}$

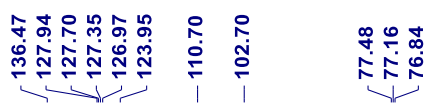

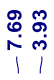

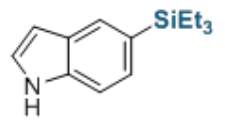

${ }^{13} \mathrm{C}$ NMR, $100 \mathrm{MHz}, \mathrm{CDCl}_{3}$

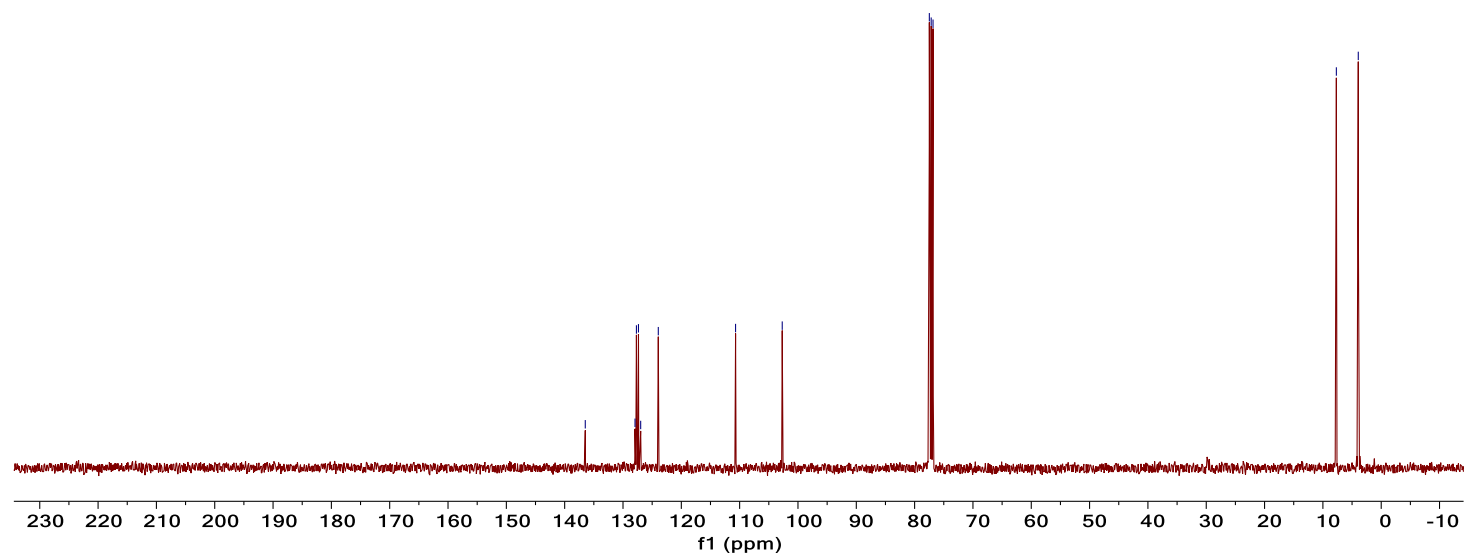


Tert-butyl 5-(triethylsilyl)-1H-indole-1-carboxylate (29).

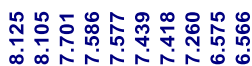

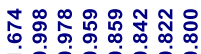

广००0000

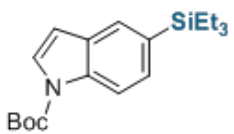

${ }^{1} \mathrm{H}$ NMR, $400 \mathrm{MHz}, \mathrm{CDCl}_{3}$

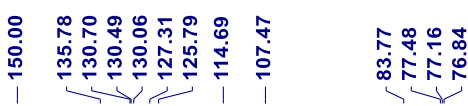

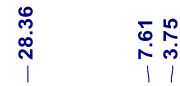

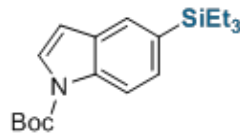

${ }^{13} \mathrm{C}$ NMR, $100 \mathrm{MHz}, \mathrm{CDCl}_{3}$

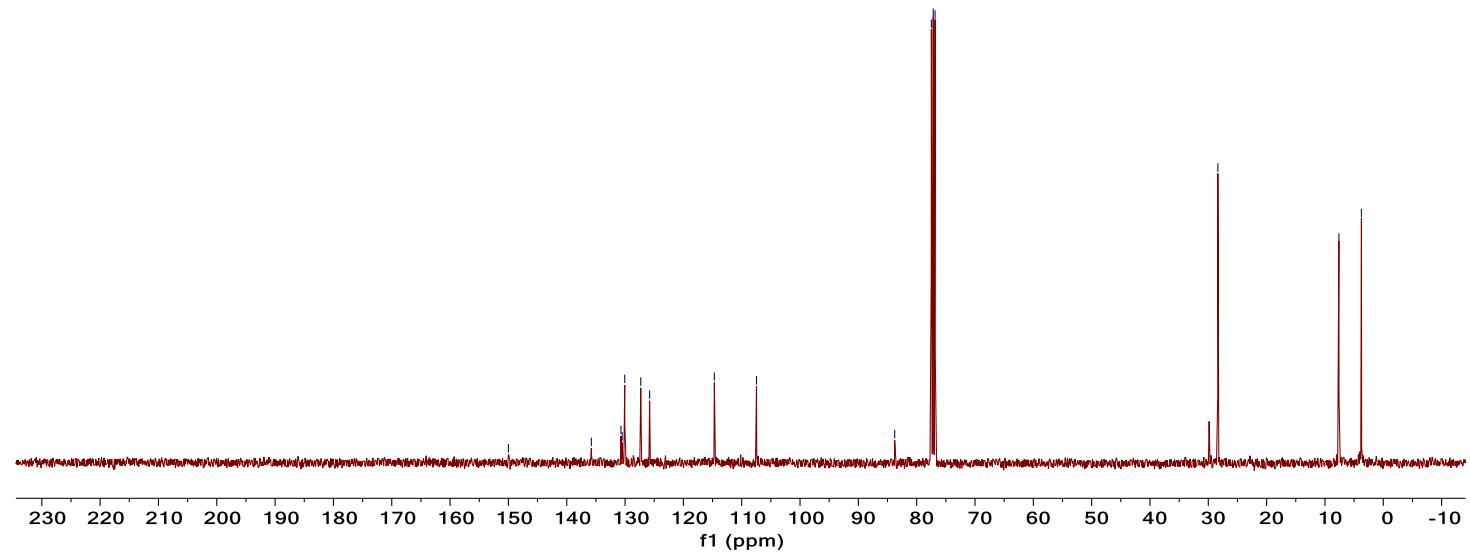


Tert-butyl-4-(phenyl(4-(triethylsilyl)phenyl)methyl)piperazine-1-carboxylate (30).

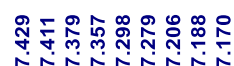

突

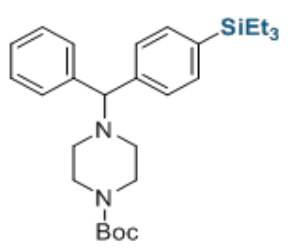

${ }^{1} \mathrm{H}$ NMR, $400 \mathrm{MHz}, \mathrm{CDCl}_{3}$

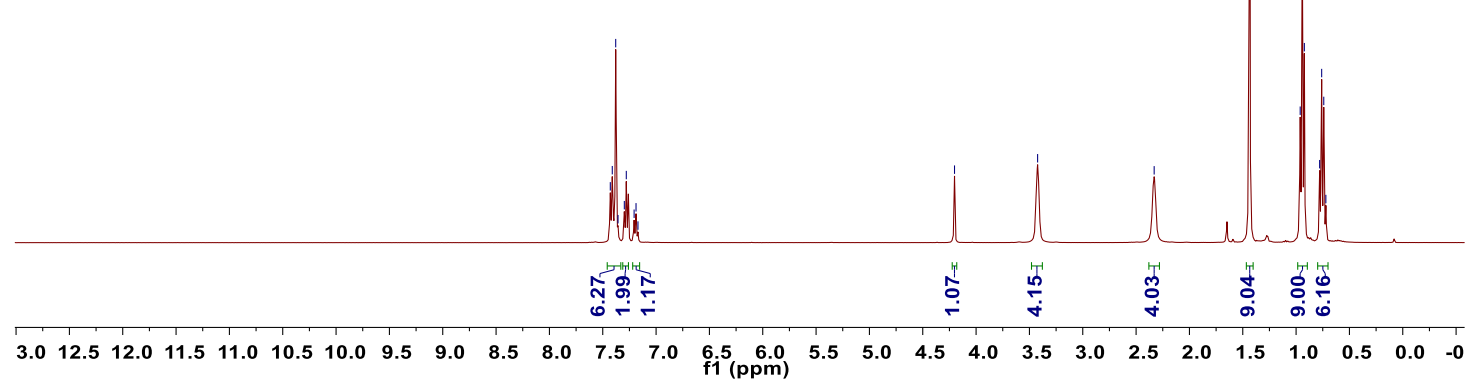

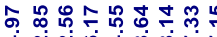

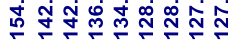

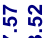

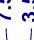

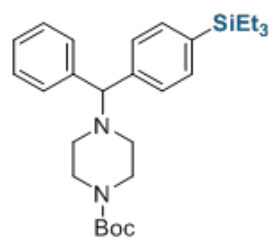

${ }^{13} \mathrm{C}$ NMR, $100 \mathrm{MHz}, \mathrm{CDCl}_{3}$

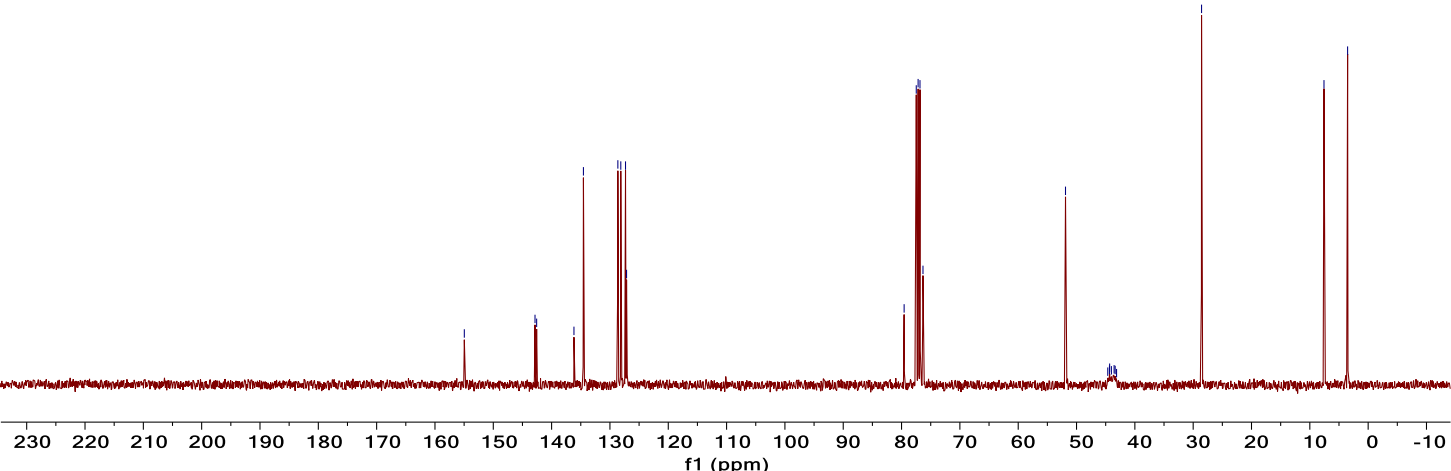


1-(2-(phenyl(4-(triethylsilyl)phenyl)methoxy)ethyl)piperidine (31).
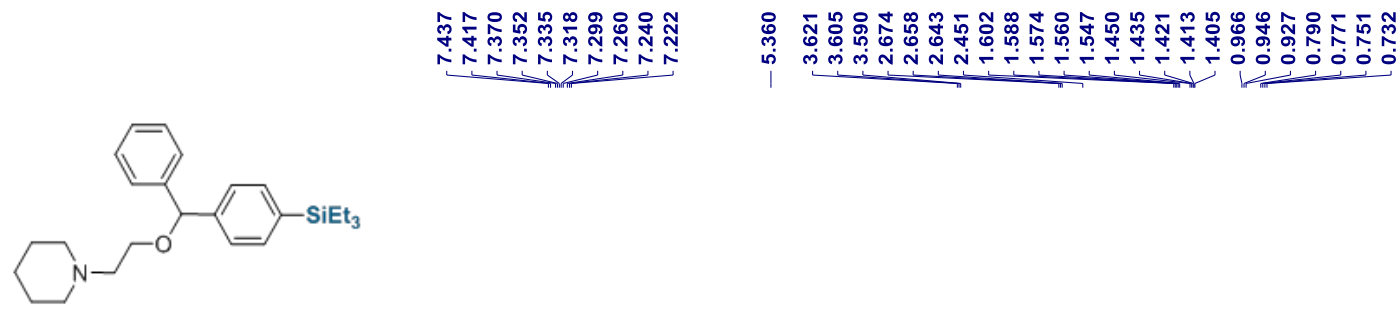

${ }^{1} \mathrm{H}$ NMR, $400 \mathrm{MHz}, \mathrm{CDCl}_{3}$

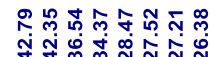

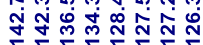

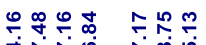

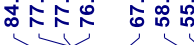

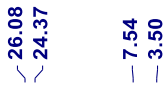

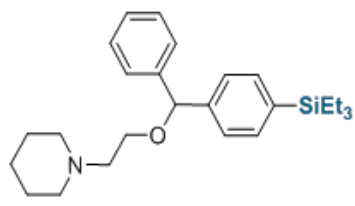

${ }^{13} \mathrm{C}$ NMR, $100 \mathrm{MHz}, \mathrm{CDCl}_{3}$

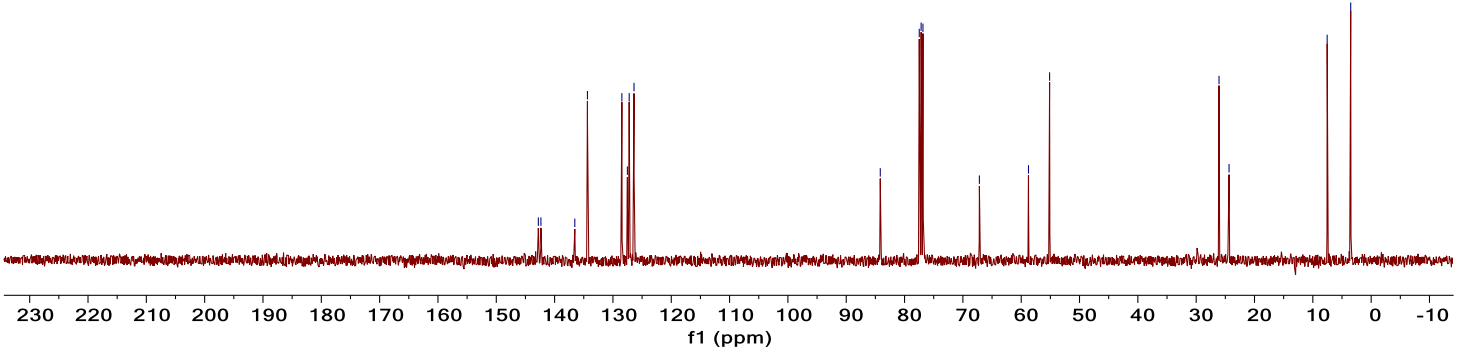


1-(4-(tert-butyl)benzyl)-4-(phenyl(4-(triethylsilyl)phenyl)methyl)piperazine (32).

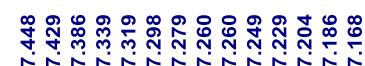

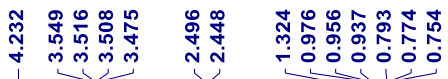

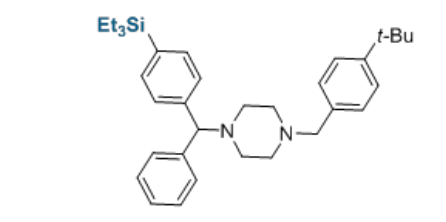

${ }^{1} \mathrm{H} \mathrm{NMR}, 400 \mathrm{MHz}, \mathrm{CDCl}_{3}$

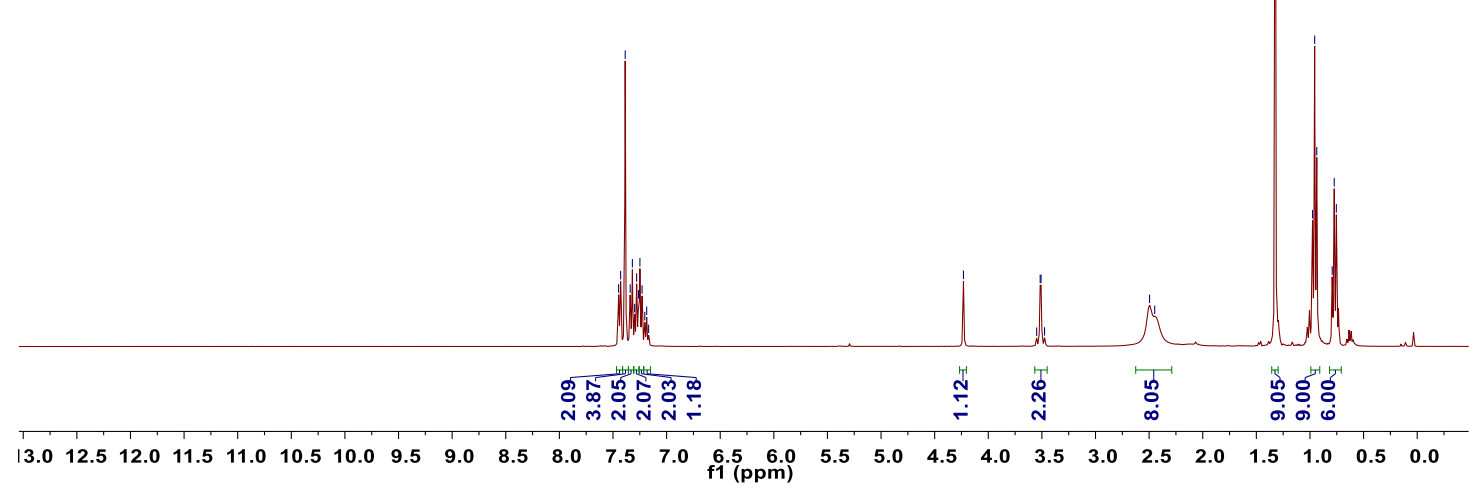

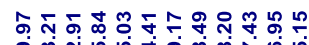

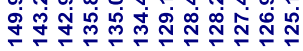

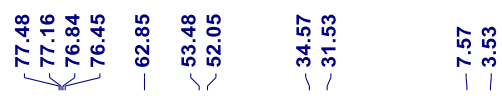

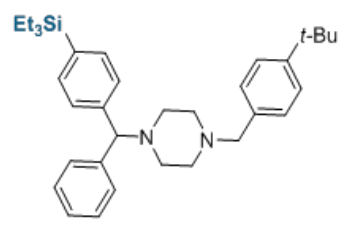

${ }^{13} \mathrm{C}$ NMR, 100MHz, $\mathrm{CDCl}_{3}$

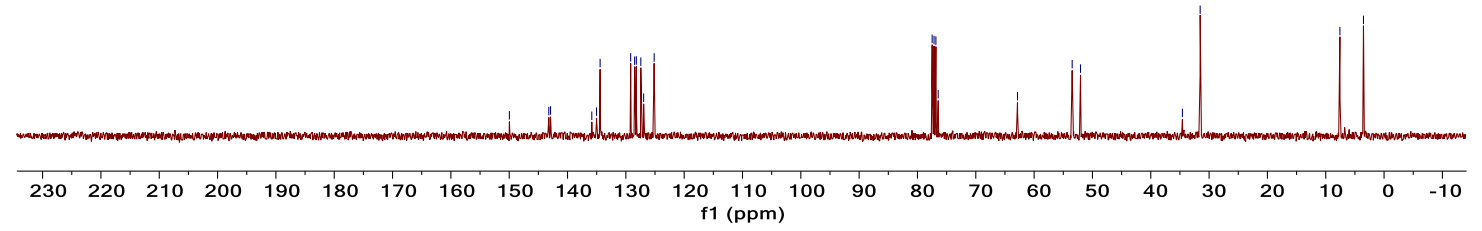


$\mathrm{N}, \mathrm{N}$-dimethyl-3-(3-(triethylsilyl)-10,11-dihydro-5H-dibenzo[b,f]azepin-5yl)propan-1-amine(33).

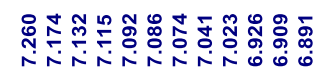

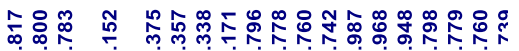

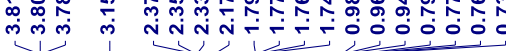

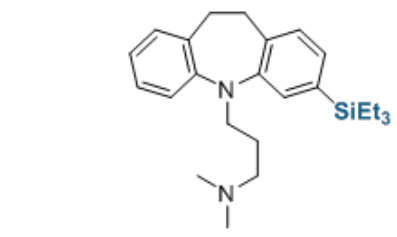

${ }^{1} \mathrm{H}$ NMR, 400MHz, $\mathrm{CDCl}_{3}$

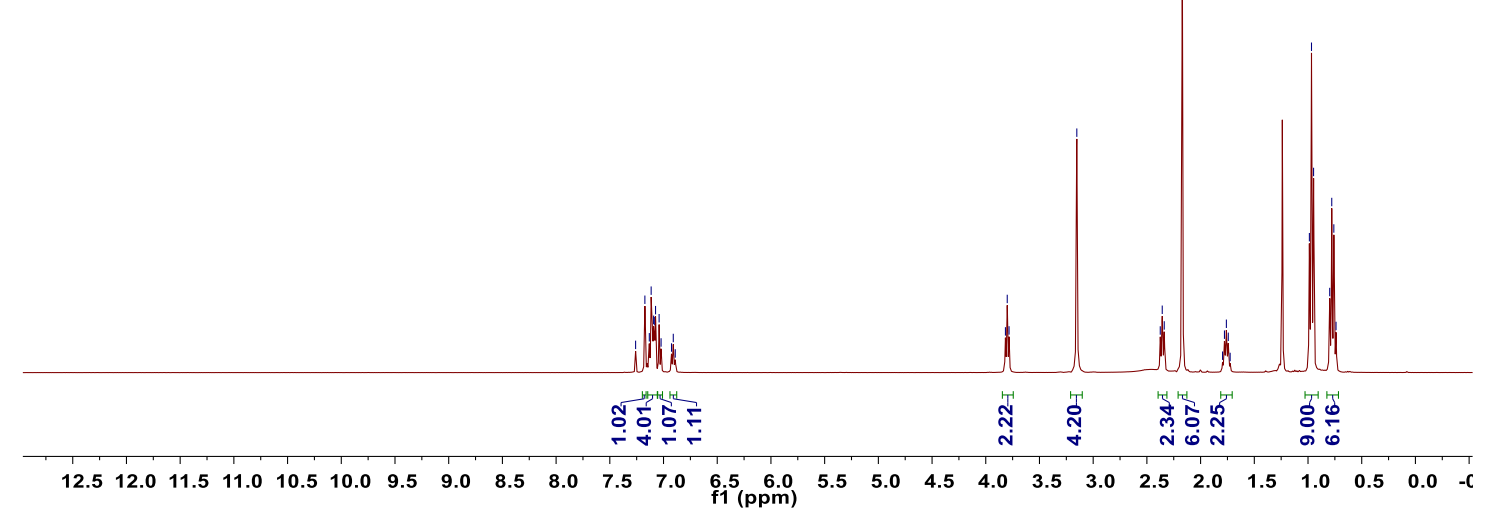

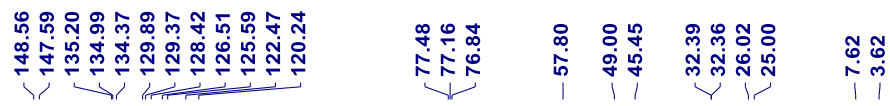

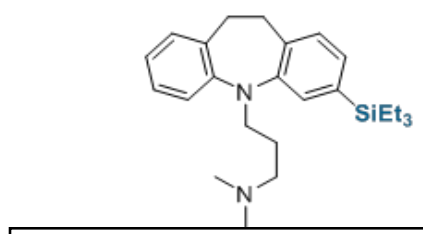

${ }^{13} \mathrm{C}$ NMR, 100MHz, $\mathrm{CDCl}_{3}$

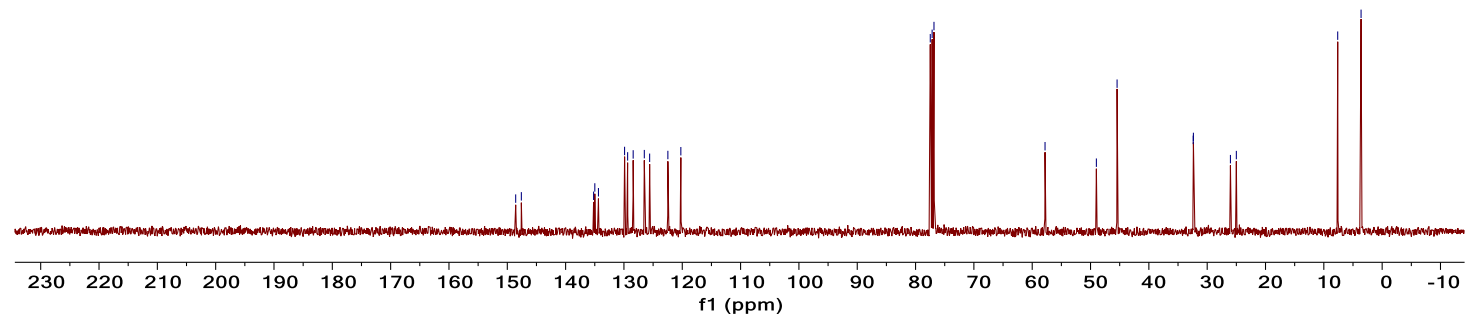




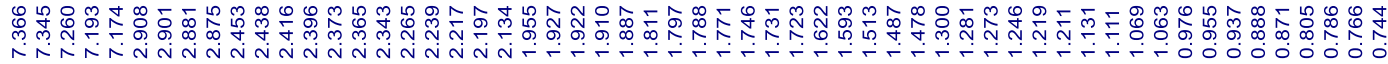
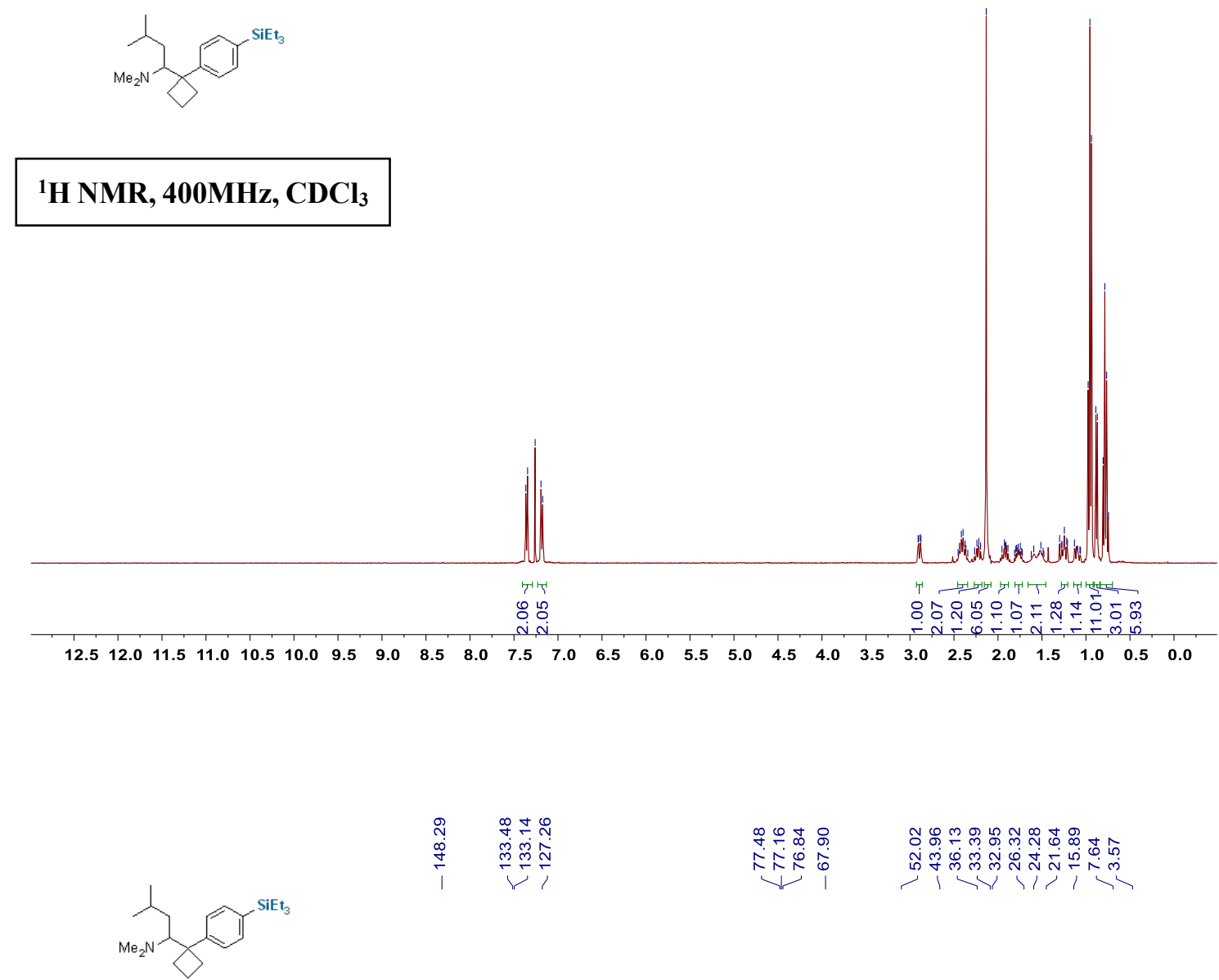

${ }^{13} \mathrm{C}$ NMR, $100 \mathrm{MHz}, \mathrm{CDCl}_{3}$ 